THE EXPONENTIAL REPRESENTATION OF FLOWS AND THE CHRONOLOGICAL CALCULUS

This article has been downloaded from IOPscience. Please scroll down to see the full text article. 1979 Math. USSR Sb. 35727

(http://iopscience.iop.org/0025-5734/35/6/A01)

The Table of Contents and more related content is available

Download details:

IP Address: 147.122.5.50

The article was downloaded on 25/06/2009 at 11:19

Please note that terms and conditions apply. 


\title{
THE EXPONENTIAL REPRESENTATION OF FLOWS AND THE CHRONOLOGICAL CALCULUS
}

UDC $517.938+517.317$

\author{
A. A. AGRAČEV AND R. V. GAMKRELIDZE
}

\begin{abstract}
In this article is developed a calculus which reflects the most general grouptheoretic properties of flows and which is based on an exponential representation of flows defined by nonstationary differential equations. Problems of optimization and control have had the greatest influence on the development of this calculus, and the results are intended mainly to treat these problems.

Bibliography: 11 titles.
\end{abstract}

This paper was written especially in honor of the seventieth birthday of Lev Semenovič Pontrjagin.

We develop here a calculus which is based on the exponential representation of flows defined by nonstationary ordinary differential equations and which reflects the most general group-theoretic properties of flows. The problems of the theory of optimization and control, and especially the numerous efforts to extend Pontrjagin's maximum principle to singular control problems (see [1]-[7]), have all had a decisive influence on our development of this calculus. In accordance with this basic concern, we treat those aspects of the calculus which have immediate applications to these theories.

As is well known, the main difficulty in expressing a flow by specifying its nonstationary vector field arises from the fact that the fields at different moments of time do not commute. This is a difficulty we must overcome in the derivations of all of the basic results in this paper, most of which have well-known analogs in the case of stationary flows. For similar "nonstationary" situations, physicists use the term "chronological," and we have adopted this term to describe the calculus developed here. It is explained briefly in [8].

We do not give a general overview of the content of the paper since it is sufficiently clear from the six section headings. We give here only the principal notation which will be used throughout.

We denote by $\mathbf{R}^{n} n$-dimensional real space, whose points are $n$-dimensional columns, always denoted by Latin letters; row vectors are always denoted by Greek letters. The scalar product of a row vector by a column vector of the same dimension will be written in the form of a matrix multiplication: 


$$
\xi \cdot x=\left(\xi_{1}, \ldots, \xi_{n}\right)\left(\begin{array}{c}
x^{1} \\
\vdots \\
x^{n}
\end{array}\right)=\sum_{\alpha=1}^{n} \xi_{\alpha} x^{\alpha}
$$

We denote the Jacobian matrix of an $m$-dimensional vector-valued function $x \mapsto X(x)$ with respect to the coordinate vector $x \in \mathbf{R}^{n}$ by

$$
\operatorname{grad} X=\left(\partial_{\beta} X^{\alpha}\right), \quad \alpha=1, \ldots, m, \beta=1, \ldots, n, \partial_{\beta}=\frac{\partial}{\partial x^{\beta}}, X=\left(\begin{array}{c}
X^{\mathbf{1}} \\
\vdots \\
X^{n}
\end{array}\right) .
$$

In particular, for a scalar function $\varphi$ we have

$$
\operatorname{grad} \varphi=\left(\partial_{1} \varphi, \ldots, \partial_{n} \varphi\right)
$$

The identity mapping of $\mathbf{R}^{n}$ onto itself will be denoted by $E$. By the modulus of a vector $x \in \mathbf{R}^{n}$ we understand the quantity $|x|=\max _{\alpha}\left|x^{\alpha}\right|$, and by the modulus of an $n$-dimensional row $\xi$, the quantity $|\xi|=\Sigma_{1}^{n}\left|\xi_{\alpha}\right|$. Similarly, we define the modulus of an $n \times m$ matrix $A=\left(a_{\beta}^{\alpha}\right), \alpha=1, \ldots, n, \beta=1, \ldots, m$, as $|A|=\sum_{\beta-1}^{m} \max _{\alpha}\left|a_{\beta}^{\alpha}\right|$. Finally, the term "smoothness" will mean infinite differentiability.

\section{\$1. Preparatory material}

We collect in this section the formulas and estimates which will be used in the subsequent development. In most cases they are well known, but we present them in some detail for the benefit of possible readers from the engineering specialties.

1. Differentiation and formal exponents in algebras. We consider an arbitrary real algebra $\mathcal{Q}$, i.e. a real vector space in which a multiplication of elements satisfying a unique bilinearity condition is defined. Thus $\mathbb{Q}$ is not necessarily associative and does not necessarily contain a unit; it can be a Lie algebra, for example, in which multiplication is anticommutative, $a b=-b a$, and the Jacobi identity

$$
a(b c)+b(c a)+c(a b)=0 \quad \forall a, b, c \in \mathbb{Q}
$$

is satisfied.

As is customary, we denote multiplication in an arbitrary Lie algebra by square brackets, $a b=[a, b]$, and we call it Lie brackets or commutation.

We denote the algebra of all linear mappings of the vector space $\mathbb{Q}$ into itself by $\mathcal{L}(\mathcal{Q})$, the product of the elements $\mathfrak{l}_{1}, \mathfrak{l}_{2} \in \mathcal{L}(\mathbb{Q})$, i.e., the composition of the linear mappings $\mathfrak{I}_{1}$ and $\mathfrak{I}_{2}$, by $\mathfrak{I}_{1} \circ \mathfrak{I}_{2}$, the identity element (identity mapping) by Id, and powers (iterations) of $\mathfrak{l}$ by $\mathfrak{l}^{m}, m>0$.

A linear mapping $\downarrow \in \mathcal{L}(\mathscr{Q})$ is called a differentiation in $\mathscr{Q}$ if it satisfies the formal rule for the differentiation of a product

$$
\delta(a b)=(\triangleright a) b+a(\triangleright b) .
$$

We denote the set of all differentiations in an algebra $\mathscr{Q}$ by $\operatorname{Der}(\mathbb{Q})$; it is a subspace, but not a subalgebra, of $\mathcal{L}(\mathbb{Q})$. However, we convert $\operatorname{Der}(\mathscr{Q})$ into a Lie algebra by defining the product $\left[\delta_{1}, \delta_{2}\right]$ of two elements $\delta_{1}, \delta_{2} \in \operatorname{Der}(\mathbb{Q})$ to be their commutator $\delta_{1} \circ \delta_{2}-$ $\delta_{2} \circ \delta_{1}$ :

$$
\left[\mathfrak{\delta}_{1}, \mathfrak{b}_{2}\right]=\mathfrak{\delta}_{1} \circ \delta_{2}-\mathfrak{\delta}_{2} \circ \delta_{1}
$$


The fact that the Lie brackets $\left[\delta_{1}, \delta_{2}\right]$ of two differentiations is again a differentiation in $\mathcal{Q}$, and also the Jacobi identity for the multiplication defined in this way can be verified by direct calculation.

Let $\Lambda$ be an arbitrary real Lie algebra. There exists a natural linear mapping ad: $\Lambda \rightarrow \mathcal{L}(\Lambda)$ given by

$$
(\operatorname{ad} v) w=[v, w] \quad \forall v, w \in \Lambda .
$$

We use the generally accepted notation

$$
\operatorname{ad}^{0} v=\mathrm{Id}, \quad \operatorname{ad}^{m+1} v=\operatorname{ad} v \circ \operatorname{ad}^{m} v=\operatorname{ad}^{m} v \circ \operatorname{ad} v
$$

for the powers of ad $v \in \mathcal{L}(\Lambda)$. The mapping ad has the following basic properties: it maps $\Lambda$ into $\operatorname{Der}(\Lambda)$,

$$
\operatorname{ad} v\left[w_{1}, w_{2}\right]=\left[\operatorname{ad} v w_{1}, w_{2}\right]+\left[w_{1}, \operatorname{ad} v w_{2}\right],
$$

where this mapping is a homomorphism of the Lie algebra $\Lambda$ into the Lie algebra $\operatorname{Der}(\Lambda)$, and

$$
\left(\operatorname{ad}\left[v_{1}, v_{2}\right]\right) \omega=\left[\operatorname{ad} v_{1}, \operatorname{ad} v_{2}\right] \omega .
$$

Both of these identities can be verified by direct calculation using the Jacobi identity.

In an arbitrary algebra $Q$ we may consider a formal power series over $\mathbb{Q}$ in the variable $t$,

$$
a_{t}=\sum_{\alpha=0}^{\infty} t^{\alpha} a_{\alpha}, \quad a_{\alpha} \in \mathbb{Q}
$$

We call the element $a_{0}$ the initial value of the series $a_{t}$. The set of all formal power series over $\mathbb{Q}$, which we denote by $\mathbb{Q}[[t]]$, can be made into a real algebra in a natural way if we add series and multiply a series by a number in the usual way and if we define the product of two series to be the "Cauchy product,"

$$
a_{t} b_{t}=\sum_{m=0}^{\infty} t^{m} \sum_{\alpha+\beta=m} a_{\alpha} b_{\beta} .
$$

The algebra $\mathbb{Q}$ is identified in an obvious canonical way with a subalgebra of $\mathbb{Q}[[t]]$.

We define the linear mapping $d / d t$ of the algebra $\mathcal{Q}[[t]]$ into itself, "differentiation with respect to $t$," by means of the usual "termwise differentiation,"

$$
\frac{d}{d t} a_{t}=\frac{d}{d t} \sum_{\alpha=0}^{\infty} t^{\alpha} a_{\alpha}=\sum_{\alpha=1}^{\infty} t^{\alpha-1}\left(\alpha a_{\alpha}\right)
$$

It is easy to verify by direct calculation that $d / d t \in \operatorname{Der}(\mathbb{Q}[[t]])$ and that

$$
\frac{d}{d t}\left(a_{t} b_{t}\right)=\left(\frac{d}{d t} a_{t}\right) b_{t}+a_{t}\left(\frac{d}{d t} b_{t}\right)
$$

We call the formal power series

$$
e^{t \mathfrak{l}}=\mathrm{Id}+t \mathfrak{l}+t^{2} \frac{1}{2 !} \mathfrak{l}^{2}+\ldots=\sum_{\alpha=0}^{\infty} t^{\alpha} \frac{\mathfrak{l}^{\alpha}}{\alpha !} \in \mathcal{L}(\mathbb{Q})[[t]]
$$

the formal exponential of the element $\mathfrak{l} \in \mathcal{L}(Q)$. 
The formal exponential $e^{t \mathfrak{l}}$ clearly commutes with $\mathfrak{l}$, $\mathfrak{l} e^{t \mathfrak{l}}=e^{t \mathfrak{l}} \mathfrak{l}$, and satisfies the "differential equation"

$$
\frac{d}{d t} e^{t \mathfrak{l}}=\mathfrak{l} e^{t \mathfrak{l}}
$$

It is easy to see that the equation

$$
\frac{d}{d t} a_{t}=\mathfrak{l} a_{t}, \quad \mathfrak{l} \in \mathcal{L}(\mathscr{Q}),
$$

in $a_{t} \in \mathbb{Q}[[t t]]$ with arbitrary initial value $a_{0} \in \mathbb{Q}$ has a unique solution $a_{t}$ given by $a_{t}=e^{t l} a_{0}$. This fact will be used below in the following form.

The equation

$$
\frac{d}{d t} \mathfrak{l}_{t}=\mathfrak{I}_{t}, \quad \mathfrak{l} \in \mathfrak{L}^{\prime}(\mathbb{Q}),
$$

in $\mathfrak{l}_{t} \in \mathcal{L}(\mathbb{Q})[[t]]$ with arbitrary initial value $\mathfrak{I}_{0}$ for $\mathfrak{I}$ has the unique solution $\mathfrak{I}_{t}=e^{t \mathfrak{I}_{0}}$. The validity of this statement becomes evident if we note that $\mathfrak{I}$ can be considered as a linear mapping of the vector space $\mathcal{L}(\mathbb{Q})$ into itself:

$$
\mathfrak{l}^{\prime} \mapsto \mathfrak{l} \circ \mathfrak{l}^{\prime} \quad \forall \mathfrak{l}^{\prime} \in \mathcal{L}(\mathbb{Q}) .
$$

For arbitrary elements $\mathfrak{l}_{1}, \mathfrak{l}_{2} \in \mathfrak{L}(\mathbb{Q})$ which commute, i.e. which satisfy

$$
\mathfrak{l}_{1} \circ \mathfrak{l}_{2}-\mathfrak{l}_{2} \circ \mathfrak{l}_{1}=\left[\mathfrak{l}_{1}, \mathfrak{l}_{2}\right]=0,
$$

we have that

$$
e^{t\left(I_{1}+l_{2}\right)}=e^{t I_{1}} e^{t I_{2}}=e^{t I_{2}} e^{t I_{1}}
$$

The formal exponential gives a bijective mapping $e^{t t}: a_{t} \mapsto e^{t l} a_{t}$ of $\mathbb{E}[[t]]$ onto itself, because $e^{t_{0}}=$ Id and therefore $e^{t \mathfrak{l}}=e^{t(-\mathfrak{l})}=\operatorname{Id} \forall \mathfrak{I} \in \mathcal{L}(\mathbb{Q})$.

If $\delta$ is a differentiation in $\mathcal{Q}$, then the formal exponential of $\mathfrak{d}$ has the following remarkable property.

For each differentiation $\delta \in \operatorname{Der}(\mathscr{Q})$ and for arbitrary $a, b \in \mathbb{Q}$ we have

$$
e^{t \mathrm{~b}}(a b)=\left(e^{t_{0}} a\right)\left(e^{t \mathrm{t}} b\right)
$$

In fact, since $d / d t$ and $\delta$ are differentiations in $\mathscr{Q}[[t]]$, we have the identity

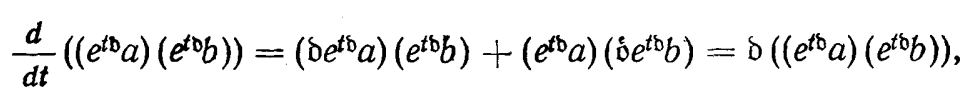

from which (1.2) follows if we take into consideration that the initial value of the formal power series $\left(e^{t s} a\right)\left(e^{t b} b\right)$ is $a b$.

Multiplying the power series on the right-hand side of (1.2) and equating the coefficients of equal powers of $t$ on both sides of the equation, we get the "Leibniz formula" for the $n$th power of a differentiation of a product, for a differentiation $\mathfrak{b} \in \operatorname{Der}(\mathbb{Q})$ in an arbitrary algebra $\mathbb{Q}$,

$$
\mathfrak{\delta}^{m}(a b)=\sum_{\alpha+\beta=m} \frac{m !}{\alpha ! \beta !}\left(\delta^{\alpha} a\right)\left(\delta^{\beta} b\right) .
$$


In concluding this subsection, we prove the "polarization identity" which we use in making estimates.

Let $\mathfrak{I}_{1}, \ldots, \mathfrak{I}_{m}$ be pairwise commuting elements of $\mathcal{L}(Q)$, i.e. $\left[\mathfrak{l}_{j}, \mathfrak{l}_{k}\right]=0$ for all $j, k=$ $1, \ldots, m$. Then

$$
\mathfrak{l}_{1} \circ \ldots \circ \mathfrak{l}_{m}=\frac{1}{m !} \sum_{\alpha=1}^{m}(-1)^{m-\alpha} \sum_{1=k_{1}<\ldots<k_{\alpha} \leqslant m}\left(\mathfrak{l}_{k_{1}}+\ldots \mathfrak{l}_{k_{\alpha}}\right)^{m} .
$$

Proof. Using the Viète formulas for expressing the coefficients of a polynomial as symmetric functions of its roots, and making use of (1.1), we can represent the formal power series

$$
\mathfrak{l}_{t}=\left(e^{t \mathfrak{l}_{1}}-\mathrm{Id}\right) \cdots\left(e^{t \mathfrak{l}_{m}}-\mathrm{Id}\right)=t^{m} \mathfrak{l}_{1} \circ \cdots \circ \mathfrak{I}_{m}+\ldots
$$

in the form

$$
\begin{aligned}
\mathfrak{l}_{t} & =(-\mathrm{Id})^{m}+\sum_{\alpha=1}^{m}(-1)^{m-\alpha} \sum_{1 \leqslant k_{1}<\ldots<k_{\alpha} \leqslant m} e^{t \mathfrak{l}_{k_{1}}} \ldots e^{t \mathfrak{l}_{k_{\alpha}}} \\
& =(-\mathrm{Id})^{m}+\sum_{\alpha=1}^{m}(-1)^{m-\alpha} \sum_{1 \leqslant k_{1}<\ldots<k_{\alpha} \leqslant m} e^{t\left(\mathfrak{r}_{k_{1}}+\ldots+\mathfrak{r}_{k_{\alpha}}\right)} .
\end{aligned}
$$

All of the coefficients of $t^{j}$ in the series on the right-hand side are equal to zero for $j<m-1$, and the coefficient of $t^{m}$ can be calculated directly to be

$$
\frac{1}{m !} \sum_{\alpha=1}^{m}(-1)^{m-\alpha} \sum_{1 \leqslant k_{1}<\ldots<k_{\alpha} \leqslant m}\left(\mathfrak{l}_{k_{1}}+\ldots+\mathfrak{l}_{k_{\alpha}}\right)^{m},
$$

which proves (1.4).

2. The algebras $\Phi$ and $\mathcal{L}(\Phi)$. We denote the algebra of all smooth real functions on $\mathbf{R}^{n}$ by $\Phi$, and the Cartesian product of $n$ copies of the vector space $\Phi=\Phi^{1}$ by $\Phi^{n}$. We identify canonically each linear mapping $\mathfrak{l}$ of $\Phi$ into itself with a linear mapping of $\Phi^{n}$ into itself in a canonical way by

$$
\mathfrak{l} X=\mathfrak{l}\left(\begin{array}{c}
X^{1} \\
\vdots \\
X^{n}
\end{array}\right)=\left(\begin{array}{c}
\mathfrak{l} X^{1} \\
\vdots \\
\mathfrak{l} X^{n}
\end{array}\right), \quad X=\left(\begin{array}{c}
X^{1} \\
\vdots \\
X^{n}
\end{array}\right) \in \Phi^{n}
$$

An arbitrary differentiation $\mathfrak{d} \in \operatorname{Der}(\Phi)$ in $\Phi$ is called a vector field, or simply a field over $\mathbf{R}^{n}$. As is well known, every vector field over $\mathbf{R}^{n}$ is a first-order differential operator, i.e, there exists a function

$$
X=\left(\begin{array}{c}
X^{1} \\
\vdots \\
X^{n}
\end{array}\right) \in \Phi^{n}
$$

such that

$$
\delta=\sum_{\alpha=1}^{n} X^{\alpha} \partial_{\alpha}, \quad \partial_{\alpha}=\frac{\partial}{\partial x^{\alpha}}
$$


Therefore in what follows we will denote vector fields over $\mathbf{R}^{n}$ by $\vec{X}, \vec{Y}$, etc., putting an arrow over the corresponding function. For constant fields, i.e. fields which correspond to constant functions, we use the notation $\vec{h}, h \in \mathbf{R}^{n}$. A field $\vec{X}$ and its corresponding function $X$ are obviously related by $\vec{X} E=X$.

As usual, we call the $m$-linear mapping $\mathbf{R}^{n} \times \cdots \times \mathbf{R}^{n} \rightarrow \mathbf{R}$ given by

$$
\left(h_{1}, \ldots, h_{m}\right) \mapsto \vec{h}_{1} \circ \ldots \circ \vec{h}_{m} \varphi(x)
$$

the $m$ th-order derivative of $\varphi \in \Phi$ at the point $x \in \mathbf{R}^{n}$. The symmetry of the mapping follows from the fact that constant fields commute, and the $m$-linearity is obvious. We get the usual expression for the first-order derivative,

$$
h \mapsto \vec{h} \varphi(x)=\operatorname{grad} \varphi(x) \cdot h=\sum_{\alpha=1}^{n} \partial_{\alpha} \varphi(x) h^{\alpha},
$$

and the formal Taylor series of a function $\varphi \in \Phi$ at a point $x$ can be written by making use of the formal exponential of $\vec{h}$ :

$$
\sum_{m=0}^{\infty} t^{m} \frac{\overrightarrow{h^{m}} \varphi}{m !}(x)=\left(\sum_{m=0}^{\infty} t^{m} \frac{\overrightarrow{h^{m}}}{m !}\right) \varphi(x)=e^{t \vec{h}} \varphi(x) .
$$

In this case, (1.2) and (1.3) take the form

$$
e^{\vec{h}}\left(\varphi_{1} \varphi_{2}\right)=\left(e^{t \vec{h}} \varphi_{1}\right)\left(e^{t \vec{h}} \varphi_{2}\right), \quad \vec{h}^{m}\left(\varphi_{1} \varphi_{2}\right)=\sum_{\alpha+\beta=m} \frac{m !}{\alpha ! \beta !} \vec{h}^{\alpha} \varphi_{1} \vec{h}^{\beta} \varphi_{2}
$$

For an arbitrary integer $s \geqslant 0$ and arbitrary $M \subset \mathbf{R}^{n}$, we consider the seminorm $\|\varphi\|_{s, M}$, which can be either finite or infinite, defined in $\Phi$ by

$$
\|\varphi\|_{s, M}=\sup _{x \in M} \sum_{a=0}^{s} \frac{1}{a !} \sup _{|h|=1}\left|\vec{h}^{\alpha} \varphi(x)\right| .
$$

If $M=\mathbf{R}^{n}$, we will omit the index $M$, i.e. $\|\varphi\|_{s, \mathbf{R}^{n}}=\|\varphi\|_{s}$. For matrix-valued functions, we let

$$
\|A\|_{s, M}=\sum_{\beta=1}^{n} \max _{\alpha}\left\|a_{\beta}^{\alpha}\right\|_{s, M}, \quad A=\left(a_{\beta}^{\alpha}\right), \quad a_{\beta}^{\alpha} \in \Phi
$$

in particular,

$$
\begin{gathered}
\|X\|_{s, M}=\max _{\alpha}\left\|X^{\alpha}\right\|_{s, M}, \quad X=\left(\begin{array}{c}
X^{1} \\
\vdots \\
X^{n}
\end{array}\right) \in \Phi^{n}, \\
\|\xi\|_{s, M}=\sum_{\beta=1}^{n}\left\|\xi_{\beta}\right\|_{s, M}, \quad \xi=\left(\xi_{1}, \ldots, \xi_{n}\right) .
\end{gathered}
$$

The following easily verified inequalities hold:

$$
\begin{gathered}
\|\varphi\|_{s, M} \leqslant\|\varphi\|_{s+k, M} \quad \forall k \geqslant 0, \\
\left\|\varphi_{1}\right\|_{s, M}-\left\|\varphi_{2}\right\|_{s, M} \mid \leqslant\left\|\varphi_{1}-\varphi_{2}\right\|_{s, M}, \\
\left\|\varphi_{1} \varphi_{2}\right\|_{s, M} \leqslant\left\|\varphi_{1}\right\|_{s, M}\left\|\varphi_{2}\right\|_{s, M},
\end{gathered}
$$


and their analogs for matrix-valued functions, of which we note

$$
\|A X\|_{s, M} \leqslant\|A\|_{s, M}\|X\|_{s, M} .
$$

We prove the inequality

$$
\sup _{x \in M,\left|h_{1}\right|=\ldots=; h_{s} \mid=1}\left|\vec{h}_{1} \circ \ldots \circ \vec{h}_{s} \varphi(x)\right| \leqslant(2 s)^{s}\|\varphi\|_{s, M} \quad \forall \varphi \in \Phi,
$$

which is important in making subsequent estimates. We use the polarization identity (1.4) and write

$$
\begin{aligned}
\sup _{x \in M,\left|h_{j}\right|=1}\left|\vec{h}_{1} \circ \ldots \circ \vec{h}_{s} \varphi(x)\right| \leqslant \frac{1}{s !} \sum_{\alpha=1}^{s} \sum_{k_{1}<\ldots<k_{\alpha}} \sup _{x \in M,\left|h_{k_{j}}\right|=1}\left|\left(\vec{h}_{k_{1}}+\ldots+\vec{h}_{k_{\alpha}}\right)^{s} \varphi(x)\right| \\
\leqslant \frac{1}{s !} \sum_{\alpha=1}^{s} \frac{s !}{\alpha !(s-\alpha) !} \sup _{x \in M,|b| \leqslant \alpha}\left|\vec{h}^{s} \varphi(x)\right| \\
\leqslant \frac{1}{s !} \sum_{\alpha=1}^{s} \alpha^{s} \frac{s !}{\alpha !(s-\alpha) !} \sup _{x \in M,|h|=1}\left|\vec{h}^{s} \varphi(x)\right| \\
\leqslant\|\varphi\|_{s, M^{s}} \sum_{\alpha=1}^{s} \frac{s !}{\alpha !(s-\alpha) !}=(2 s)^{s}\|\varphi\|_{s, M} .
\end{aligned}
$$

We introduce in $\Phi$ the topology defined by the family of seminorms $\|\cdot\|_{s, K}$, where $s>0$ and $K$ is an arbitrary compact set in $\mathbf{R}^{n}$. This topology, called the topology of compact convergence with respect to all derivatives, makes $\Phi$ a Fréchet space (a complete, metrizable, locally convex space), and we will always regard $\Phi$ as being furnished with this topology in what follows, unless otherwise specified.

We denote the algebra of continuous linear mappings of $\Phi$ into itself by $\mathcal{L}(\Phi)$. We show that $\mathscr{L}(\Phi)$ contains all vector fields. It is obviously sufficient to establish the inequality

$$
\|\vec{X} \varphi\|_{s, M} \leqslant 3 n(2 s+2)^{s+1}\|X\|_{s, M}\|\varphi\|_{s+\mathbf{1}, M},
$$

and its immediate consequences,

$$
\begin{aligned}
& \|\vec{X} A\|_{s, M} \leqslant 3 n(2 s+2)^{s+1}\|X\|_{s, M}\|A\|_{s+1, M}, \\
& \|\vec{X} Y\|_{s, M} \leqslant 3 n(2 s+2)^{s+1}\|X\|_{s, M}\|Y\|_{s+1, M}, \\
& \|\operatorname{grad} \varphi\|_{s, M} \leqslant 3 n(2 s+2)^{s+1}\|\varphi\|_{s+1, M} .
\end{aligned}
$$

Making use of (1.8), we can write

$$
\begin{aligned}
& \left\|\partial_{k} \varphi\right\|_{s, M} \leqslant \sum_{\alpha=0}^{s} \frac{1}{\alpha !} \sup _{x \in M,\left|h_{j}\right|=1}\left|\vec{h}_{1} \circ \ldots \circ \vec{h}_{\alpha+1} \varphi(x)\right| \\
& \leqslant \sum_{s=0}^{s} \frac{1}{\alpha !}(2 \alpha+2)^{\alpha+1}\|\varphi\|_{\alpha+1, M} \leqslant 3(2 s+2)^{s+1}\|\varphi\|_{s+1, M},
\end{aligned}
$$


whence

$$
\begin{aligned}
\|\vec{X} \varphi\|_{s, M}= & \left\|\sum_{\alpha=1}^{n}\left(\partial_{\alpha} \varphi\right) X^{\alpha}\right\|_{s, M} \leqslant \sum_{\alpha=1}^{n}\left\|\partial_{\alpha} \varphi\right\|_{s, M}\left\|X^{\alpha}\right\|_{s, M} \\
& \leqslant 3 n(2 s+2)^{s+1}\|X\|_{s, M}\|\varphi\|_{s+1, M^{*}}
\end{aligned}
$$

Above we put into correspondence with each function $X \in \Phi^{n}$ the field of $X$. Now we put into correspondence with each function $P \in \Phi^{n}$ another operator $\hat{P}$ in $\mathcal{L}(\Phi)$, which we call a smooth mapping; to distinguish it from the field generated by the same function $\boldsymbol{P}$, we will put a caret over the letter instead of an arrow. We define the action of $\hat{\boldsymbol{P}}$ on $\varphi \in \Phi$ by

$$
\hat{P}_{\varphi}=\varphi \circ P
$$

where the circle on the right-hand side denotes composition of functions. Thus,

$$
\begin{gathered}
\hat{P}\left(\lambda \varphi_{1}+\mu \varphi_{2}\right)=\lambda \hat{P} \varphi_{1}+\mu \hat{P}_{\varphi_{2}}, \quad\left(\hat{P}_{1}+\hat{P}_{2}\right) \varphi=\hat{P}_{1} \varphi+\hat{P}_{2} \varphi \\
\hat{P} E=E \circ P=P .
\end{gathered}
$$

To prove that $\hat{P}$ is continuous, we have the estimate

$$
\|\hat{P} \varphi\|_{s, M} \leqslant\left(1+9 n^{2}(2 s)^{2 s}\right)^{s}\left(1+\|P\|_{s, M}\right)^{s}\|\varphi\|_{s, P(M)}
$$

and its consequences,

$$
\begin{aligned}
& \|\hat{P} A\|_{s, M} \leqslant\left(1+9 n^{2}(2 s)^{2 s}\right)^{s}\left(1+\|P\|_{s, M}\right)^{s}\|A\|_{s, P(M)}, \\
& \|\hat{P} X\|_{s, M} \leqslant\left(1+9 n^{2}(2 s)^{2 s}\right)^{s}\left(1+\|P\|_{s, M}\right)^{s}\|X\|_{s, P(M)} .
\end{aligned}
$$

The proof is by induction on $s$. We have

$$
\begin{gathered}
\left\|\hat{P}_{\varphi}\right\|_{0, M}=\sup _{x \in M}|\varphi \circ P(x)|=\|\varphi\|_{0, P(M)}, \\
\|\varphi \circ P\|_{s, M} \leqslant\|\varphi \circ P\|_{s-1, M}+\frac{1}{s !} \sup _{x \in M,|h|=1}\left|\vec{h}^{s-1} \circ \vec{h} \varphi \circ P(x)\right| \\
\leqslant\|\varphi \circ P\|_{s-1, M}+\|(\operatorname{grad} \varphi) \circ P\|_{s-1, M}\|\vec{h} P\|_{s-1, M} .
\end{gathered}
$$

Furthermore, putting $C_{s}=\left(1+9 n^{2}(2 s)^{2 s}\right)^{s}$ and using the induction assumption and the second of the estimates (1.9), we can write

$$
\begin{aligned}
& \|(\operatorname{grad} \varphi) \circ P\|_{s-1, M}=\sum_{\alpha=1}^{n}\left\|\left(\partial_{\alpha} \varphi\right) \circ P\right\|_{s-1, M} \\
& \leqslant C_{s-1}\left(1+\|P\|_{s-1, M}\right)^{s-1} \sum_{\alpha=1}^{n}\left\|\partial_{\alpha} \varphi\right\|_{s-1, P(M)} \\
& \leqslant 3 n(2 s)^{s} C_{s-1}\left(1-\|P\|_{s-1, M}\right)^{s-1}\|\varphi\|_{s, P(M)},
\end{aligned}
$$


whence

$$
\begin{aligned}
& \|\varphi \circ P\|_{s, M} \leqslant\|\varphi \circ P\|_{s-1, M}+3 n(2 s)^{s} C_{s-1}\left(1+\|P\|_{s-1, M}\right)^{s-1}\|\overrightarrow{h P}\|_{s-1, M}\|\varphi\|_{s, P(M)} \\
& \quad \leqslant C_{s-1}\left(1+9 n^{2}(2 s)^{2 s}\left(1+\|P\|_{s, M}\right)^{s}\|\varphi\|_{s, P(M)} \leqslant C_{s}\left(1+\|P\|_{s, M}\right)^{s}\|\varphi\|_{s, P(M)} .\right.
\end{aligned}
$$

We prove one more estimate which we will need:

$$
\begin{gathered}
\left\|\left(\hat{P}_{1}-\hat{P}_{2}\right) X\right\|_{s, M} \leqslant 3 n(2 s+2)^{s+1}\left(1+9 n^{2}(2 s)^{2 s}\right)^{s}\left(1+\left\|P_{1}\right\|_{s, M}\right. \\
\left.\quad+\left\|P_{2}\right\|_{s, M}\right)^{s}\left\|P_{1}-P_{2}\right\|_{s, M}\|\varphi\|_{s+1}, \widetilde{M} \\
\widetilde{M}=\left\{y \mid y=P_{2}(x)+r\left(P_{1}(x)-P_{2}(x)\right), x \in M, 0 \leqslant r \leqslant 1\right\} .
\end{gathered}
$$

We have

$$
\begin{gathered}
\left(\hat{P}_{1}-\hat{P}_{2}\right) \varphi=\varphi \circ P_{1}-\varphi \circ P_{2}=\varphi \circ\left(P_{2}+\left(P_{1}-P_{2}\right)\right)-\varphi \circ P_{2} \\
=\int_{0}^{1} \frac{d}{d r} \varphi \circ\left(P_{2}+r\left(P_{1}-P_{2}\right)\right) d r=\int_{0}^{1}\left((\operatorname{grad} \varphi) \circ\left(P_{2}+r\left(P_{1}-P_{2}\right)\right)\right) \cdot\left(P_{1}-P_{2}\right) d r,
\end{gathered}
$$

and, on the basis of (1.10) and (1.9),

$$
\begin{gathered}
\left\|(\operatorname{grad} \varphi) \circ\left(P_{2}+r\left(P_{1}-P_{2}\right)\right)\right\|_{s, M} \\
\leqslant\left(1+9 n^{2}(2 s)^{2 s}\right)^{s}\left(1+\left\|P_{1}\right\|_{s, M}+\left\|P_{2}\right\|_{s, M}\right)^{s}\|\operatorname{grad} \varphi\|_{s, \widetilde{M}} \\
\leqslant 3 n(2 s+2)^{s+1}\left(1+9 n^{2}(2 s)^{2 s}\right)^{s}\left(1+\left\|P_{1}\right\|_{s, M}+\left\|P_{2}\right\|_{s, M}\right)^{s}\|\varphi\|_{s+1}, \widetilde{M}
\end{gathered}
$$

whence the desired estimate follows.

In what follows, all of the smooth mappings $P$ will, as a rule, be diffeomorphisms of $\mathbf{R}^{n}$. We will also call the corresponding operators $\hat{P} \in \mathcal{L}\left(\Phi^{n}\right)$ diffeomorphisms.

It is easy to see that if the operator $\mathfrak{l} \in \mathcal{L}(\Phi)$ satisfies

$$
\mathfrak{l} \varphi=\varphi \circ \mathfrak{l} E \quad \forall \varphi \in \Phi
$$

and is invertible in $\mathfrak{L}(\Phi)$, then $\mathfrak{l} E$ is a diffeomorphism of $\mathbf{R}^{n}$. Indeed, for all $\varphi \in \Phi$,

$$
\varphi=\varphi \circ\left(\mathfrak{l} \circ \mathfrak{l}^{-1} E\right)=\varphi \circ\left(\mathfrak{l}^{-1} E \circ \mathfrak{l} E\right)=\left(\varphi \circ \mathfrak{l}^{-1} E\right) \circ \mathfrak{l} E=\mathfrak{l}\left(\varphi \circ \mathfrak{l}^{-1} E\right),
$$

and consequently $\mathfrak{l}^{-1} \varphi=\varphi \circ \mathfrak{l}^{-1} E$. Therefore the smooth mappings $P=\mathfrak{l} E$ and $Q=$ $\mathfrak{l}^{-1} E$ are inverses of each other since

$$
P \circ Q=\mathfrak{l} E \circ \mathfrak{l}^{-1} E=\mathfrak{l}^{-1} \circ \mathfrak{l} E=E, \quad Q \circ P=\mathfrak{l}^{-1} E \circ \mathfrak{l} E=\mathfrak{l} \circ \mathfrak{l}^{-1} E=E .
$$

Conversely, if $P$ and $Q$ are smooth diffeomorphisms of $\mathbf{R}^{n}$ which are inverses of each other, then

$$
(\hat{P})^{-1}=\hat{Q} \text {. }
$$

We also note the obvious fact that if $P_{1}$ and $P_{2}$ are two smooth mappings of $\mathbf{R}^{n}$ into itself, and if $Q=P_{1} \circ P_{2}$ is their composition, then

$$
\hat{Q}=\hat{P}_{2} \circ \hat{P}_{1} \text {. }
$$

If $\hat{P}$ is a diffeomorphism, we can verify by direct calculation that for any field $\vec{X} \in \operatorname{Der}(\Phi)$ the composition $\hat{P} \circ \vec{X} \circ \hat{P}^{-1}$ is also a field. We consider the function $\vec{X} \mapsto \hat{P} \circ \vec{X} \circ \hat{P}^{-1} \in \operatorname{Der}(\Phi)$ as a linear mapping of $\operatorname{Der}(\Phi)$ into itself defined by the 
diffeomorphism $P$, and we denote it by Ad $P$ :

$$
(\text { Ad } \hat{P}) \vec{X} \stackrel{\text { def }}{=} \hat{P} \circ \vec{X} \circ \hat{P}^{-1} \in \operatorname{Der}(\Phi) \quad \forall \vec{X}
$$

3. Families of functions and operators. In what follows, we will constantly be concerned with families $\varphi_{t}, t \in \mathbf{R}$, of elements of $\Phi$, to which the basic constructions of analysis carry over in a natural way if we use the topology in $\Phi$. We note here only the most essential ones.

It is not necessary to give special definitions of continuity and differentiability with respect to $t$ of a family $\varphi_{t}$, since $\Phi$ is a topological vector space. We will say that a family $\varphi_{t}, t \in \mathbf{R}$, is measurable if $\forall x \in \mathbf{R}^{n}$ the scalar function $t \mapsto \varphi_{t}(x)$ is measurable, and a measurable family is locally integrable or uniformly integrable, respectively, if, for any given $t_{1}, t_{2}, s \geqslant 0$ and for any compact set $K \subset \mathbf{R}^{n}$,

$$
\int_{t_{1}}^{t_{2}}\left\|\varphi_{\tau}\right\|_{s, K} d \tau<\infty
$$

or

$$
\int_{t_{1}}^{t_{2}}\left\|\varphi_{\tau}\right\|_{s, K} d \tau \rightarrow 0 \quad \text { as } \quad\left|t_{1}-t_{2}\right| \rightarrow 0
$$

respectively. (The measurability of $\left\|\varphi_{t}\right\|_{s, K}$ follows easily from the measurability of $\varphi_{t}$.)

We call the function

$$
x \rightarrow \int_{t_{1}}^{t_{2}} \varphi_{\tau}(x) d \tau, \quad x \in \mathbf{R}^{n}
$$

the integral of the locally integrable family $\varphi_{t}, t \in \mathbf{R}$, between the limits $t_{1}$ and $t_{2}$. We prove by induction on $s$ that it belongs to $\Phi$ and that, for arbitrary constant fields $\vec{h}_{1}, \ldots, \vec{h}_{s}$

$$
\vec{h}_{1} \circ \ldots \circ \vec{h}_{s} \int_{t_{1}}^{t_{2}} \varphi_{\tau} d \tau=\int_{t_{1}}^{t_{2}} \vec{h}_{1} \circ \ldots \circ \vec{h}_{s} \varphi_{\tau} d \tau .
$$

Let $K$ be a compact neighborhood of an arbitrarily given point $x_{0} \in \mathbf{R}^{n}$. From (1.8) we have that

$$
\left|\vec{h}_{1} \circ \ldots \circ \vec{h}_{s} \varphi_{t}(x)\right| \leqslant(2 s)^{s}\left\|\varphi_{t}\right\|_{s, K} \quad \forall x \in K
$$

where the function on the right is integrable over $\left[t_{1}, t_{2}\right]$ by hypothesis. Applying the induction assumption and the theorem concerning differentiation under the integral sign at $x_{0}$, we get

$$
\vec{h}_{1}\left(\vec{h}_{2} \circ \ldots \circ \vec{h}_{\mathrm{s}} \int_{t_{1}}^{t_{2}} \varphi_{\tau} d \tau\right)=\vec{h}_{1} \int_{t_{1}}^{t_{2}} \vec{h}_{2} \circ \ldots \circ \vec{h}_{\mathrm{s}} \varphi_{\tau} d \tau=\int_{t_{1}}^{t_{2}} \vec{h}_{1} \circ \ldots \circ \vec{h}_{\mathrm{s}} \varphi_{\tau} d \tau
$$

The statement just proved implies directly that

$$
\left\|\int_{t_{1}}^{t_{2}} \varphi_{\tau} d \tau\right\|_{s, K} \leqslant \int_{t_{1}}^{t_{3}}\left\|\varphi_{\tau}\right\|_{s, K} d \tau
$$

from which it follows that $\int_{t_{1}}^{t_{2}} \varphi_{\tau} d \tau$ depends continuously on $\left(t_{1}, t_{2}\right)$. 
We call the family $\varphi_{t}, t \in \mathbf{R}$, absolutely continuous if there exists a locally integrable family $\psi_{t}$ such that $\varphi_{t}=\varphi_{t_{0}}+\int_{t_{0}}^{t} \psi_{\tau} d \tau$. Using the fact that there exists a countable set which is everywhere dense in $\Phi$, we can prove, just as for scalar functions, that for almost all $t$

$$
\frac{d}{d t} \varphi_{t}=\frac{d}{d t} \int_{t_{0}}^{t} \psi_{\tau} d \tau=\psi_{t}
$$

We now pass to a consideration of families of operators $\mathfrak{l}_{t}, t \in \mathbf{R}$, in $\mathcal{E}(\Phi)$, where all of the concepts of analysis defined above for families $\varphi_{t}$ carry over automatically to families of operators if the corresponding concept is defined for $\mathfrak{l}_{t}$ in the "weak" sense. For the sake of convenience in the formulation, we introduce the vector topology of simple (pointwise) convergence in $\mathcal{E}(\Phi)$ : a sequence of operators $\mathfrak{l}_{m}, m=1,2, \ldots$, converges to zero if and only if $\mathfrak{I}_{m} \varphi \rightarrow 0$ as $m \rightarrow \infty$ for all $\varphi \in \Phi$.

We define measurability, continuity, differentiability, local integrability, uniform integrability, and absolute continuity of the family $\mathfrak{l}_{t}, t \in \mathbf{R}$, by requiring that the family $\mathfrak{l}_{t} \varphi$ have the corresponding property for all $\varphi \in \Phi$.

We define the derivative of a family $\mathfrak{l}_{t}$ which is differentiable at $t_{0}$ to be the linear operator

$$
\mathfrak{l}_{t_{0}}^{\prime}=\lim _{\delta t_{m \rightarrow 0}} \frac{\mathfrak{l}_{t_{0}+\delta t_{m}}-\mathfrak{l}_{t_{0}}}{\delta t_{m}},
$$

the convergence here, as in all that follows, being in the topology of simple convergence in $\mathcal{E}(\Phi)$. By the Banach-Steinhaus theorem, the limit of a sequence of continuous linear operators in a Fréchet space is a continuous operator; consequently $\mathfrak{I}_{t_{0}}^{\prime} \in \mathcal{L}(\Phi)$.

The integral of a locally summable family $\mathfrak{l}_{t}$ from $t_{1}$ to $t_{2}$ is defined to be the linear operator which acts thus:

$$
\int_{t_{1}}^{t_{2}} \mathfrak{l}_{\tau} d \tau \varphi=\int_{t_{1}}^{t_{2}} \mathfrak{l}_{\tau} \varphi d \tau .
$$

We prove that this operator is continuous.

We denote by $\mathfrak{l}_{t}^{(m)}, t_{1} \leqslant t \leqslant t_{2}$, the "truncation" of a family $\mathfrak{l}_{t}$, defined as follows for each $m=1,2 \ldots$ We take a sequence of numbers $C_{m} \rightarrow \infty$ and an increasing sequence of compact sets $K_{m}$ whose union is all of $\mathbf{R}^{n}$. We fix $s$ and $K$ and put

$$
\mathfrak{l}_{t}^{(m)}=\mathfrak{l}_{t}, \quad \text { if } \quad\left\|\mathfrak{l}_{t} \varphi\right\|_{s, K} \leqslant C_{m}\|\varphi\|_{s+m, K_{m}} \quad \forall \varphi \in \Phi
$$

and $\mathfrak{I}_{t}^{(m)}=0$ otherwise. We denote by $\mathcal{E}_{m}$ the set of all points of $\left[t_{1}, t_{2}\right]$ at which $\mathfrak{I}_{t}^{(m)}=\mathfrak{l}_{t}$; obviously mes $\mathcal{E}_{m} \rightarrow t_{2}-t_{1}$ as $m \rightarrow \infty$. Finally, we denote by $\Phi_{s, K}$ the vector space $\Phi$ furnished with the topology generated by the single seminorm $\|\cdot\|_{s, K}$, and by $\mathcal{L}\left(\Phi, \Phi_{s, K}\right)$ the vector space of continuous linear mappings from $\Phi$ into $\Phi_{s, K}$. For each fixed $m$, the family $\mathfrak{I}_{t}^{(m)}, t_{1} \leqslant t \leqslant t_{2}$, is bounded in the topology of simple convergence, i.e., for any $\varphi, s$, and $K$ we can find a constant $C(\varphi, s, K)$ such that

$$
\left\|\mathfrak{r}_{t}^{(m)} \varphi\right\|_{s, K} \leqslant C(\varphi, s, K) \quad \forall t \in\left[t_{1}, t_{2}\right]
$$


Therefore by the Banach-Steinhaus theorem it is equicontinuous, and consequently $\int_{t_{1}}^{t_{2}} \mathfrak{l}_{\tau}^{(m)} d \tau \in \mathcal{L}\left(\Phi, \Phi_{s, K}\right)$, whence (again by the Banach-Steinhaus theorem)

$$
\lim _{m \rightarrow \infty} \int_{t_{1}}^{t_{3}} \mathfrak{l}_{\tau}^{(m)} d \tau=\lim _{m \rightarrow \infty} \int_{\mathbb{E}_{m}} \mathfrak{l}_{\tau} d \tau=\int_{t_{1}}^{t_{2}} \mathfrak{l}_{\tau} d \tau \in \mathcal{L}\left(\Phi, \Phi_{s, K}\right) .
$$

Since $s$ and $K$ are arbitrary here, $\int_{t_{1}}^{t_{2}} \mathfrak{l}_{\tau} d \tau \in \mathcal{E}(\Phi)$.

We call the family $\mathfrak{l}_{t}, t \in \mathbf{R}$, absolutely continuous if there exists a locally integrable family $\tilde{\mathfrak{I}}_{t}$ such that $\mathfrak{l}_{t}=\mathfrak{l}_{t_{0}}+\int_{t_{0}}^{t} \tilde{\mathfrak{I}}_{\tau} d \tau$. From the analogous statement for the family $\varphi_{t}$ it follows at once that $d \mathfrak{l}_{t} / d t=\tilde{\mathfrak{I}}_{t}$ for almost all $t$.

It is easy to see that if a family $m_{t}$ is locally integrable and the family $\mathfrak{l}_{t}$ is continuous, then $\mathfrak{I}_{t} \circ \mathfrak{m}_{t}$ is locally integrable.

In conclusion, we prove that if $\mathfrak{l}_{t}$ and $\mathrm{m}_{t}$ are continuous families of operators in $\mathcal{L}(\Phi)$ which are differentiable at $t_{0}$, then $\left(\mathfrak{l}_{t_{1}} \circ \mathfrak{m}_{t_{2}}\right)$ depends continuously on $\left(t_{1}, t_{2}\right) \in \mathbf{R}^{2}$, the family $\mathfrak{l}_{t} \circ \mathfrak{m}_{t}$ is differentiable at $t_{0}$, and we have the Leibniz formula for the derivative:

$$
\frac{d}{d t}\left(\mathfrak{l}_{t} \circ \mathfrak{m}_{t}\right)_{t_{0}}=\left(\frac{d}{d t} \mathfrak{l}_{t}\right)_{t_{0}} \circ \mathfrak{m}_{t_{0}}+\mathfrak{l}_{t_{0}} \circ\left(\frac{d}{d t} \mathfrak{m}_{t}\right)_{t_{0}} .
$$

To prove the continuity, we form the difference

$$
\left(\mathfrak{l}_{t_{1}+\delta t_{1}} \circ \mathfrak{m}_{t_{2}+\delta t_{2}}-\mathfrak{l}_{t_{1}} \circ \mathfrak{m}_{t_{2}}\right) \varphi=\mathfrak{l}_{t_{1}+\delta t_{1}} a\left(\mathfrak{m}_{t_{2}+\delta t_{2}}-\mathfrak{m}_{t_{2}}\right) \varphi+\left(\mathfrak{l}_{t_{1}+\delta t_{1}}-\mathfrak{l}_{t_{1}}\right) \circ \mathfrak{m}_{t_{2}} \varphi .
$$

As $\delta t_{1}, \delta t_{2} \rightarrow 0$, the second term $\left(\mathfrak{l}_{t_{1}+\delta t_{1}}-\mathfrak{l}_{t_{1}}\right) \circ \mathfrak{m}_{t_{2}} \varphi \rightarrow 0$ by definition, and $\left(\mathfrak{m}_{t_{2}}-\delta t_{2}-\mathfrak{m}_{t_{2}}\right) \varphi$ lies in any preassigned neighborhood of zero in $\Phi$. Furthermore the family $\mathfrak{l}_{t_{1}+\delta t_{1}},\left|\delta t_{1}\right| \leqslant$ const, is continuous and therefore bounded in the topology of simple convergence; consequently it is equicontinuous by the Banach-Steinhaus theorem, and therefore

$$
\mathfrak{l}_{t_{2}+\delta t_{1}} \circ\left(\mathfrak{m}_{t_{2}+\delta t_{2}}-\mathfrak{m}_{t_{2}}\right) \varphi \rightarrow 0 \text { as } \delta t_{1}, \delta t_{2} \rightarrow 0 .
$$

The statement concerning differentiability follows from identical arguments if we write

$$
\frac{1}{\delta t}\left(\mathfrak{l}_{t+\delta t} \circ \mathfrak{m}_{t+\delta t}-\mathfrak{l}_{t} \circ \mathfrak{m}_{t}\right)=\frac{1}{\delta t}\left(\mathfrak{l}_{t+\delta t}-\mathfrak{l}_{t}\right) \circ \mathfrak{m}_{t}+\frac{1}{\delta t} \mathfrak{l}_{t+\delta t} \sigma\left(\mathfrak{m}_{t+\delta t}-\mathfrak{m}_{t}\right)
$$

From what we have proved it follows easily that the composition $\mathfrak{l}_{t} \circ \mathfrak{m}_{t}$ of two absolutely continuous families is absolutely continuous, and also that the family $\mathfrak{l}_{t} \varphi_{t}$ is absolutely continuous if $\varphi_{t}$ is absolutely continuous, and that the formula for the differentiation of a product holds for it.

4. Nonstationary fields and flows. The main objects of our study are two classes of families of operators in $\mathcal{L}(\Phi)$. They are nonstationary fields and flows.

We will call an arbitrary locally integrable family $\vec{X}_{t}, t \in \mathbf{R}$, of vector fields over $\mathbf{R}^{n}$ a nonstationary field over $\mathbf{R}^{n}$, or simply a field. Each additional property of a field will be stated explicitly.

We will call an arbitrary absolutely continuous family of diffeomorphisms $\hat{P}_{t}$ which are defined for values of $t$ in a given interval $J$ a flow over $\mathbf{R}^{n}$. The interval $J$ can be replaced by the entire axis $\mathbf{R}$ in this definition (see Proposition 1.2); we prefer, however, for purely technical reasons to leave it unspecified.

We also call the family of diffeomorphisms $P_{t}=\hat{P}_{t} E$ of $\mathbf{R}^{n}$ corresponding to the flow $\hat{P}_{t}$ a flow. It is easy to see that $P_{t}$, together with all of its derivatives with respect to $x$, is continuous with respect to the collection of variables $(t, x)$ and is absolutely continuous with respect to $t$ for fixed $x \in \mathbf{R}^{n}$, and that it satisfies 


$$
\int_{t_{1}}^{t_{2}}\left\|\frac{d}{d \tau} P_{\tau}\right\|_{s, K} d \tau<\infty .
$$

Conversely, every family of smooth diffeomorphisms $P_{t}$ of $\mathbf{R}^{n}$ with these properties generates a flow $\hat{P}_{t}$ in the above sense.

We consider the linear differential equation

$$
\frac{d}{d t} \mathfrak{F}_{t}=\mathfrak{F}_{t} \circ \vec{X}_{t}
$$

with the initial conditions

$$
\mathfrak{F t}_{t_{0}}=\mathfrak{F}_{0}
$$

for the unknown family $\mathfrak{F}_{t}, t \in J_{t_{0}}$, of operators in $\mathcal{L}(\Phi)$. Here $\vec{X}_{t}$ is a given nonstationary field, and $J_{t_{0}}$ is an interval containing $t_{0}$. Every absolutely continuous family $\mathfrak{F}_{t}, t \in J_{t_{0}}$, of operators in $\mathcal{E}(\Phi)$ which satisfies (1.13) for almost all $t \in J_{t_{0}}$ and which satisfies (1.14) will be called a solution of (1.13) under the conditions (1.14). The absolute continuity of the unknown solution guarantees the equivalence of (1.13)-(1.14) and the integral equation

$$
\mathfrak{F}_{t}=\mathfrak{F}_{0}+\int_{t_{0}}^{t} \mathfrak{F}_{\tau} \circ \vec{X}_{\tau} d \tau
$$

We will call the linear differential equation

$$
\frac{d}{d t} \mathbb{G}_{t}=-\vec{X}_{t} \circ \mathfrak{G}_{t}
$$

the adjoint of (1.13); the integral equation

$$
\mathfrak{S}_{t}=\mathfrak{G}_{0}-\int_{t_{0}}^{t} \vec{X}_{\tau} \circ \mathfrak{G}_{\tau} d \tau
$$

corresponds to it. We will call (1.13), (1.15), (1.16), and (1.17) linear operator equations.

We will call an absolutely continuous family $\mathfrak{l}_{t}$ invertible if for all $t$ the operator $\mathfrak{l}_{t} \in \mathcal{L}(\Phi)$ has an inverse $\mathfrak{I}_{t}^{-1} \in \mathcal{L}(\Phi)$ and $\mathfrak{l}_{t}^{-1}$ is also absolutely continuous. In this case we will call $\mathfrak{I}_{t}$ and $\mathfrak{I}_{t}^{-1}$ mutual inverses. Every flow $\hat{P}_{t}$ is invertible, since it is possible to prove by the implicit function theorem that $P_{t}^{-1}$ depends absolutely continuously on $t$.

Proposition 1.1. 1) If the operator $\mathfrak{F}_{0}$ in (1.15) is a left inverse of $\mathfrak{S}_{0}$ in $(1.17)$, then every solution $\mathfrak{F}_{t}$ of $(1.15)$ is a left inverse of every solution $\mathscr{S}_{t}$ of $(1.17)$, i.e. $\mathfrak{F}_{t} \circ \mathscr{S}_{t}=\mathrm{Id}$ for all $t \in J_{t_{0}}$.

2) If $\mathfrak{F}_{0}$ is a left inverse of $\mathbb{S}_{0}$ and either one of the equations (1.15) or (1.17) has an invertible solution, then each equation has a unique solution, and the solutions are mutually inverse flows. Consequently if a solution of one of the equations is a flow, then the inverse flow is a solution of the other, and the solutions are unique.

Proof. Part 1) can be verified by direct differentiation:

$$
\frac{d}{d t}\left(\mathfrak{F}_{t} \circ \mathfrak{G}_{t}\right)=\left(\frac{d}{d t} \mathfrak{F}_{t}\right) \circ \mathfrak{G}_{t}+\mathfrak{F}_{t} \circ\left(\frac{d}{d t} \mathfrak{G}_{t}\right)=\mathfrak{F}_{t} \circ \vec{X}_{t} \circ \mathfrak{G}_{t}-\mathfrak{F}_{t} \circ \vec{X}_{t} \circ \mathfrak{G}_{t}=0
$$

consequently by virtue of the absolute continuity of $\mathfrak{F}_{t} \circ \mathbb{S}_{t}$ we have that $\mathfrak{F}_{t} \circ \mathbb{S}_{t}=$ $\mathfrak{F}_{0} \circ \mathfrak{S}_{0}=$ Id for all $t \in \mathbf{R}$. 
For the proof of part 2), we note first of all that a similar check shows that if $\mathfrak{l}_{t}$, $t \in J_{t_{0}}$, is an invertible solution of (1.15) or (1.17), then $\mathfrak{I}_{t}^{-1}$ is a solution of (1.17) or (1.15), respectively, and the equations have unique solutions.

Finally, from the invertibility and uniqueness it follows that $\mathfrak{F}_{t}$ and $\mathbb{F}_{t}$ are flows. In fact, the family of operators $\mathfrak{l}_{t}$ defined by

$$
\mathfrak{l}_{t} \varphi=\varphi \circ \mathfrak{G}_{t} E \quad \forall \varphi \in \Phi
$$

satisfy (1.16):

$$
\begin{gathered}
\left(\frac{d}{d t} \mathfrak{l}_{t}\right) \varphi=\frac{d}{d t}\left(\varphi \circ \mathfrak{S}_{t} E\right)=\left((\operatorname{grad} \varphi) \circ\left(\mathfrak{S}_{t} E\right) \cdot\left(-\vec{X}_{t} \circ \mathfrak{G}_{t} E\right)\right. \\
=-\vec{X}_{t}\left(\varphi \circ \mathfrak{G}_{t} E\right)=-\vec{X}_{t} \circ \mathfrak{l}_{t} \varphi ;
\end{gathered}
$$

therefore $\mathfrak{l}_{t}=\mathscr{S}_{t}$ and consequently $\mathscr{S}_{t}$ and $\mathfrak{F}_{t}$ are flows, since $\mathscr{S}_{t} \varphi=\varphi \circ \mathscr{S}_{t} E \forall \varphi \in \Phi$, and $\mathbb{S}_{t}^{-1}=\mathscr{F}_{t}$.

If the flow $\hat{P}_{t}$ satisfies the operator equation (1.13), then the corresponding flow $P_{t}$ in $\mathbf{R}^{n}$ is determined by the ordinary differential equation

$$
\frac{d x}{d t}=X_{t}(x)
$$

since

$$
\frac{d P_{t}}{d t}=\frac{d}{d t} \hat{P}_{t} E=\hat{P}_{t} \circ \vec{X}_{t} E=\hat{P}_{t} X_{t}=X_{t} \circ P_{t}
$$

If $\mathscr{S}_{t}$ is an arbitrary solution of (1.16) with initial condition $\mathscr{S}_{t_{0}}=\mathrm{Id}$, then for all $\varphi$ in $\Phi$ the function $(t, x) \mapsto \mathscr{G}_{t} \varphi(x)=\omega_{t}(x)$ satisfies the first-order linear homogeneous partial differential equation

$$
\frac{\partial \omega_{t}}{\partial t}+\sum_{\alpha=1}^{n} \frac{\partial \omega_{t}}{\partial x^{\alpha}} X^{\alpha}=\frac{\partial \omega_{t}}{\partial t}+\vec{X}_{t} \omega_{t}=0, \quad \omega_{t_{0}}(x)=\varphi(x) .
$$

Conversely, every absolutely continuous family of functions $\omega_{t}$ which satisfies this equation is representable in the form

$$
\omega_{t}=\hat{P}_{t}^{-1} \omega_{t_{0}}
$$

where $\hat{P}_{t}$ is a flow satisfying (1.13) and the initial condition $\hat{P}_{t_{0}}=\mathrm{Id}$, since

$$
\frac{d}{d t}\left(\hat{P}_{t} \varphi_{t}\right)=\left(\frac{d}{d t} \hat{P}_{t}\right) \varphi_{t}+\hat{P}_{t} \frac{d}{d t} \varphi_{t}=\hat{P}_{t} \circ \vec{X}_{t} \varphi_{t}-\hat{P}_{t} \circ \vec{X}_{t} \varphi_{t}=0 .
$$

In the next section we show that if the field $\vec{X}_{t}$ in (1.15) and (1.17) is locally integrable, then the equations have invertible solutions which, consequently, are unique, mutually inverse flows; we also give representations of the solutions in the form of "chronological exponentials." We note here only that every flow $\hat{P}_{t}$ can be represented in the form of a solution to both (1.13) and its adjoint (1.16), since

$$
\frac{d}{d t} \hat{P}_{t}=\hat{P}_{t} \circ\left(\hat{P}_{t}^{-1} \circ \frac{d}{d t} \hat{P}_{t}\right)=-\left(\left(-\frac{d}{d t} \hat{P}_{t}\right) \circ \hat{P}_{t}^{-1}\right) \circ \hat{P}_{t}
$$

and it can be verified by direct calculation that, for any fixed $t$, the quantities

$$
\hat{P}_{t}^{-1} \circ \frac{d}{d t} \hat{P}_{t}, \quad\left(\frac{d}{d t} \hat{P}_{t}\right) \circ \hat{P}_{t}^{-1}
$$

belong to $\operatorname{Der}(\Phi)$. 
Proposition 1.2. Assume that the family of diffeomorphisms $\left(\hat{P}_{t_{0}, t}\right)$ is defined for all $t_{0}$ and $t$ such that $\left|t-t_{0}\right|<\rho_{t_{0}}$ (where $\rho_{t_{0}}$ is an arbitrary function of $t_{0}$ ), that it depends absolutely continuously on $t_{0}$ and $t$, and that it satisfies the differential equation

$$
\frac{d}{d t} \hat{P}_{t_{0}, t}=\hat{P}_{t_{0}, t} \circ \vec{X}_{t}
$$

where $\vec{X}_{t}$ is an arbitrary (locally integrable) field, and the initial condition

$$
\hat{P}_{t, t}=\mathrm{Id} \quad \forall t \in \mathbf{R} \text {. }
$$

Then it is possible to define $\hat{P}_{t_{0}, t}$ as a solution of the given equation for arbitrary values of $t_{0}$ and $t$ in $\mathbf{R}$.

Proof. Suppose that it is possible to fix a point $t_{1}$ between $t_{0}$ and $t, t_{0}<t_{1}<t$, satisfying $\left|t_{1}-t_{0}\right|<\rho_{\tau_{0}}$ and $\left|t-t_{1}\right|<\rho_{t_{1}}$. The composition $\hat{Q}_{t_{0, t}}=\hat{P}_{t_{0, t_{1}}} \circ \hat{P}_{t_{1}, t}$ satisfies

$$
\frac{d}{d t} \hat{Q}_{t_{0}, t}=\hat{Q}_{t_{0}, t} \circ \vec{X}_{t}
$$

and the initial condition $\hat{Q}_{t_{0}, t_{1}}=\hat{P}_{t_{0}, t_{1}}$ and therefore by Proposition 1.1 does not depend on the choice of $t_{1}$. Similarly, the composition $\hat{P}_{t_{0}, t_{1}} \circ \hat{P}_{t_{1}, t_{2}} \circ \cdots \circ \hat{P}_{t_{m}, t}$ does not depend on the choice of $t_{1}, \ldots, t_{m}$. Consequently if, for arbitrary given $t_{0}$ and $t$ in $R$, we choose $t_{1}, \ldots, t_{m}$ so that $\left|t_{1}-t_{0}\right|<\rho_{t_{0}},\left|t_{2}-t_{1}\right|<\rho_{t_{1}}, \ldots,\left|t-t_{m}\right|<\rho_{t_{m}}$, and if we put $\hat{Q}_{t_{0, t}}=$ $\hat{P}_{t_{0}, t_{1}} \circ \hat{P}_{t_{1}, t_{2}} \circ \cdots \circ \hat{P}_{t_{m}, t}$, we get a well-defined family of diffeomorphisms $\hat{Q}_{t_{0}, t}, t_{0}$, $t \in \mathbf{R}$, which depends absolutely continuously on $t_{0}$ and $t$, which satisfies the given differential equation and the initial condition $\hat{Q}_{t, t}=$ Id for all $t$ in $\mathbf{R}$, and which coincides with $\hat{P}_{t_{0}, t}$ for $\left|t-t_{0}\right|<\rho_{t_{0}}$.

In what follows we will represent flows in the form of families of diffeomorphisms $\hat{P}_{t_{0}, t}$ which depend absolutely continuously on the parameters $t_{0}$ and $t$, and which reduce to the identity mapping for $t_{0}=t, \hat{P}_{t, t}=$ Id. Similarly, we will solve the equations

$$
\begin{gathered}
\widetilde{F}_{t_{0}, t}=\mathrm{Id}+\int_{t_{0}}^{t} \mathfrak{F}_{t_{0}, \tau} \circ \vec{X}_{\tau} d \tau, \\
\mathfrak{S}_{t_{0}, t}=\mathrm{Id}-\int_{t_{0}}^{t} \vec{X}_{\tau} \circ \mathfrak{S}_{t_{0}, \tau} d \tau=\mathrm{Id}+\int_{t_{0}}^{t} \vec{Y}_{\tau} \circ \mathfrak{S}_{t_{0}, \tau} d \tau .
\end{gathered}
$$

In the notation we have adopted,

$$
\hat{P}_{t_{0}, t_{1}} \circ \hat{P}_{t_{1}, t_{2}} \circ \ldots \circ \hat{P}_{t_{m}, t}=\hat{P}_{t_{0}, t} .
$$

5. The algebra of formal chronological series. Typical examples of chronological series are so-called Volterra series, which arise if we solve (1.18) and (1.19) formally by means of successive substitutions. As "formal solutions" of (1.18) and (1.19) we get the formal series

$$
\begin{gathered}
\mathfrak{F}_{t_{0}, t}=\mathrm{Id}+\int_{t_{0}}^{t} d \tau_{1} \vec{X}_{\tau_{1}}+\int_{t_{0}}^{t} d \tau_{1} \int_{t_{0}}^{\tau_{1}} d \tau_{2} \vec{X}_{\tau_{2}} \circ \vec{X}_{\tau_{1}}+\ldots \\
=\mathrm{Id}+\sum_{m=1}^{\infty} \int_{t_{0}}^{t} d \tau_{1} \int_{t_{0}}^{\tau_{1}} d \tau_{2} \ldots \int_{t_{0}}^{\tau_{m-1}} d \tau_{m} \vec{X}_{\tau_{m}} \circ \ldots \circ \vec{X}_{\tau_{1}}, \\
\mathcal{G S}_{t_{0}, t}=\mathrm{Id}+\sum_{m=1}^{\infty} \int_{t_{0}}^{t} d \tau_{1} \int_{t_{0}}^{\tau_{1}} d \tau_{2} \ldots \int_{t_{0}}^{\tau_{m-1}} d \tau_{m} \vec{Y}_{\tau_{1}} \circ \ldots \circ \vec{Y}_{\tau_{m}} .
\end{gathered}
$$


The general term of the first series is an iterated integral in which the variables of integration in the expression $\vec{X}_{\tau_{m}} \circ \cdots \circ \vec{X}_{\tau_{1}}$ increase from left to right, $t_{0}<\tau_{m}<\tau_{m-1}$ $<\cdots<\tau_{1}<t$. We call this series a right formal Volterra chronological series and denote it by

$$
\vec{\vartheta}_{t_{0}, t}\left(\vec{X}_{\tau}\right)=\operatorname{Id}+\sum_{m=1}^{\infty} \int_{t_{0}}^{t} d \tau_{1} \int_{t_{0}}^{\tau_{1}} d \tau_{2} \ldots \int_{t_{0}}^{\tau_{m-1}} d \tau_{m} \vec{X}_{\tau_{m}} \circ \ldots \circ \vec{X}_{\tau_{1}},
$$

where the arrow over the $\mathcal{V}$ indicates the direction of increase of the variables in the expression $\vec{X}_{\tau_{m}} \circ \ldots \circ \vec{X}_{\tau_{1}}$. Similarly, we call

$$
\overleftarrow{\mathfrak{V}}_{t_{0}, t}\left(\vec{Y}_{\tau}\right)=\mathrm{Id}+\sum_{m=1}^{\infty} \int_{t_{0}}^{t} d \tau_{1} \int_{t_{0}}^{\tau_{1}} d \tau_{2} \ldots \int_{i_{0}}^{\tau_{m-1}} d \tau_{m} \vec{Y}_{\tau_{1}} \circ \ldots \circ \vec{Y}_{\tau_{m}}
$$

a left formal Volterra chronological series.

We now construct the ring of general formal chronological series.

In an $m$-dimensional space with points $\tau^{(m)}=\left(\tau_{1}, \ldots, \tau_{m}\right)$ we denote the simplex

$$
\left\{\tau^{(m)}=\left(\tau_{1}, \ldots, \tau_{m}\right) \mid t_{0} \leqslant \tau_{m} \leqslant \ldots \leqslant \tau_{1} \leqslant t\right\}
$$

by $\Delta_{t_{0}, t}\left(\tau^{(m)}\right)=\Delta\left(\tau^{(m)}\right)=\Delta\left(\tau_{1}, \ldots, \tau_{m}\right)$. If $\pi$ is an arbitrary permutation of $1, \ldots, m$, then we will denote the simplex

$$
\Delta\left(\pi \tau^{(m)}\right)=\Delta\left(\tau_{\pi(1)}, \ldots, \tau_{\pi(m)}\right)
$$

by

$$
\left\{\left(\tau_{1}, \ldots, \tau_{m}\right) \mid t_{0} \leqslant \tau_{\pi(m)} \leqslant \ldots \leqslant \tau_{\pi(1)} \leqslant t\right\} .
$$

The collection of all simplexes $\Delta\left(\pi \tau^{(m)}\right)$ corresponding to all possible permutations $\pi$ gives the triangulation of a cube

$$
B_{t_{0}, t}^{(m)}=\left\{\left(\tau_{1}, \ldots, \tau_{m}\right) \mid t_{0} \leqslant \tau_{1} \leqslant t, \ldots, t_{0} \leqslant \tau_{m} \leqslant t\right\},
$$

as is easy to see. Let $S(j, k), j+k=m$, be the set of all permutations of $1, \ldots, m$ which preserve the order of the first $j$ numbers and, separately, of the last $k$ numbers; $S(j, k)$ obviously consists of $m ! / j ! k$ ! elements. It can be verified directly that

$$
\Delta\left(\tau^{(j)}\right) \times \Delta\left(\tau^{(k)}\right)=\bigcup_{\pi \in S(j, k)} \Delta\left(\pi^{-1} \tau^{(m)}\right) .
$$

Let $\mathfrak{L}\left(\tau^{(m)}\right)=\mathfrak{L}\left(\tau_{1}, \ldots, \tau_{m}\right)$ be an integrable function on the cube $B_{t_{0}, t}^{m}$ with values in $\mathcal{L}(\Phi)$. Here, as in subsection 3, we understand integrability in the "weak" sense. The obvious equality between the iterated integral and the $m$-fold integral over the simplex $\Delta\left(\tau^{(m)}\right)$ holds:

$$
\int_{0_{0}}^{t} d \tau_{1} \int_{t_{0}}^{\tau_{1}} d \tau_{2} \ldots \int_{i_{0}}^{\tau_{m-1}} d \tau_{m} \mathfrak{Q}\left(\tau^{(m)}\right)=\int_{\Delta\left(\tau^{(m)}\right)} \mathfrak{L}\left(\tau^{(m)}\right) d \tau^{(m)}
$$

and a change of variables gives

$$
\int_{\Delta\left(\pi^{-1} \tau^{(m)}\right)} \mathfrak{L}\left(\tau^{(m)}\right) d \tau^{(m)}=\int_{\Delta\left(\tau^{(m)}\right)} \mathfrak{L}\left(\pi \tau^{(m)}\right) d \tau^{(m)}=\int_{\Delta\left(\tau^{(m)}\right)} \mathfrak{L}\left(\tau_{\pi(1)}, \ldots, \tau_{\pi(m)}\right) d \tau^{(m)}
$$


If $\mathfrak{F}\left(\tau^{(j)}\right)$ and $\mathscr{S}\left(\tau^{(k)}\right)$ are two integrable functions, then by (1.23) and (1.24) we can write

$$
\begin{aligned}
\int_{\Delta\left(\tau^{(j)}\right)} \mathfrak{F}\left(\tau^{(j)}\right) d \tau^{(j)} \circ \int_{\Delta\left(\tau^{(k)}\right)} \mathfrak{S}\left(\tau^{(k)}\right) d \tau^{(k)}=\int_{\Delta\left(\tau^{(j)}\right) \times \Delta\left(\tau^{(k)}\right)} & F\left(\tau^{(j)}\right) \circ \mathscr{S}\left(\tau^{(k)}\right) d \tau^{(j)} \otimes d \tau^{(k)} \\
& =\sum_{\pi \in S(j, k)} \int_{\Delta\left(\pi^{-1} \tau^{(m)}\right)} \mathfrak{F}\left(\tau_{1}, \ldots, \tau_{j}\right) \circ \mathbb{S}\left(\tau_{j \neq \mathbf{1}}, \ldots, \tau_{m}\right) d \tau^{(m)} \\
& =\sum_{\pi \in S(j, k)} \int_{\Delta\left(\tau^{(m)}\right)} \mathfrak{F}\left(\tau_{\pi(1)}, \ldots, \tau_{\pi(j)}\right) \circ \mathscr{H}\left(\tau_{\pi(j+1)}, \ldots, \tau_{\pi(m)}\right) d \tau^{(m)},
\end{aligned}
$$

and taking (1.24) into account, we get

$$
\begin{gathered}
\int_{i_{0}}^{t} d \tau_{1} \int_{i_{0}}^{\tau_{1}} d \tau_{2} \ldots \int_{i_{0}}^{\tau_{j-1}} d \tau_{j} \mathfrak{F}\left(\tau_{1}, \ldots, \tau_{j}\right) \circ \int_{t_{0}}^{t} d \tau_{1} \int_{i_{0}}^{\tau_{1}} d \tau_{2} \ldots \int_{t_{0}}^{\tau_{k-1}} d \tau_{k} \mathbb{S}\left(\tau_{1}, \ldots, \tau_{k}\right) \\
=\sum_{\pi \in S(j, k)} \int_{t_{0}}^{t} d \tau_{1} \int_{t_{0}}^{\tau_{1}} d \tau_{2} \ldots \int_{t_{0}}^{\tau_{m-1}} d \tau_{m} \mathfrak{F}\left(\tau_{\pi(1)}, \ldots, \tau_{\pi(j)}\right) \circ \mathbb{S}\left(\tau_{\pi(j+1)}, \ldots, \tau_{\pi(m))} .\right.
\end{gathered}
$$

A formal series of the form

$$
\mathfrak{S}_{t}\left(\vec{X}_{\tau}\right)=\mathfrak{P}_{0}\left(\vec{X}_{t}\right)+\sum_{m=1}^{\infty} \int_{i_{0}}^{t} d \tau_{1} \int_{t_{0}}^{\tau_{1}} d \tau_{2} \int_{i_{0}}^{\tau_{2}} d \tau_{3} \ldots \int_{i_{0}}^{\tau_{m-1}} d \tau_{m} \mathfrak{P}_{m}\left(\vec{X}_{t}, \vec{X}_{\tau_{1}}, \ldots, \vec{X}_{\tau_{m}}\right)
$$

where $\mathfrak{P}_{m}\left(\zeta_{0}, \ldots, \zeta_{m}\right)$ is a polynomial in the "noncommutative variables" $\zeta_{0}, \ldots, \zeta_{m}$, $m=1,2, \ldots$, and $X_{t}, t \in \mathbf{R}$, is a vector field, is called a formal chronological series.

It is easy to see that the natural definitions of the operations of addition, multiplication by a real number, and integration of formal chronological series from $t_{0}$ to $t$ again give chronological series. The "Cauchy product" of chronological series,

$$
\begin{gathered}
\mathfrak{S}_{t}^{\prime}\left(\vec{X}_{\tau}\right) \circ \mathfrak{S}_{t}^{\prime \prime}\left(\vec{X}_{\tau}\right)=\mathfrak{P}_{0}^{\prime}\left(\vec{X}_{t}\right) \circ \mathfrak{P}_{0}^{\prime \prime}\left(\vec{X}_{t}\right) \\
+\sum_{m=1}^{\infty} \sum_{\alpha+\beta=m} \int_{t_{0}}^{t} d \tau_{1} \int_{i_{0}}^{\tau_{1}} d \tau_{2} \ldots \int_{i_{0}}^{\tau_{\alpha-1}} d \tau_{\alpha} \mathfrak{P}_{\alpha}^{\prime}\left(\vec{X}_{t}, \vec{X}_{\tau_{1}}, \ldots, \vec{X}_{\tau_{\alpha}}\right) \\
\circ \int_{i_{0}}^{t} d \tau_{1} \int_{t_{0}}^{\tau_{1}} d \tau_{2} \ldots \int_{i_{0}}^{\tau_{\beta}-1} d \tau_{\beta} \mathfrak{P}_{\beta}^{\prime \prime}\left(\vec{X}_{t}, \vec{X}_{\tau_{1}}, \ldots, \vec{X}_{\tau_{\beta}}\right),
\end{gathered}
$$

reduces by (1.26) to the chronological series (1.27), where

$$
=\sum_{\alpha+\beta=m} \sum_{\pi \in S(j, k)} \mathfrak{P}_{\alpha}^{\prime}\left(\zeta_{0}, \zeta_{\pi(1)}, \ldots, \zeta_{\pi(j)}\right) \circ \mathfrak{P}_{\beta}^{\prime \prime}\left(\zeta_{0}, \zeta_{\pi(j+1)}, \ldots, \zeta_{\pi(m)}\right) .
$$

Thus the set of all formal chronological series becomes a real associative algebra with the multiplication given by (1.28).

The right and left Volterra series (1.21) and (1.22) are special cases of formal chronological series. From the obvious identities 


$$
\begin{gathered}
\int_{t_{0}}^{t} d \tau_{1} \int_{t_{0}}^{\tau_{1}} d \tau_{2} \ldots \int_{t_{0}}^{\tau_{m-1}} d \tau_{m} \vec{X}_{\tau_{m}} \circ \ldots \circ \vec{X}_{\tau_{1}}=\int_{\Delta_{t_{0}, t}\left(\tau^{(m)}\right)} \vec{X}_{\tau_{m}} \circ \ldots \circ \vec{X}_{\tau_{1}} d \tau^{(m)}, t_{0} \leqslant t, \\
\int_{t_{0}}^{t} d \tau_{1} \int_{t_{0}}^{\tau_{1}} d \tau_{2} \ldots \int_{t_{0}}^{\tau_{m-1}} d \tau_{m} \vec{X}_{\tau_{m}} \circ \cdots \circ \vec{X}_{\tau_{1}}=\int_{\Delta_{t, t_{0}}\left(\tau^{(m)}\right)}(-1)^{m} \vec{X}_{\tau_{1}} \circ \ldots \circ \vec{X}_{\tau_{m}} d \tau^{(m)}, \\
t \leqslant t_{0},
\end{gathered}
$$

and the analogous identities

$$
\begin{aligned}
\int_{t_{0}}^{t} d \tau_{1} \int_{t_{0}}^{\tau_{1}} d \tau_{2} \ldots & \int_{t_{0}}^{\tau_{m \rightarrow 1}} d \tau_{m} \vec{X}_{\tau_{1}} \circ \ldots \circ \vec{X}_{\tau_{m}}=\int_{\Delta_{t_{0}, t}\left(\tau^{(m)}\right)} \vec{X}_{\tau_{1}} \circ \ldots \circ \vec{X}_{\tau_{m}} d \tau^{(m)}, \quad t_{0} \leqslant t, \\
= & \int_{t_{0}}^{t} d \tau_{1} \int_{t_{t, t_{0}}\left(\tau^{(m)}\right)}^{\tau_{1}} d \tau_{2} \ldots \int_{t_{0}}^{\tau_{m-1}} d \tau_{m} \vec{X}_{\tau_{1}} \circ \ldots \circ \vec{X}_{\tau_{m}}(-1)^{m} \vec{X}_{\tau_{m}} \circ \cdots \circ \vec{X}_{\tau_{1}} d \tau^{(m)}, \quad t \leqslant t_{0},
\end{aligned}
$$

we get the important relation

$$
\overrightarrow{\mathscr{V}}_{t_{0}, t}\left(\vec{X}_{\tau}\right)=\overleftarrow{\mathscr{V}}_{t, t_{0}}\left(-\vec{X}_{\tau}\right)
$$

i.e., every right Volterra series in the field $\vec{X}_{t}$ is at the same time a left Volterra series in $-\vec{X}_{t}$.

In conclusion, we note that if the fields $\vec{X}_{t^{\prime}}$ and $\vec{X}_{t^{\prime \prime}}$ commute for arbitrary $t^{\prime}$ and $t^{\prime \prime}$, and if we put $t_{0}<t$ for definiteness, we get that

$$
\begin{gathered}
\int_{t_{0}}^{t} d \tau_{1} \int_{t_{0}}^{\tau_{1}} d \tau_{2} \cdots \int_{t_{0}}^{\tau_{m-1}} d \tau_{m} \vec{X}_{\tau_{m}} \circ \cdots \circ \vec{X}_{\tau_{1}}=\int_{\Delta\left(\tau^{(m)}\right)} \vec{X}_{\tau_{m}} \circ \cdots \circ \vec{X}_{\tau_{1}} d \tau^{(m)} \\
=\frac{1}{m !} \sum_{\pi} \int_{\Delta\left(\pi \tau^{(m))}\right.} \vec{X}_{\tau_{1}} \circ \cdots \circ \vec{X}_{\tau_{m}} d \tau^{(m)} \\
=\frac{1}{m !} \int_{B_{t_{0}, t}^{(m)}} \vec{X}_{\tau_{1}} \circ \cdots \circ \vec{X}_{\tau_{m}} d \tau^{(m)}=\frac{1}{m !}\left(\int_{t_{0}}^{t} \vec{X}_{\tau} d \tau\right)^{m} .
\end{gathered}
$$

Consequently in the "commutative" case considered here, left and right Volterra series coincide and are representable as formal power series:

$$
\vec{\vartheta}_{t_{0}, t}\left(\vec{X}_{\tau}\right)=\overleftarrow{\mho}_{t_{0}, t}\left(\vec{X}_{\tau}\right)=\sum_{m=0}^{\infty} \frac{1}{m !}\left(\int_{t_{0}}^{t} \vec{X}_{\tau} d \tau\right)^{m}
$$

\section{§2. Summation of Volterra series and exponential} representation of flows

In this section we prove that the operator equations (1.18) and (1.19) have unique solutions which are flows. We construct flows with the help of a device we call summation of Volterra series. 
1. The case of an analytic field. We denote by $V_{\sigma}$ a complex $\sigma$-neighborhood of the real space $\mathbf{R} \subset \mathbf{C}^{n}$ :

$$
V_{\sigma}=\left\{z=\left(\begin{array}{c}
z_{1} \\
\vdots \\
z^{n}
\end{array}\right) \in \mathrm{C}^{n}|| \operatorname{Im} z \mid \leqslant \sigma, \sigma>0\right\}, \quad|\operatorname{Im} z|=\max _{\alpha}\left|\operatorname{Im} z^{\alpha}\right|,
$$

and by $\Omega_{\sigma}$ the set of all real analytic functions $\omega$ on $\mathbf{R}^{n}$ whose domains can be extended to $V_{\sigma}$ in such a way that the extended functions are (complex) analytic and bounded on $V_{\sigma}$. We will again denote the extension to a complex neighborhood by the same symbol, $\omega$, although (if we were to be completely precise) we should use some new symbol such as $\tilde{\omega}$, for example; this applies also to the partial derivatives $\partial_{\alpha} \omega$ and their complex extensions $\widetilde{\partial_{\alpha} \omega}=\partial \tilde{\omega} / \partial z^{\alpha}$.

We make $\Omega_{\sigma}$ into a Banach space by defining a norm

$$
\|\omega\|_{\sigma}^{c^{n}}=\sup _{z \in V_{\sigma}}|\omega(z)|, \quad \omega \in \Omega_{\sigma},
$$

in it. We denote the Cartesian product of $n$ copies of $\Omega_{\sigma}$ by $\Omega_{\sigma}^{n}$.

We will call the field $\vec{X}_{t}, t \in \mathbf{R}$, bounded analytic if for some $\sigma>0$ we have $X_{t} \in \Omega_{\sigma}^{n}$ for all $t \in \mathbf{R}$; we put

$$
\left\|X_{t}\right\|_{\sigma}^{c^{n}}=\max _{\alpha}\left\|X_{t}^{\alpha}\right\|_{\sigma}^{\mathbf{c}^{n}}
$$

We define a bounded analytic mapping analogously, and in particular a bounded analytic diffeomorphism $P: \mathbf{R}^{n} \rightarrow \mathbf{R}^{n}$ which corresponds to the operator $\hat{P} \in \mathcal{E}(\Phi)$ with the same name.

Proposition 2.1. Suppose that $\vec{X}_{t}, t \in \mathbf{R}$, is a bounded, analytic, uniformly integrable field, and that $X_{t} \in \Omega_{\sigma}^{n}$ for all $t \in \mathbf{R}$. Then for all $\sigma^{\prime}<\sigma$ there is a number $\rho>0$ such that for all $\omega \in \Omega_{0}$ the series

$$
\overrightarrow{\mathcal{V}}_{t_{\mathrm{e}}, t}\left(\vec{X}_{\tau}\right) \omega(z)=\omega(z)+\sum_{m=1}^{\infty} \int_{t_{0}}^{t} d \tau_{1} \int_{t_{0}}^{\tau_{1}} d \tau_{2} \ldots \int_{t_{0}}^{\tau_{m}-1} d \tau_{m} \vec{X}_{\tau_{m}} \circ \ldots \circ \vec{X}_{\tau_{2}} \omega(z)
$$

converges absolutely and uniformly with respect to $t_{0}$, $t$, and $z$ for $\left|t-t_{0}\right| \leqslant \rho, z \in V_{\sigma^{\prime}}$, and

$$
\overrightarrow{\mathfrak{V}}_{t_{0}, t}\left(\vec{X}_{\tau}\right)\left(\omega_{1} \omega_{2}\right)=\overrightarrow{\widetilde{V}}_{t_{0}, t}\left(\vec{X}_{\tau}\right) \omega_{1} \overrightarrow{\mathcal{V}}_{t_{0}, t,}\left(\vec{X}_{\tau}\right) \omega_{2} \quad \forall \omega_{1}, \omega_{2} \in \Omega_{\sigma}
$$

Proof. Taking into account the analyticity of $X_{t}$ and making use of the Cauchy integral representation, we prove the estimate

$$
\begin{gathered}
\left\|\int_{t_{0}}^{t} d \tau_{1} \int_{t_{0}}^{\tau_{1}} d \tau_{2} \ldots \int_{t_{0}}^{\tau_{m-1}} d \tau_{m}^{-} \vec{X}_{\tau_{m}} \circ \ldots \circ \vec{X}_{\tau_{1}} \omega\right\|_{\sigma^{\prime}}^{\mathbf{C}^{n}} \\
\leqslant\left(\frac{2 n}{\sigma-\sigma^{\prime}}\right)^{m}\left|\int_{t_{0}}^{t}\left\|X_{\tau}\right\|_{\sigma}^{\mathbf{C}^{n}} d \tau\right|^{m}\|\omega\|_{\sigma}^{\mathbf{C}^{n}},
\end{gathered}
$$

from which the absolute and uniform convergence of (2.1) follows immediately.

The Cauchy formula

$$
X_{t}(z)=\frac{1}{(2 \pi i)^{n}} \int_{C_{1}} d w^{1} \ldots \int_{C_{n}} d w^{n} \frac{X_{t}(z)}{\left(w^{1}-z^{1}\right) \ldots\left(w^{n}-z^{n}\right)}
$$


where $C_{\alpha}$ is a circle with center at $z^{\alpha}$ and with a sufficiently small radius, allows us to represent $\vec{X}_{\tau_{\alpha}}$ in the form

$$
\vec{X}_{\tau_{\alpha}}=\sum_{k_{\alpha}=1}^{n} \frac{1}{(2 \pi i)^{n}} \int_{C_{1}} d w_{\alpha}^{1} \cdots \int_{c_{n}} d w_{\alpha}^{n} \frac{X_{\tau_{\alpha}}^{k}\left(w_{\alpha}^{\prime}\right)}{\Pi_{w_{\alpha}}} \partial_{k_{\alpha}} .
$$

Here $\Pi_{w_{\alpha}}$ is a function of $z$,

$$
\Pi_{w_{\alpha}}(z)=\frac{1}{\left(w_{\alpha}^{1}-z^{1}\right) \ldots\left(w_{\alpha}^{n}-z^{n}\right)},
$$

where the complex vector $w_{\alpha}$ with coordinates $w_{\alpha}^{1}, \ldots, w_{\alpha}^{n}$ plays the role of a parameter. We have

$$
\begin{gathered}
\vec{X}_{\tau_{m}} \circ \ldots \circ \vec{X}_{\tau_{1}} \omega=\sum_{k_{1}, \ldots, k_{m}=1}^{n} X_{\tau_{m}}^{k_{m}} \partial_{k_{m}} \circ \ldots \circ X_{\tau_{1}}^{k_{1}} \partial_{k_{1}} \omega \\
=\frac{1}{(2 \pi i)^{n(1+m)}} \sum_{k_{1}, \ldots, k_{m}=1}^{n} \int_{C_{1}} d w_{m}^{1} \ldots \int_{C_{n}} d w_{m}^{n} \ldots \int_{C_{1}} d w_{1}^{1} \ldots \int_{C_{m}} d w_{1}^{n} \int_{C_{1}} d w^{1} \\
\ldots \int_{C_{n}} d w^{n} X_{\tau_{m}}^{k_{m}}\left(w_{m}\right) \ldots X_{\tau_{1}}^{k_{1}}\left(w_{1}\right) \omega(w) \chi_{w_{m}, \ldots, w_{1}, w}^{k_{m}, \ldots, k_{1}},
\end{gathered}
$$

where

$$
\chi_{w^{\prime} m, \ldots, w_{1}, w}^{k_{m}, \ldots, k_{1}}=\frac{1}{\Pi_{w_{m}}} \partial_{k_{m}} \frac{1}{\Pi_{w_{m-1}}} \partial_{k_{m-1}} \ldots \frac{1}{\Pi_{w_{1}}} \partial_{k_{1}} \frac{1}{\Pi_{w^{\prime}}} .
$$

If $\left|w_{\alpha}-z\right| \geqslant \delta, \alpha=1, \ldots, m$, and $|w-z| \geqslant \delta$, then an obvious induction on $m$ gives that

$$
\left|\chi_{w_{m}, \ldots, w_{1}, w}^{k_{m}, \ldots, k_{1}}(z)\right| \leqslant(2 m-1) ! ! \frac{1}{\delta^{n} \delta^{(1+n) m}}<\frac{2^{m} m !}{\delta^{n} \delta^{(1+n) m}}
$$

therefore, assuming that $C_{\alpha}$ in (2.4) is a circle of radius $\sigma-\sigma^{\prime}$ with center at $z^{\alpha}$, we get, for all $z \in V_{\sigma^{\prime}}$,

$$
\begin{gathered}
\left|\vec{X}_{\tau_{m}} \circ \ldots \circ \vec{X}_{\tau_{1}} \omega(z)\right| \\
\leqslant \frac{n^{m}}{(2 \pi)^{n(1+m)}}\left\|X_{\tau_{m}}\right\|_{\sigma}^{C^{n}} \ldots\left\|X_{\tau_{1}}\right\|_{\sigma}^{C^{n}}\|\omega\|_{\sigma}^{C^{n}} \frac{2^{m} m !\left(2 \pi\left(\sigma-\sigma^{\prime}\right)\right)^{n(1+m)}}{\left(\sigma-\sigma^{\prime}\right)^{n}\left(\sigma-\sigma^{\prime}\right)^{(1+n) m}} \\
=m !\left(\frac{2 n}{\sigma-\sigma^{\prime}}\right)^{m}\left\|X_{\tau_{m}}\right\|_{\sigma}^{C^{n}} \ldots\left\|X_{\tau_{1}}\right\|_{\sigma}^{C^{n}}\|\omega\|_{\sigma}^{C^{n}} .
\end{gathered}
$$

Consequently

$$
\left\|\vec{X}_{\tau_{m}} \circ \ldots \circ \vec{X}_{\tau_{1}} \omega\right\|_{\sigma^{\prime}}^{\mathbf{C}^{n}} \leqslant m !\left(\frac{2 n}{\sigma-\sigma^{\prime}}\right)^{m}\left\|X_{\tau_{m}}\right\|_{\sigma}^{C^{n}} \ldots\left\|X_{\tau_{1}}\right\|_{\sigma}^{\mathbf{C}^{n}}\|\omega\|_{\sigma}^{\mathbf{C}^{n}}
$$

in particular,

$$
\begin{gathered}
\left\|\vec{X}_{\tau_{m}} \circ \ldots \circ \vec{X}_{\tau_{1}} E\right\|_{\sigma^{\prime}}^{\mathbf{C}^{n}}=\max _{\alpha}\left\|\vec{X}_{\tau_{m}} \circ \ldots \circ \vec{X}_{\tau_{2}} X_{\tau_{1}}^{\alpha}\right\|_{\sigma^{\prime}}^{\mathbf{C}^{n}} \\
\leqslant(m-1) !\left(\frac{2 n}{\sigma-\sigma^{\prime}}\right)^{m b-1}\left\|X_{\tau_{m}}\right\| \sigma \sigma^{\mathbf{C}^{n}} \cdots\left\|X_{\tau_{1}}\right\|_{\sigma}^{\mathbf{C}^{n}} .
\end{gathered}
$$


Thus, using the identity

$$
\int_{t_{0}}^{t} d \tau_{1} \int_{t_{0}}^{\tau_{1}} d \tau_{2} \ldots \int_{t_{0}}^{\tau_{m-1}} d \tau_{m}\left\|X_{\tau_{m}}\right\|_{\sigma}^{\mathbf{C}^{n}} \ldots\left\|X_{\tau_{1}}\right\|_{\sigma}^{\mathbf{C}^{n}}=\frac{1}{m !}\left(\int_{t_{0}}^{t}\left\|X_{\tau}\right\| \|_{\sigma}^{\mathbf{C}^{n}} d \tau\right)^{m}
$$

and taking into account the uniform integrability of $\vec{X}_{t}$, we get (2.3).

For the proof of the multiplicative property (2.2) of Volterra series, we note that an obvious induction gives the following generalization of the Leibniz rule for arbitrary fields $\vec{Y}_{1}, \ldots, \vec{Y}_{m}$ :

$$
\vec{Y}_{m} \circ \cdots \circ \vec{Y}_{1}\left(\varphi_{1} \varphi_{2}\right)=\sum_{j+k=m} \sum_{\pi \in S(j, k)}\left(\vec{Y}_{\pi(m)} \circ \cdots \circ \vec{Y}_{\pi(j+1)} \varphi_{1}\right)\left(\vec{Y}_{\pi(j)} \circ \cdots \circ \vec{Y}_{\pi(1)} \varphi_{2}\right),
$$

where $S(j, k)$ is defined in $\S 1.5$. Hence we get from (1.26) the following identity for $m=1,2, \ldots$, from which $(2.2)$ follows immediately:

$$
\begin{gathered}
\int_{t_{0}}^{t} d \tau_{1} \int_{t_{0}}^{\tau_{1}} d \tau_{2} \ldots \int_{t_{0}}^{\tau_{m-1}} d \tau_{m} \vec{X}_{\tau_{m}} \circ \ldots \circ \vec{X}_{\tau_{1}}\left(\omega_{1} \omega_{2}\right) \\
=\sum_{i+k=m} \int_{t_{0}}^{t} d \tau_{1} \int_{t_{0}}^{\tau_{1}} d \tau_{2} \ldots \int_{t_{0}}^{\tau_{m-1}} d \tau_{m} \sum_{\pi \in S(j, k)}\left(\vec{X}_{\tau_{\pi(m)}} \circ \ldots \circ \vec{X}_{\tau_{\pi(j+1)}} \omega_{1}\right) \\
\times\left(\vec{X}_{\tau_{\pi(i)}} \circ \ldots \circ \vec{X}_{\tau_{\pi(1)}} \omega_{2}\right)=\sum_{j+k=m} \int_{t_{0}}^{t} d \tau_{1} \int_{\tau_{0}}^{\tau_{1}} d \tau_{2} \ldots \int_{\tau_{0}}^{\tau_{j-1}} d \tau_{j} \vec{X}_{\tau_{j}} \circ \ldots \circ \vec{X}_{\tau_{1}} \omega_{1} \\
\times \int_{t_{0}}^{t} d \tau_{1} \int_{t_{0}}^{\tau_{1}} d \tau_{2} \ldots \int_{t_{0}}^{\tau_{k-1}} d \tau_{k} \vec{X}_{\tau_{k}} \circ \ldots \circ \vec{X}_{\tau_{1} \omega_{2} .}
\end{gathered}
$$

The proposition we have just proved allows us to assert that if $\vec{X}_{t}$ is a bounded analytic and uniformly integrable field, then it is possible to find $\rho>0$ such that for $\left|t-t_{0}\right| \leqslant \rho$ the mapping

$$
\overrightarrow{\mathfrak{V}}_{t_{0}, t}\left(\vec{X}_{\mathfrak{\tau}}\right) E: \mathbf{R}^{n} \rightarrow \mathbf{R}^{n}
$$

is also bounded analytic, and the identity

$$
\overrightarrow{\mathcal{V}}_{t_{0}, t}\left(\vec{X}_{\tau}\right) \omega=\omega \circ \overrightarrow{\mathcal{V}}_{t_{0}, t}\left(\vec{X}_{\tau}\right) E \quad \forall \omega \in \Omega_{\sigma}
$$

and its corollary

$$
\overrightarrow{\mathcal{V}}_{t_{\mathrm{t}, t}}\left(\vec{X}_{\tau}\right) X_{t}=X_{t} \circ \overrightarrow{\mathscr{V}}_{t_{0}, t}\left(\vec{X}_{\tau}\right) E
$$

both hold. The analyticity is obvious, and the identities follow from the facts that $\omega$ can be expanded in a series in powers of the independent variables, the operator $\overrightarrow{\widetilde{V}}_{t_{0, t}}\left(\vec{X}_{\tau}\right)$ can be applied termwise, and the result for each term follows immediately from (2.2).

If we put $P_{t_{0}, t}=\overrightarrow{\widetilde{V}}_{t_{0}, t}\left(\vec{X}_{\tau}\right) E$, we can see from (2.5) and the identities

$$
\overrightarrow{\mathcal{V}}_{t_{0}, t}\left(\vec{X}_{\tau}\right) E=E+\int_{t_{0}}^{t} \overrightarrow{\mathcal{V}}_{t_{0}, \tau}\left(\vec{X}_{\theta}\right) \circ \vec{X}_{\tau} E d \tau=E+\int_{t_{0}}^{t} \overrightarrow{\mathcal{V}}_{t_{0}, \tau}\left(\vec{X}_{\theta}\right) X_{\tau} d \tau,
$$


that the family $\hat{P}_{t_{0}, t},\left|t-t_{0}\right|<\rho$, satisfies

$$
\hat{P}_{t_{0}, t}=\mathrm{Id}+\int_{t_{0}}^{t} \hat{P}_{t_{0}, \tau} \circ \vec{X}_{\tau} d \tau .
$$

Proposition 2.1 continues to hold if we replace the right Volterra series by a left series. In this case (2.6) must be replaced by

$$
\overleftarrow{\mho}_{t_{0}, t}\left(\vec{X}_{\tau}\right) E=E+\int_{t_{0}}^{t} \vec{X}_{\tau} \circ \overleftarrow{\mho}_{t_{0}, \tau}\left(\vec{X}_{\theta}\right) E d \tau
$$

or the equivalent relation

$$
\frac{d}{d t} \overleftarrow{\mho}_{t_{0}, t}\left(\vec{X}_{\tau}\right) E=\vec{X}_{t} \overleftarrow{\mho}_{t_{0}, t}\left(\vec{X}_{\tau}\right) E
$$

The family of operators $\hat{Q}_{t_{0} t},\left|t-t_{0}\right| \leqslant \rho$, which satisfies the integral equation

$$
\hat{Q}_{t_{0}, t}=\mathrm{Id}-\int_{t_{0}}^{t} \vec{X}_{\tau} \circ \hat{Q}_{t_{0}, \tau} d \tau
$$

corresponds to the family of mappings

$$
Q_{t_{0}, t}=\overleftarrow{\mho}_{t_{0}, t}\left(-\vec{X}_{\tau}\right) E: \mathbf{R}^{n} \rightarrow \mathbf{R}^{n}, \quad\left|t-t_{0}\right| \leqslant \rho
$$

since

$$
\frac{d}{d t} \hat{Q}_{t_{0}, t} \varphi=-\left((\operatorname{grad} \varphi) \circ Q_{t_{0}, t}\right) \cdot \vec{X}_{t} Q_{t_{0}, t}=-\vec{X}_{t}\left(\varphi \circ Q_{t_{0}, t}\right)=-\vec{X}_{t} \circ \vec{Q}_{t_{0}, t} \varphi
$$

Hence we conclude by Proposition 1.1 that the family $\hat{P}_{t_{0, t}}$ is a left inverse of $\hat{Q}_{t_{0}, t}$. But $\hat{P}_{t_{0}, t}$ is also a right inverse of $\hat{Q}_{t_{0}, t}$, as follows at once from the remark following (1.29) which states that a right Volterra series in an arbitrary field $\vec{X}_{\tau}$ is identical to the left Volterra series in $-\vec{X}_{\tau}$ if we permute $t_{0}$ and $t$.

Thus the families $\hat{P}_{t_{0} t}$ and $\hat{Q}_{t_{0},},\left|t-t_{0}\right|<\rho$, are mutually inverse flows which satisfy (2.7) and (2.8), and which, by Proposition 1.1, are their unique solutions. According to Proposition 1.2, the flows $\hat{P}_{t_{0}, t}$ and $\hat{Q}_{t_{0}, t}$ can be defined for arbitrary $t_{0}$ and $t$ so that they are solutions of these equations and

$$
\hat{P}_{t_{0}, t}=\hat{P}_{t_{0}, t_{1}} \circ \hat{P}_{t_{1}, t_{2}} \circ \ldots \circ \hat{P}_{t_{m}, t} \quad \forall t_{1}, \ldots, t_{m} \in \mathbf{R}
$$

and similarly for $\hat{Q}_{t_{0}, t}$. By Proposition 2.1, if $\left|t_{j}-t_{j+1}\right|<\rho$, then $\hat{P}_{t_{0, t}}$ is representable as a composition of analytic diffeomorphisms, and therefore is an analytic diffeomorphism for all $t_{0}$ and $t$ in $\mathbf{R}$.

We call the analytic flows $\hat{P}_{t_{0}, t}$ and $\hat{Q}_{t_{0}, t}, t_{0}, t \in \mathbf{R}$, just constructed right and left chronological exponentials in $\vec{X}_{t}$ and in $-\vec{X}_{t}$, respectively, and we denote them by

$$
\begin{gathered}
\hat{P}_{t_{0}, t}=\left\langle\overrightarrow{\mathcal{V}}_{t_{0}, t}\left(\vec{X}_{\tau}\right) E\right\rangle=\overrightarrow{\exp } \int_{t_{0}}^{t} \vec{X}_{\tau} d \tau, \\
\hat{Q}_{t_{0}, t}=\left\langle\overleftarrow{V}_{t_{0}, t}\left(-\vec{X}_{\tau}\right) E\right\rangle=\overleftarrow{\exp } \int_{i_{0}}^{t}-\vec{X}_{\tau} d \tau
\end{gathered}
$$


(because of typographical considerations, we have put the expressions $\overrightarrow{\mathcal{V}}_{t_{0, t}}\left(\vec{X}_{\tau}\right) E$ and $\widetilde{\mho}_{t_{0}, t}\left(\vec{X}_{\tau}\right) E$ in angle brackets instead of putting a caret over them). If $\vec{X}_{t}$ is "commutative," i.e. if $\left[\vec{X}_{t^{\prime}}, \vec{X}_{t^{\prime \prime}}\right]=0$ for all $t^{\prime}, t^{\prime \prime} \in \mathbf{R}$, then (1.30) gives

$$
\overrightarrow{\exp } \int_{i_{0}}^{t} \vec{X}_{\tau} d \tau E=-\overline{\exp } \int_{t_{0}}^{t} \vec{X}_{\tau} d \tau E=\sum_{m=0}^{\infty} \frac{1}{m !}\left(\int_{t_{0}}^{t} \vec{X}_{\tau} d \tau\right)^{m} E=e^{\int_{t_{0}}^{t} \vec{X}_{\tau} d \tau}
$$

In conclusion we note that, in order to assure the existence of chronological exponentials, it is sufficient to require that $\vec{X}_{t}$ be locally integrable instead of uniformly integrable, as is clear from the proof. In this case the length of the interval of convergence with respect to $t_{0}$ and $t,\left|t-t_{0}\right| \leqslant \rho_{t_{0}}$, can approach zero as $t_{0} \rightarrow \infty$, but this does not interfere with the construction of flows for all $t_{0}$ and $t$ (see Proposition 1.2).

2. The case of an arbitrary field. If $\vec{X}_{t}$ is analytic, then, as was shown above, the construction of chronological exponentials is simple: we must apply the appropriate Volterra series to the identity mapping $E$ to get series of analytic mappings which converge to the unknown flows. For nonanalytic fields $\vec{X}_{t}$ the series $\vec{\vartheta}_{t_{0, t}}\left(\vec{X}_{\tau}\right) E$ and $\bar{\mho}_{t_{0}, t}\left(\vec{X}_{\tau}\right) E$ do not converge, and to obtain flows which give solutions of (2.7) and (2.8) we apply a more complicated procedure. We begin by estimating the seminorm $\left\|\hat{P}_{t_{0, t}} \varphi\right\|_{s, K}$ under the assumption that $\hat{P}_{t_{0}, t}$ satisfies (2.7).

Proposition 2.2. If $\hat{P}_{t_{0}, t}$ satisfies (2.7), where $\vec{X}_{t}, t \in \mathbf{R}$, is an arbitrarily (locally integrable) field, then for all $\varphi$ in $\Phi$, for $s \geqslant 0$, and for any compact set $K \subset \mathbf{R}^{n}$ we have $\left(t_{0} \leqslant t\right)$

$$
\begin{gathered}
\left\|\hat{P}_{t_{0}, t} \varphi\right\|_{s, K} \leqslant C_{1} e^{C_{2} \int_{t_{0}}^{t}\left\|X_{\tau}\right\|_{s} d \tau}\|\varphi\|_{s, M}, \\
C_{1}=(1+s)(2 n s)^{s}\left(1+n+\operatorname{diam} K+2 \int_{t_{0}}^{t}\left\|X_{\tau}\right\|_{0} d \tau\right)^{s}, \\
C_{2}=3 n(2 s+2)^{s+1} C_{1},
\end{gathered}
$$

where $M=O_{R}(K)$ is a neighborhood of radius $R=\int_{t_{0}}^{t}\left\|X_{\tau}\right\|_{0} d \tau$ of the compact set $K$.

ProOF. We put

$$
K_{t_{0}, t}=\bigcup_{\tau}\left\{P_{t_{0}, \tau}(K) \mid \tau \in\left[t_{0}, t\right]\right\}
$$

and introduce the quantity

$$
\begin{aligned}
N_{t_{0}, t} & =\sup _{\varphi \in \Phi}\left\{\frac{\left\|\hat{P}_{t_{0}, t} \varphi\right\|_{s, K}}{\|\varphi\|_{s+1, K_{t_{0}, t}}} \mid\|\varphi\|_{s+\mathbf{1}, K_{t_{0}, t}} \neq 0\right\} \\
& =\sup _{\boldsymbol{}}\left\{\left\|\hat{P}_{t_{0}, t} \varphi\right\|_{s, K} \mid\|\varphi\|_{s+\mathbf{1}, K_{t_{0}, t}}=1\right\}
\end{aligned}
$$

which, as is easy to see, is measurable in $t_{0}$ and $t$ and bounded on $\left[t_{0}, t\right]$. The measurability follows from the fact that the sup on the right-hand side need not be taken over all $\varphi \in \Phi$ but over only an arbitrary countable dense subset of $\Phi$; the boundedness

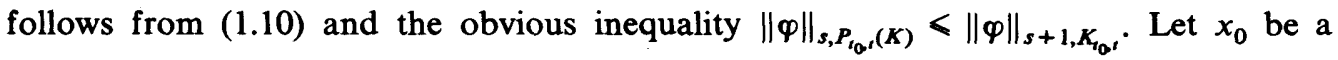


point at which

$$
\left\|\hat{P}_{t_{0}, t} \varphi\right\|_{s, K}=\sup _{x \in K} \sum_{\alpha=0}^{s} \frac{1}{\alpha !} \sup _{|h|=1}\left|\vec{h}^{\alpha}\left(\varphi \circ P_{t_{0}, t}\right)(x)\right|
$$

attains its upper bound, and let $m_{\varphi}$ be a polynomial of degree $\leqslant s$ whose derivatives of order up to and including $s$ at the point $p_{0}=P_{t_{0, t}}\left(x_{0}\right)$ coincide with the corresponding derivatives of $\varphi$ at the same point,

$$
\mathfrak{m}_{\varphi}(x)=\sum_{\alpha=0}^{s} \frac{1}{\alpha !_{i}}\left(\overrightarrow{x-p_{0}}\right) \varphi\left(p_{0}\right), \quad x \in \mathbf{R}^{n}
$$

In this case

$$
\begin{gathered}
\left\|\hat{P}_{t_{0}, t} \varphi\right\|_{s, K}=\sum_{\alpha \div-1}^{s} \frac{1}{\alpha !} \sup _{|h|=1}\left|\vec{h}^{\alpha}\left(\mathfrak{m}_{\varphi} \circ P_{t_{0}, t}\right)\left(x_{0}\right)\right| \leqslant\left\|\hat{P}_{t_{0}, t} \mathfrak{m}_{\varphi}\right\|_{s, K} ; \\
\left\|\mathfrak{m}_{\varphi}\right\|_{s, p_{0}} \leqslant\|\varphi\|_{s, K_{t_{0}, t}} .
\end{gathered}
$$

In the finite-dimensional space of all real polynomials of degree $<s$, all norms are equivalent, so there exists a constant $C_{1}$ which does not depend on the choice of the polynomial $m$ of degree $<s$ such that

$$
\frac{\|\mathrm{m}\|_{s, K_{t_{0}, t}}}{\|\mathrm{~m}\|_{s, p_{0}}} \leqslant C_{1}
$$

The inequalities (2.10) and (2.11) give the estimate

$$
\frac{\left\|P_{t_{0}, t} \varphi\right\|_{s, K}}{\|\varphi\|_{s, K_{t_{0}, t}}} \leqslant \frac{\left\|P_{t_{0}, t} \mathfrak{m}_{\varphi}\right\|_{s, K}}{\left\|m_{\varphi}\right\|_{s, p_{0}}} \leqslant C_{1} \frac{\left\|\hat{P}_{t_{0}, t} \mathrm{~m}_{\varphi}\right\|_{s, K}}{\left\|m_{\varphi}\right\|_{s, K_{t_{0}, t}}}=C_{1} \frac{\left\|P_{t_{0}, t} \mathfrak{m}_{\varphi,}\right\|_{s, K}}{\left\|\mathfrak{m}_{\varphi}\right\|_{s+1, K_{t_{0}, t}}} \leqslant C_{1} N_{t_{0}, t} .
$$

We show that we can take

$$
C_{1}=(1+s)(2 n s)^{s}\left(1+n+\operatorname{diam} K_{t_{0}, t}\right)^{s} .
$$

From the estimate

$$
\left|\vec{h}_{1} \circ \cdots \circ \vec{h}_{k} \frac{1}{\left(w^{1}-z^{1}\right) \cdots\left(w^{n}-z^{n}\right)}\right| \leqslant \frac{k !}{\sigma^{n}}\left(\frac{n}{\sigma}\right)^{k}, \quad\left|h_{j}\right|=1, \quad\left|w^{\alpha}-z^{\alpha}\right| \geqslant \sigma,
$$

it follows that for $\sigma=n\left(S_{\alpha}\right.$ is a circle of radius $n$ with center $\left.z^{\alpha}\right)$

$$
\begin{gathered}
\sup _{x \in K_{t_{0}, t}}\left|\vec{h}_{1} \circ \cdots \circ \vec{h}_{k} \mathfrak{m}(x)\right| \\
=\frac{1}{(2 \pi)^{n}} \sup _{z \in K_{t_{0}, t}}\left|\vec{h}_{1} \circ \ldots \circ \vec{h}_{k} \int_{S_{1}} d w^{1} \ldots \int_{S_{n}} d w^{n} \frac{\mathfrak{m}(w)}{\left(w^{1}-z^{1}\right) \cdots\left(w^{n}-z^{n}\right)}\right| \\
\leqslant \frac{1}{(2 \pi)^{n}} \frac{k !}{\sigma^{n}}\left(\frac{n}{\sigma}\right)^{k}(2 \pi n)^{n}\|\mathfrak{m} \mid\|_{V_{n}}^{n}\left(K_{t_{0}, t}\right)=k !\|\mathfrak{m}\|_{V_{n}}^{C^{n}}\left(K_{t_{0}, t}\right),
\end{gathered}
$$

where

$$
\|\mathfrak{m}\| V_{V_{n}\left(K_{t_{0}, t}\right)}^{C^{n}}=\sup _{w \in V_{n}\left(K_{t_{0}, t}\right)}|\mathfrak{m}(w)|
$$


and $V_{n}\left(K_{t_{0}, t}\right)$ is a complex neighborhood of radius $n$ of the compact set $K_{t_{0}, t}$. Therefore

$$
\begin{aligned}
& \|\mathfrak{m}\|_{s, K_{t_{0}, t}}=\sup _{x \in K_{t_{0}, t}} \sum_{\alpha=0}^{s} \frac{1}{\alpha !} \sup _{|h|=1}\left|\vec{h}^{\alpha} \mathfrak{m}(x)\right| \\
& \leqslant \sum_{\alpha=0}^{s} \frac{1}{\alpha !} \alpha !\|\mathfrak{m}\|_{V_{n}\left(K_{t_{0}, t}\right)}^{\mathbf{C}^{n}}=(1+s)\|\mathfrak{m}\|_{V_{n}\left(K_{t_{0}, t}\right)}^{\mathbf{C}^{n}} .
\end{aligned}
$$

\section{Consequently}

$$
\begin{aligned}
& \|\mathfrak{m}\|_{s, K_{t_{0}, t}} \leqslant(1+s)\|\mathfrak{m}\|_{V_{n}\left(K_{t_{0}, t}\right)}^{\mathbb{C}^{n}}=(1+s) \sup _{z \in V_{n}\left(K_{t_{0}, t}\right)}\left|\sum_{\alpha=0}^{s} \frac{1}{\alpha !}\left(\overrightarrow{z-p_{0}}\right) \mathfrak{m}\left(p_{0}\right)\right| \\
& \leqslant(s+1) \sup _{z \in V_{n}\left(K_{\left.t_{0}, t\right)}\right)}\left(1+\left|z-p_{0}\right|\right)^{s} \sup _{z \in V_{n}\left(K_{t_{0}, t}\right)}\left|\sum_{\alpha==0}^{s} \frac{1}{\alpha !} \overline{\left(\frac{z-p_{0}}{\left|z-p_{0}\right|}\right)} \mathfrak{m}\left(p_{0}\right)\right| .
\end{aligned}
$$

(The symbol

$$
\overrightarrow{z-p_{0}}=\sum_{\alpha=1}^{n}\left(z^{\alpha}-p_{0}^{\alpha}\right) \partial_{\alpha}
$$

denotes the differential operator with (constant) complex coefficients $z^{\alpha}-p_{0}^{\alpha}$.)

It is easy to see that

$$
\sup _{z \in V_{n}\left(K_{t_{0}, t}\right)}\left|\sum_{\alpha=0}^{s} \frac{1}{\alpha !} \overline{\left(\frac{z-p_{0}}{\left|z-p_{0}\right|}\right)} \mathfrak{m}\left(p_{0}\right)\right| \leqslant(2 n s)^{s}\|\mathfrak{m}\|_{s, p_{0}} .
$$

In fact, if none of the coordinates $w^{k}, k=1, \ldots, n$, of the complex vector $w$ exceed one in modulus, then, using (1.8), we would get

$$
\begin{aligned}
\left|\sum_{\alpha=0}^{s} \frac{1}{\alpha !}(w)^{\alpha} \mathfrak{m}\left(p_{0}\right)\right| & \leqslant \sum_{\alpha=0}^{s} \frac{1}{\alpha !} \sum_{k_{1}, \ldots, k}^{n}|| w^{k_{1}} \ldots w^{k_{\alpha}}\left|\partial_{k_{1}} \circ \ldots \circ \partial_{k_{\alpha}} \mathfrak{m}\left(p_{0}\right)\right| \\
& \leqslant \sum_{\alpha=0}^{s} \frac{1}{\alpha !} n^{\alpha}(2 \alpha)^{\alpha}\|\mathfrak{m}\|_{s, p_{0}} \leqslant(2 n s)^{s}\|\mathfrak{m}\|_{s, p_{0}} .
\end{aligned}
$$

Combining this with (2.14), we arrive at (2.13).

Equation (2.7), together with (2.12) and (1.9), allow us to write

$$
\begin{gathered}
\left\|\hat{P}_{t_{0}, t} \varphi\right\|_{s, K} \leqslant\|\varphi\|_{s, K}+\int_{t_{0}}^{t}\left\|\hat{P}_{t_{0}, \tau} \circ \vec{X}_{\tau} \varphi\right\|_{s, K} d \tau \\
\leqslant\|\varphi\|_{s, K}+3 n(2 s+2)^{s+1} C_{1} \int_{t_{0}}^{t} N_{t_{0}, \tau}\left\|X_{\tau}\right\|_{s} d \tau\|\varphi\|_{s+1, K_{t_{0}, t}} .
\end{gathered}
$$

Dividing by $\|\varphi\|_{s+1, K_{t_{0},}}$, we arrive at

$$
\frac{\left\|\hat{P}_{t_{0, t}} \varphi\right\|_{s, K}}{\|\varphi\|_{s+1, K_{t_{0}, t}}} \leqslant 1+3 n(2 s+2)^{s+1} C_{1} \int_{t_{0}}^{t} N_{t_{0}, \tau}\left\|X_{\tau}\right\|_{s} d \tau .
$$

Since the right-hand side does not depend on $\varphi$, we get

$$
N_{t_{0}, t} \equiv 1+C_{2} \int_{t_{0}}^{t} N_{t_{0}, \tau}\left\|X_{\tau}\right\|_{s} d \tau, \quad C_{2}=3 n(2 s+2)^{s+1} C_{1},
$$


from which it follows by Gronwall's lemma that

$$
N_{t_{0}, t} \leqslant e^{C_{2} \int_{t_{0}}^{t}\left\|X_{\tau}\right\|_{s} d \tau}
$$

by virtue of the boundedness of $N_{t_{0}, t}$, or, by (2.12),

$$
\left\|\hat{P}_{t_{0}, t} \varphi\right\|_{s, K} \leqslant C_{1} e^{C_{2} \int_{t_{0}}^{t}\left\|X_{\tau}\right\|_{s} d \tau}\|\varphi\|_{s, K_{t_{0}, t}} .
$$

It remains to estimate the set $K_{t_{0}, t}$ in terms of $K$ and $\vec{X}_{t}$. We have

$$
\begin{aligned}
\operatorname{diam} K_{t_{0}, t}= & \max _{\substack{x, y \in K \\
\tau^{\prime}, \tau^{\prime \prime} \in\left[t_{0}, t\right]}}\left|x+\int_{t_{0}}^{\tau^{\prime}} X_{\theta} \circ P_{t_{0}, \theta}(x) d \theta-\left(y+\int_{t_{0}}^{\tau^{\prime \prime}} X_{\theta} \circ P_{t_{0}, \theta}(y) d \theta\right)\right| \\
& \approx \operatorname{diam} K+2 \int_{t_{0}}^{t}\left\|X_{\tau}\right\|_{0} d \tau, \quad K_{t_{0}, t} \subset \int_{t_{0}}^{O} \| X_{\tau} \psi_{i, 0} d \tau
\end{aligned}
$$

which concludes the proof of the proposition.

We assume now that the bounded (locally integrable) field $\vec{X}_{t}$ is given, i.e., that it satisfies $\left\|X_{t}\right\|_{s}<\infty$ for all $s>0$. We consider the entire function of $z \in \mathbf{C}^{n}$

$$
\delta_{\varepsilon}(z)=\frac{1}{(\varepsilon \sqrt{\pi})^{n}} e^{-\left(\frac{z}{\varepsilon}\right)^{2}}, \quad z^{2}=\sum_{\alpha=1}^{n}\left(z^{\alpha}\right)^{2},
$$

which depends on the parameter $\varepsilon>0$. Taking its convolution with $X_{t}$, we get the entire function of $z$

$$
X_{t}^{\varepsilon}(z)=\delta_{\varepsilon} * X_{t}(z)=\frac{1}{(\varepsilon \sqrt{\pi})^{n}} \int_{\mathbf{R}^{n}} e^{-\left(\frac{z-x}{\varepsilon}\right)^{2}} X_{t}(x) d x .
$$

Considered on $\mathbf{R}^{n}$, for each fixed $\varepsilon>0$ it belongs to the space $\Omega_{\sigma}^{n}$ for all $\sigma>0$. For real values of the argument, $X_{t}^{\varepsilon}$ can be calculated from

$$
X_{t}^{\varepsilon}(x)=\frac{1}{(\varepsilon \sqrt{\pi})^{n}} \int_{\mathbf{R}^{n}} e^{-\left(\frac{x-y}{\varepsilon}\right)^{2}} X_{t}(y) d y=\frac{1}{\sqrt{\pi^{n}}} \int_{\mathbf{R}^{n}} e^{-y^{2}} X_{t}(x-\varepsilon y) d y
$$

consequently

$$
\begin{gathered}
\left\|X_{t}^{\varepsilon}\right\|_{s}=\sup _{x \in \mathbb{R}^{n}} \sum_{\alpha=0}^{s} \frac{1}{\alpha !} \sup _{|h|=1} \frac{1}{\sqrt{\pi^{n}}}\left|\int_{\mathbb{R}^{n}} e^{-y^{2} \vec{h}^{\alpha}} X_{t}(x-\varepsilon y) d y\right| \\
\leqslant \frac{1}{V \bar{\pi}^{n}} \int_{\mathbf{R}^{n}} e^{-y^{2}} d y \sup _{x \in \mathbb{R}^{n}} \sum_{\alpha=0}^{s} \frac{1}{\alpha !} \sup _{|h|=1}\left|\vec{h}^{\alpha} X_{t}(x)\right|=\left\|X_{t}\right\|_{s} .
\end{gathered}
$$

Furthermore, the identity

$$
\begin{aligned}
\left(X_{t}^{\mathbf{e}^{\prime}}-X_{t}^{\varepsilon^{\prime \prime}}\right)(x)=\frac{1}{\sqrt{\pi^{n}}} \int_{\mathbf{R}^{n}} d y e^{-y^{2}}\left(X_{t}\left(x-\varepsilon^{\prime} y\right)-X_{t}\left(x-\varepsilon^{\prime \prime} y\right)\right) \\
=\frac{\varepsilon^{\prime \prime}-\varepsilon^{\prime}}{\sqrt{\bar{\pi}^{n}}} \int_{\mathbf{R}^{n}} d y e^{-y^{2}} \int_{0}^{1} d \tau \operatorname{grad} X_{t}\left(x-\varepsilon^{\prime \prime} y+\tau\left(\varepsilon^{\prime \prime}-\varepsilon^{\prime}\right) y\right) \cdot y
\end{aligned}
$$


together with (1.9) gives that

$$
\begin{gathered}
\left\|X_{t}^{\varepsilon^{\prime}}-X_{t}^{\varepsilon^{\prime \prime}}\right\|_{s} \leqslant\left|\varepsilon^{\prime}-\varepsilon^{\prime \prime}\right| C_{3}\left\|X_{t}\right\|_{s+1}, \\
C_{3}=3 n(2 s+2)^{s+1} \frac{1}{\sqrt{\pi^{n}}} \int_{\mathbf{R}^{n}}|y| e^{-y^{2}} d y .
\end{gathered}
$$

In subsection 1 we proved the existence of mutually inverse analytic flows

$$
\hat{P}_{t_{0}, t}^{\varepsilon}=\overrightarrow{\exp } \int_{t_{0}}^{t} \vec{X}_{\tau}^{\varepsilon} d \tau, \quad \hat{Q}_{t_{0}, t}^{\varepsilon}=\overleftarrow{\exp } \int_{t_{0}}^{t}-\vec{X}_{\tau}^{\varepsilon} d \tau, \quad t_{0}, t \in \mathbf{R},
$$

which satisfy

$$
\hat{P}_{t_{0}, t}^{\varepsilon}=\mathrm{Id}+\int_{t_{0}}^{t} \hat{P}_{t_{0}, \tau}^{\varepsilon} \circ \vec{X}_{\tau}^{\varepsilon} d \tau, \quad \hat{Q}_{t_{0}, t}^{\varepsilon}=\mathrm{Id}-\int_{t_{0}}^{t} \vec{X}_{\tau}^{\varepsilon} \circ \hat{Q}_{t_{0}, \tau}^{\varepsilon} d \tau .
$$

We show that as $\varepsilon \rightarrow 0$ the flows $P_{t_{0}, t}^{e}$ and $Q_{t_{0}, t}^{e}$ converge in the topology of $\Phi^{n}$ to the mutually inverse flows $P_{t_{0}, t}$ and $Q_{t_{0}, t}, t_{0}, t \in \mathbf{R}$, where $\hat{P}_{t_{0}, t}$ and $\hat{Q}_{t_{0}, t}$ satisfy (2.7) and (2.8).

From

$$
\hat{P}_{t_{0}, t}^{\varepsilon^{\prime}}-\hat{P}_{t_{0}, t}^{\varepsilon^{\prime \prime}}=\int_{t_{0}}^{t} \hat{P}_{t_{0}, \tau}^{\varepsilon^{\prime}} \circ\left(\vec{X}_{\tau}^{\varepsilon^{\prime}}-\vec{X}_{\tau}^{\varepsilon^{\prime \prime}}\right) d \tau+\int_{t_{0}}^{t}\left(\hat{P}_{t_{0}, \tau}^{\varepsilon^{\prime}}-\hat{P}_{t_{0}, \tau}^{\varepsilon^{\prime \prime}}\right) \circ \vec{X}_{\tau}^{\varepsilon^{\prime \prime}} d \tau .
$$

(2.16), (2.17), (1.9), (1.10), and (1.11) we get

$$
\begin{gathered}
\left\|P_{t_{0}, t}^{\varepsilon^{\prime}}-P_{t_{0}, t}^{\varepsilon^{\prime \prime}}\right\|_{s, K} \leqslant\left(1+9 n^{2}(2 s)^{2 s}\right)^{s} C_{3}\left|\varepsilon^{\prime}-\varepsilon^{\prime \prime}\right| \int_{t_{0}}^{t}\left(1+\left\|P_{t_{0}, \tau}^{\varepsilon^{\prime}}\right\|_{s, K}\right)^{s}\left\|X_{\tau}\right\|_{s} d \tau \\
+3 n(2 s+2)^{s+1}\left(1+9 n^{2}(2 s)^{2 s}\right)^{s} \int_{t_{0}}^{t}\left(1+\left\|P_{t_{0}, \tau}^{\varepsilon^{\prime}}\right\|_{s, K}+\left\|P_{t_{0}, \tau}^{\varepsilon^{\prime \prime}}\right\|_{s, K}\right)^{s} \\
\times\left\|X_{\tau}\right\|_{s+1}\left\|P_{t_{0}, \tau}^{\varepsilon^{\prime}}-P_{t_{0}, \tau}^{\varepsilon^{\prime \prime}}\right\|_{s, K} d \tau .
\end{gathered}
$$

The quantities $\left\|P_{t_{0}, \tau}^{e^{\prime}}\right\|_{s, K}$ and $\left\|P_{t_{0}, \tau}^{e^{\prime \prime}}\right\|_{s, K}$ in this estimate are majorized by a locally integrable function which does not depend on $\varepsilon$, by virtue of (2.16) and Proposition 2.2. Therefore applying Gronwall's lemma to it, we get that

$$
\lim _{\mathbf{\varepsilon}^{\prime}, \varepsilon^{\prime \prime} \rightarrow 0}\left\|P_{t_{0}, t}^{\varepsilon^{\prime}}-P_{t_{0}, t}^{\varepsilon^{*}}\right\|_{s, K}=0
$$

The convergence for $Q_{t_{0}, t}^{e}$ can be proved similarly, or it follows from the convergence just proved for $P_{t_{0}, t}^{e}$ and the fact that the left flow $Q_{t_{0, t}}^{e}$ is also a right flow:

$$
Q_{t_{0}, t}^{\varepsilon}=\overleftarrow{\mho}_{t_{0}, t}\left(-\vec{X}_{\tau}^{\varepsilon}\right) E=\overrightarrow{\widetilde{V}}_{t, t_{\bullet}}\left(\vec{X}_{\tau}^{\mathrm{e}}\right) E
$$

The limit transformations $P_{t_{0}, t}$ and $Q_{t_{0}, t}$ are mutually inverse diffeomorphisms for all $t_{0}$, $t \in \mathbf{R}$, since it is easy to get from (1.11) that

$$
P_{t_{0}, t} \circ Q_{t_{0}, t}=Q_{t_{0}, t} \circ P_{t_{0}, t}=\lim _{\varepsilon \rightarrow 0} P_{t_{0}, t}^{\boldsymbol{e}} \circ Q_{t_{0}, t}^{\mathcal{E}}=E .
$$

Finally, it is obvious that $\hat{P}_{t_{0}, t}$ and $\hat{Q}_{t_{0} t}$ satisfy (2.7) and (2.8) since the limit flows satisfy them. 
Thus we have constructed flows $\hat{P}_{t_{0}, t}$ and $\hat{Q}_{t_{0}, t}, t_{0}, t \in \mathbf{R}$, which satisfy (2.7) and (2.8); we call them right and left chronological exponentials in $\vec{X}_{t}$ and $-\vec{X}_{t}$, respectively, and we write

$$
\begin{gathered}
\hat{P}_{t_{0}, t}=\overrightarrow{\exp } \int_{t_{0}}^{t} \vec{X}_{\tau} d \tau=\mathrm{Id}+\int_{t_{0}}^{t}\left(\overrightarrow{\exp } \int_{t_{0}}^{\tau} \vec{X}_{\theta} d \theta\right) \vec{X}_{\tau} d \tau \\
\hat{Q}_{t_{0}, t}=\overleftarrow{\exp } \int_{t_{0}}^{t}-\vec{X}_{\tau} d \tau=\mathrm{Id}-\int_{t_{0}}^{t} \vec{X}_{\tau} \circ\left(\widetilde{\exp } \int_{t_{0}}^{\tau}-\vec{X}_{\theta} d \theta\right) d \tau \\
\overrightarrow{\exp } \int_{t_{0}}^{t} \vec{X}_{\tau} d \tau \circ \stackrel{\leftarrow-\exp }{t} \int_{t_{0}}^{t}-\vec{X}_{\tau} d \tau=\overleftarrow{\exp } \int_{t_{0}}^{t}-\vec{X}_{\tau} d \tau \circ \overrightarrow{\exp } \int_{t_{0}}^{t} \vec{X}_{\tau} d \tau=\mathrm{Id}
\end{gathered}
$$

For arbitrary $t_{1}, \ldots, t_{m}$ we have

$$
\overrightarrow{\exp } \int_{t_{0}}^{t} \vec{X}_{\tau} d \tau=\overrightarrow{\exp } \int_{t_{0}}^{t_{1}} \vec{X}_{\tau} d \tau \circ \overrightarrow{\exp } \int_{t_{1}}^{t_{2}} \vec{X}_{\tau} d \tau \circ \ldots \overrightarrow{\exp } \int_{t_{m}}^{t} \vec{X}_{\tau} d \tau
$$

in particular,

$$
\overrightarrow{\exp } \int_{t_{0}}^{t} \vec{X}_{\tau} d \tau=\left(\overrightarrow{\exp } \int_{i}^{t_{0}} \vec{X}_{\tau} d \tau\right)^{-1}=\overleftarrow{\exp } \int_{t}^{t_{0}}-\vec{X}_{\tau} d \tau
$$

In subsection 1 , we showed that for all $t_{0}$ it is possible to find $\rho_{t_{0}}>0$ such that for $\left|t-t_{0}\right|<\rho_{t_{0}}$ the series

$$
\begin{gathered}
\overrightarrow{\mathfrak{V}}_{t_{0}, t}\left(\vec{X}_{\tau}^{\varepsilon}\right) E=\left(\mathrm{Id}+\sum_{\alpha=1}^{\infty} \int_{t_{0}}^{t} d \tau_{1} \int_{t_{0}}^{\tau_{2}} d \tau_{2} \ldots \int_{t_{0}}^{\tau_{\alpha-1}} d \tau_{\alpha} \vec{X}_{\tau_{\alpha}}^{\varepsilon} \cdots \circ \vec{X}_{\tau_{1}}^{\varepsilon}\right) E, \\
\vec{X}_{t}^{\varepsilon}={\overrightarrow{\delta_{\varepsilon}} * \vec{X}_{t}}
\end{gathered}
$$

converges; we denote the corresponding operator flow by putting the expression under consideration in angle brackets. If $t_{0}$ and $t$ are arbitrary, and if we insert points $t_{0}<t_{1}<\cdots<t_{m}=t$ between them such that $\left|t_{j}-t_{j+1}\right|<\rho_{t}$, we can write

$$
\begin{gathered}
\overrightarrow{\exp } \int_{t_{0}}^{t} \vec{X}_{\tau} d \tau=\lim _{\varepsilon \rightarrow 0}\left\langle\overrightarrow{\mathfrak{V}}_{t_{0}, t_{1}}\left(\vec{X}_{\tau}^{\varepsilon}\right) E\right\rangle \circ\left\langle\overrightarrow{\mathcal{V}}_{t_{1}, t_{2}}\left(\vec{X}_{\tau}^{\varepsilon}\right) E\right\rangle \circ \ldots \circ\left\langle\overrightarrow{\mathfrak{V}}_{t_{m-1}, t}\left(\vec{X}_{\tau}^{\varepsilon}\right) E\right\rangle, \\
\vec{X}_{t}^{\varepsilon}={\overrightarrow{\delta_{\varepsilon} * X_{t}}} .
\end{gathered}
$$

We call the expression on the right-hand side the operation of "exponential summation" of the Volterra series $\vec{V}_{t_{0, t}}\left(\vec{X}_{\tau}\right)$. Even in the case where $\vec{X}_{t}$ is analytic, the Volterra series does not converge in the topology of simple convergence in $\mathcal{L}(\Phi)$ in the usual sense, so we write

$$
\overrightarrow{\exp } \int_{t_{0}}^{t} \vec{X}_{\tau} d \tau \cong \mathrm{Id}+\sum_{m=1}^{\infty} \int_{t_{0}}^{t} d \tau_{1} \int_{t_{0}}^{\tau_{1}} d \tau_{2} \ldots \int_{t_{0}}^{\tau_{m-1}} d \tau_{m} \vec{X}_{\tau_{m}} \ldots \circ \dddot{X}_{\tau_{1}}
$$

understanding by the "asymptotic equality" the relation which is defined precisely in the following proposition. 
Proposition 2.3. Let $\vec{X}_{t}, t \in \mathbf{R}$, be a bounded (locally integrable) field and $\hat{P}_{t_{0}, t}$ the flow generated by it. Then for all $\varphi$ in $\Phi$ and $m>2$

$$
\begin{aligned}
& \left\|\left(\hat{P}_{t_{0}, t}-\left(\mathrm{Id}+\sum_{\alpha=1}^{m-1} \int_{t_{0}}^{t} d \tau_{1} \int_{t_{0}}^{\tau_{1}} d \tau_{2} \ldots \int_{t_{0}}^{\tau_{\alpha-1}} d \tau_{\alpha} \vec{X}_{\tau_{\alpha}} \circ \circ \circ \vec{X}_{\tau_{1}}\right)\right) \varphi\right\|_{s, K} \\
& \leqslant\left. C_{1} e^{C_{2} \mid \int_{t_{0}}^{t}\left\|X_{\tau}\right\|_{s} d \tau}\right|_{\left(3 n(2 s+2 m)^{s+m}\right)^{m}} ^{m} \frac{1}{m !}\left|\int_{t_{0}}^{t}\left\|X_{\tau}\right\|_{s+m-1} d \tau\right|\|\varphi\|_{s+m, M}^{m},
\end{aligned}
$$

where $C_{1}, C_{2}$, and $M$ are defined in Proposition 2.2.

Proof. Using Proposition 2.2 and (1.9), we can write

$$
\begin{aligned}
& \left\|\hat{P}_{t_{0}, \tau_{m}} \circ \vec{X}_{\tau_{m}} \circ \ldots \circ \vec{X}_{\tau_{1}} \varphi\right\|_{s, K}
\end{aligned}
$$

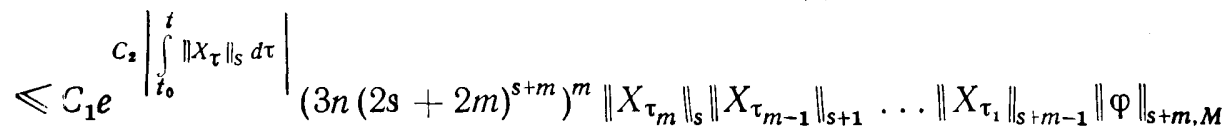

$$
\begin{aligned}
& \leqslant C_{1} e^{C_{2}\left|\int_{t_{0}}^{t}\left\|X_{\tau}\right\|_{s} d \tau\right|} \mid\left(3 n(2 s+2 m)^{s+m}\right)^{m}\left\|X_{\tau_{m}}\right\|_{s+m-1} \cdots\left\|X_{\tau_{1}}\right\|_{s+m-1}\|\varphi\|_{s+m, M},
\end{aligned}
$$

which proves the proposition if we apply the obvious identities

$$
\begin{gathered}
\hat{P}_{t_{0}, t}-\left(\mathrm{Id}+\sum_{\alpha=1}^{m-1} \int_{t_{0}}^{t} d \tau_{1} \int_{t_{0}}^{\tau_{1}} d \tau_{2} \ldots \int_{i_{0}}^{\tau_{\alpha-1}} d \tau_{\alpha} \vec{X}_{\tau_{\alpha}} \circ \ldots \circ \bar{X}_{\tau_{1}}\right) \\
=\int_{i_{0}}^{t} d \tau_{1} \int_{t_{0}}^{\tau_{1}} d \tau_{2} \ldots \int_{t_{0}}^{\tau_{m-1}} d \tau_{m} \hat{P}_{t_{0}, \tau_{m}} \circ \bar{X}_{\tau_{m}} \circ \ldots \circ \bar{X}_{\tau_{1}}, \\
\int_{t_{0}}^{t} d \tau_{1} \int_{t_{0}}^{\tau_{1}} d \tau_{2} \ldots \int_{t_{0}}^{\tau_{m-1}} d \tau_{m}\left\|X_{\tau_{m}}\right\|_{s+m-1} \cdots\left\|X_{\tau_{1}}\right\|_{s+m-1}=\frac{1}{m !}\left(\int_{i_{0}}^{t}\left\|X_{\tau}\right\|_{s+m-1} d \tau\right)^{m} .
\end{gathered}
$$

\section{§3. Representation of perturbation flows}

(the "variation of constants" formula)

Two concepts in the theory of ordinary differential equations which have basic significance for us are the perturbation flow of a given flow

$$
\hat{P}_{t_{0}, t}=\overrightarrow{\exp } \int_{t_{0}}^{t} \vec{X}_{\tau} d \tau
$$

and the variation of a flow $\hat{P}_{t_{0}, t}$. In this section, we obtain formulas which represent a perturbation flow in terms of input data, and the following two sections are devoted to a study of the variation of a given flow.

1. Representation of a perturbation flow. We will assume that the field $\vec{X}_{t}$ and the flow (3.1) are fixed. We will call an arbitrary field $\vec{Y}_{t}$ a perturbation of $\vec{X}_{t}$, and the flow $\overrightarrow{\exp } \int_{t_{0}}^{t}\left(\vec{X}_{\tau}+\vec{Y}_{\tau}\right) d \tau$ the corresponding perturbed flow. 
We consider the problem: find flows $\hat{C}_{t_{0, t}}\left(\vec{Y}_{\tau}\right)$ and $\hat{D}_{t_{0, t}}\left(\vec{Y}_{\tau}\right)$ which satisfy

$$
\begin{aligned}
& \overrightarrow{\exp } \int_{t_{0}}^{t}\left(\vec{X}_{\tau}+\vec{Y}_{\tau}\right) d \tau=\hat{C}_{t_{0}, t}\left(\vec{Y}_{\tau}\right) \circ \overrightarrow{\exp } \int_{t_{0}}^{t} \vec{X}_{\tau} d \tau, \\
& \overrightarrow{\exp } \int_{t_{0}}^{t}\left(\vec{X}_{\tau}+\vec{Y}_{\tau}\right) d \tau=\overrightarrow{\exp } \int_{t_{0}}^{t} \vec{X}_{\tau} d \tau \circ \hat{D}_{t_{0}, t}\left(\vec{Y}_{\tau}\right) .
\end{aligned}
$$

We call $\hat{C}_{t_{0}, t}\left(\vec{Y}_{\tau}\right)$ a left perturbation flow for $\hat{P}_{t_{0}, t}$ for the perturbation field $\vec{Y}_{t}$, and $\hat{D}_{t_{0, t}}\left(\vec{Y}_{\tau}\right)$ a right perturbation flow.

We obtain a solution of the above-stated problem by the method of "variation of constants."

To this end, we write by (1.12)

$$
\left(\operatorname{Ad} \overrightarrow{\exp } \int_{t_{0}}^{t} \vec{X}_{\tau} d \tau\right) \vec{Z}=\hat{P}_{t_{0}, t} \circ \vec{Z} \circ \hat{P}_{t_{0}, t}^{-1} \in \operatorname{Der}(\Phi) \quad \forall \vec{Z} \in \operatorname{Der}(\Phi),
$$

regarding Ad $\hat{P}_{t_{0} t}$ as a family of linear transformations of $\operatorname{Der}(\Phi)$ into itself which depends on $t_{0}, t \in \mathbf{R}$. Differentiation with respect to $t$ gives

$$
\frac{d}{d t}\left(\operatorname{Ad} \hat{P}_{t_{0}, t}\right) \vec{Z}=\hat{P}_{t_{0}, t} \circ\left(\vec{X}_{t} \circ \vec{Z}-\vec{Z} \circ \vec{X}_{t}\right) \circ \hat{P}_{t_{0}, t}^{-1}=\left(\operatorname{Ad} \hat{P}_{t_{0}, t} \circ \operatorname{ad} \vec{X}_{t}\right) \vec{Z}
$$

which, by virtue of the arbitrariness of $\vec{Z} \in \operatorname{Der}(\Phi)$, may be considered, purely formally, as a linear equation for $\mathrm{Ad} \hat{P}_{t_{0}, t}$ :

$$
\frac{d}{d t} \operatorname{Ad} \overrightarrow{\exp } \int_{t_{0}}^{t} \vec{X}_{\tau} d \tau=\operatorname{Ad} \overrightarrow{\exp } \int_{t_{0}}^{t} \vec{X}_{\tau} d \tau \circ \operatorname{ad} \vec{X}_{t}
$$

This equation gives a purely formal reason for calling Ad $\overrightarrow{\exp } \int_{t_{0}}^{t} \vec{X}_{\tau} d \tau$ a "right chronological exponential in ad $\vec{X}_{t}^{\prime \prime}$ :

$$
\operatorname{Ad} \overrightarrow{\exp } \int_{t_{0}}^{t} \vec{X}_{\tau} d \tau=\overrightarrow{\exp } \int_{t_{0}}^{t} \operatorname{ad} \vec{X}_{\tau} d \tau
$$

We note several simple properties of Ad $\hat{P}_{t_{0}, t}$ which are completely consistent with the notation of (3.4) and (3.5). First of all, it is clear that Ad $\hat{P}_{t_{00} t}$ is invertible, and

$$
\left(\operatorname{Ad} \hat{P}_{t_{0}, t}\right)^{-1}=\operatorname{Ad} \hat{P}_{t_{0}, t}^{-1}
$$

and correspondingly

$$
\begin{gathered}
\frac{d}{d t}\left(\operatorname{Ad} \hat{P}_{t_{0}, t}\right)^{-1} \vec{Z}=-\operatorname{ad} \vec{X}_{t} \circ\left(\operatorname{Ad} \hat{P}_{t_{0}, t}\right)^{-1} \vec{Z} \\
\left(\operatorname{Ad} \hat{P}_{t_{0}, t}\right)^{-1}=\operatorname{Ad} \overleftarrow{\exp } \int_{t_{0}}^{t}-\vec{X}_{\tau} d \tau=\overleftarrow{\exp } \int_{t_{0}}^{t}-\operatorname{ad} \vec{X}_{\tau} d \tau
\end{gathered}
$$

Furthermore, we have

$$
\operatorname{Ad} \hat{P}_{t_{0}, t_{2}} \circ \operatorname{Ad} \hat{P}_{t_{1}, t_{2}} \circ \cdots \circ \operatorname{Ad} \hat{P}_{t_{m^{\prime}, t}}=\operatorname{Ad} \hat{P}_{t_{0}, t}
$$


in particular,

$$
\left(\operatorname{Ad} \hat{P}_{t_{0}, t}\right)^{-1}=\operatorname{Ad} \hat{P}_{t, t_{0}}=\overrightarrow{\exp } \int_{t}^{t_{0}} \operatorname{ad} \vec{X}_{\tau} d \tau .
$$

We define the action of Ad $\hat{P}_{t_{1}, t_{2}}$ on flows by

$$
\operatorname{Ad} \hat{P}_{t_{1}, t_{2}} \overrightarrow{\exp } \int_{t_{0}}^{t} \vec{Y}_{\tau} d \tau=\hat{P}_{t_{1}, t_{2}} \circ \overrightarrow{\exp } \int_{t_{0}}^{t} \vec{Y}_{\tau} d \tau \circ \hat{P}_{t_{1}, t_{2}}^{-1}
$$

Then we have

$$
\operatorname{Ad} \hat{P}_{t_{1}, t_{2}} \overrightarrow{\exp } \int_{t_{0}}^{t} \vec{Y}_{\tau} d \tau=\overrightarrow{\exp } \int_{t_{0}}^{t} \operatorname{Ad} \hat{P}_{t_{1}, t_{2}} \vec{Y}_{\tau} d \tau \quad \forall t_{1}, t_{2} \in \mathbf{R}
$$

since if we differentiate the flow on the left-hand side with respect to $t$, we see that it satisfies

$$
\frac{d}{d t} \operatorname{Ad} \hat{P}_{t_{1}, t_{2}} \overrightarrow{\exp } \int_{t_{0}}^{t} \vec{Y}_{\tau} d \tau=\operatorname{Ad} \hat{P}_{t_{1}, t_{2}} \overrightarrow{\exp } \int_{t_{0}}^{t} \vec{Y}_{\tau} d \tau \circ \operatorname{Ad} \hat{P}_{t_{1,2} t} \vec{Y}_{t}
$$

Finally we note that if $\vec{Z}$ commutes with $\vec{X}_{t}$ for all $t$ in $\mathbf{R}$ (i.e. if ad $\vec{X}, \vec{Z}=0$ for all $t$ in R), then

$$
\operatorname{Ad} \overrightarrow{\exp } \int_{t_{0}}^{t} \vec{X}_{\tau} d \tau \vec{Z}=\vec{Z} \quad \forall t_{0}, t \in \mathbf{R}
$$

since

$$
\frac{d}{d t} \operatorname{Ad} \overrightarrow{\exp } \int_{t_{0}}^{t} \vec{X}_{\tau} d \tau \vec{Z}=\left(\operatorname{Ad} \overrightarrow{\exp } \int_{t_{0}}^{t} \vec{X}_{\tau} d \tau \circ \operatorname{ad} \vec{X} t\right) \vec{Z}=0
$$

We now express the left perturbation flow $\hat{C}_{t_{0}, t}\left(\vec{Y}_{\tau}\right)$ in terms of $\vec{X}_{t}$ and $\vec{Y}_{t}$. To this end, we differentiate (3.2) with respect to $t$ :

$$
\begin{gathered}
\hat{C}_{t_{0}, t}\left(\vec{Y}_{\tau}\right) \circ \overrightarrow{\exp } \int_{t_{0}}^{t} \vec{X}_{\tau} d \tau \circ\left(\vec{X}_{t}+\vec{Y}_{t}\right) \\
=\left(\frac{d}{d t} \hat{C}_{t_{0}, t}\left(\vec{Y}_{\tau}\right)\right) \circ \overrightarrow{\exp } \int_{t_{0}}^{t} \vec{X}_{\tau} d \tau+\hat{C}_{i_{0}, t}\left(\vec{Y}_{\tau}\right) \circ \overrightarrow{\exp } \int_{t_{0}}^{t} \vec{X}_{\tau} d \tau \circ \vec{X}_{t},
\end{gathered}
$$

whence

$$
\frac{d}{d t} \hat{C}_{t_{0}, t}\left(\vec{Y}_{\tau}\right)=\hat{C}_{t_{0}, t}\left(\vec{Y}_{\tau}\right) \circ\left(\operatorname{Ad} \overrightarrow{\exp } \int_{t_{0}}^{t} \vec{X}_{\tau} d \tau \vec{Y}_{t}\right)
$$

This differential equation was obtained by "variation of the constant $\hat{C}_{t_{0}, t}$ "; integrating it gives a representation of the left perturbation flow

$$
\hat{C}_{t_{0}, t}\left(\vec{Y}_{\tau}\right)=\overrightarrow{\exp } \int_{t_{0}}^{t}\left(\operatorname{Ad} \hat{P}_{t_{0}, \tau}\right) \vec{Y}_{\tau} d \tau=\overrightarrow{\exp } \int_{t_{0}}^{t}\left(\overrightarrow{\exp } \int_{t_{0}}^{\tau} \operatorname{ad} \overrightarrow{X_{\theta}} d \theta\right) \overrightarrow{Y_{\tau}} d \tau
$$


and, at the same time, a representation of the perturbed flow

$$
\overrightarrow{\exp } \int_{t_{0}}^{t}\left(\vec{X}_{\tau}+\vec{Y}_{\tau}\right) d \tau=\overrightarrow{\exp } \int_{t_{0}}^{t}\left(\operatorname{Ad} \hat{P}_{t_{0}, \tau}\right) \vec{Y}_{\tau} d \tau \circ \overrightarrow{\exp } \int_{t_{0}}^{t} \vec{X}_{\tau} d \tau
$$

To determine $\hat{D}_{t_{0, t}}\left(\vec{Y}_{\tau}\right)$, we write the following sequence of equalities (see (3.10), (3.6), (3.8) and (3.9)):

$$
\begin{gathered}
\overrightarrow{\exp } \int_{t_{0}}^{t}\left(\operatorname{Ad} \hat{P}_{t_{0}, \tau}\right) \vec{Y}_{\tau} d \tau \circ \overrightarrow{\exp } \int_{t_{0}}^{t} \vec{X}_{\tau} d \tau \\
=\overrightarrow{\exp } \int_{t_{0}}^{t} \vec{X}_{\tau} d \tau \circ \operatorname{Ad} \hat{P}_{t_{0}, t}^{-1} \overrightarrow{\exp } \int_{t_{0}}^{t}\left(\operatorname{Ad} \hat{P}_{t_{0}, \tau}\right) \vec{Y}_{\tau} d \tau \\
=\overrightarrow{\exp } \int_{t_{0}}^{t} \vec{X}_{\tau} d \tau \circ \overrightarrow{\exp } \int_{t_{0}}^{t}\left(\operatorname{Ad} \hat{P}_{t, t_{0}} \circ \operatorname{Ad} \hat{P}_{t_{0}, \tau}\right) \vec{Y}_{\tau} d \tau \\
=\overrightarrow{\exp } \int_{t_{0}}^{t} \vec{X}_{\tau} d \tau \circ \overrightarrow{\exp } \int_{t_{0}}^{t}\left(\operatorname{Ad} \hat{P}_{t, \tau}\right) \vec{Y}_{\tau} d \tau,
\end{gathered}
$$

from which we get

$$
\hat{D}_{t_{0}, t}\left(\vec{Y}_{\tau}\right)=\overrightarrow{\exp } \int_{t_{0}}^{t}\left(\operatorname{Ad} \hat{P}_{t, \tau}\right) \vec{Y}_{\tau} d \tau=\overrightarrow{\exp } \int_{t_{0}}^{t}\left(\overrightarrow{\exp } \int_{t}^{\tau} \operatorname{ad} \overrightarrow{X_{\theta}} d \theta\right) \vec{Y}_{\tau} d \tau
$$

Thus the perturbed flow can be represented with the help of the equalities

$$
\begin{aligned}
& \overrightarrow{\exp } \int_{t_{0}}^{t}\left(\vec{X}_{\tau}+\dddot{Y}_{\tau}\right) d \tau=\overrightarrow{\exp } \int_{t_{0}}^{t}\left(\operatorname{Ad} \overrightarrow{\exp } \int_{t_{0}}^{\tau} \vec{X}_{\theta} d \theta\right) \vec{Y}_{\tau} d \tau \circ \overrightarrow{\exp } \int_{t_{0}}^{t} \vec{X}_{\tau} d \tau \\
& =\overrightarrow{\exp } \int_{t_{0}}^{t}\left(\overrightarrow{\exp } \int_{t_{0}}^{\tau} \operatorname{ad} \vec{X}_{\theta} d \theta\right) \vec{Y}_{\tau} d \tau \circ \overrightarrow{\exp } \int_{t_{0}}^{t} \vec{X}_{\tau} d \tau \text {, } \\
& \overrightarrow{\exp } \int_{t_{0}}^{t}\left(\vec{X}_{\tau}+\vec{Y}_{\tau}\right) d \tau=\overrightarrow{\exp } \int_{t_{0}}^{t} \vec{X}_{\tau} d \tau \circ \overrightarrow{\exp } \int_{t_{0}}^{t}\left(\operatorname{Ad} \overrightarrow{\exp } \int_{t}^{\tau} \vec{X}_{\theta} d \theta\right) \vec{Y}_{\tau} d \tau \\
& =\overrightarrow{\exp } \int_{t_{0}}^{t} \vec{X}_{\tau} d \tau \circ \overrightarrow{\exp } \int_{t_{0}}^{t}\left(\overrightarrow{\exp } \int_{t}^{\tau} \operatorname{ad} \vec{X}_{\theta} d \theta\right) \vec{Y}_{\tau} d \tau \text {. }
\end{aligned}
$$

We call them generalized "variation of constants" formulas.

2. Asymptotic forms of (3.15) and (3.16). In these formulas, the expressions $\overrightarrow{\exp } \int_{t_{0}}^{\tau}$ ad $\vec{X}_{\theta} d \theta$ and $\overrightarrow{\exp } \int_{t}^{\tau}$ ad $\vec{X}_{\theta} d \theta$ do more than serve as formal definitions of the operators Ad $\hat{P}_{t_{0, \tau}}$ and Ad $\hat{P}_{t, \tau}$. In the form in which they are written, (3.15) and (3.16) point to the following two basic facts.

1) If $\vec{X}_{t}$ and $\vec{Y}_{t}$ are bounded analytic fields, then if we calculate all of the chronological exponentials on the right-hand sides of (3.15) and (3.16), as well as the corresponding formal Volterra series, and if we carry out the indicated operations, we arrive at formal series which when applied to the identity mapping $E$ give convergent series which determine the perturbed flow.

2) In the case of arbitrary fields $\vec{X}_{t}$ and $\vec{Y}_{t}$, this procedure does not lead to convergent series, but these series give an asymptotic representation of $\overrightarrow{\exp } \int_{t_{0}}^{t}\left(\vec{X}_{\tau}+\vec{Y}_{\tau}\right) d \tau$. We give the precise meanings of these statements in Propositions 3.1 and 3.2. 
Proposition 3.1. Assume that $\vec{X}_{t}, t \in \mathbf{R}$, is a uniformly integrable, bounded, analytic field, and that $\vec{Z}$ is a bounded analytic field: $X_{t} \in \Omega_{\sigma}^{n}$ for all $t \in \mathbf{R}$ and $Z \in \Omega_{\sigma}^{n}$. Then for all $\sigma^{\prime}<\sigma$ there exists a $\rho>0$ such that the series

$$
\begin{gathered}
\left(\overrightarrow{\mathcal{V}}_{t_{0}, t}\left(\operatorname{ad} \vec{X}_{\tau}\right) \vec{Z}\right) E(z)=Z(z) \\
+\sum_{m=1}^{\infty} \int_{t_{0}}^{t} d \tau_{1} \int_{t_{0}}^{\tau_{1}} d \tau_{2} \cdots \int_{t_{0}}^{\tau_{m-1}} d \tau_{m}\left(\operatorname{ad} \vec{X}_{\tau_{m}} \circ \cdots \circ \operatorname{ad} \vec{X}_{\tau_{1}} \vec{Z}\right) E(z)
\end{gathered}
$$

converges absolutely and uniformly in $t_{0}, t$, and $z$ for $\left|t-t_{0}\right| \leq \rho$ and $z \in V_{\sigma^{\prime}}$, and for these values of $t_{0}, t$, and $z$,

$$
\left(\operatorname{Ad} \overrightarrow{\exp } \int_{t_{0}}^{t} \vec{X}_{\tau} d \tau \vec{Z}\right) E(z)=\left(\vec{\mho}_{t_{0}, t}\left(\operatorname{ad} \vec{X}_{\tau}\right) \vec{Z}\right) E(z)
$$

The proof can be obtained from the estimate

$$
\begin{aligned}
& \| \int_{t_{0}}^{t} d \tau_{1} \int_{t_{0}}^{\tau_{1}} d \tau_{2} \ldots \int_{t_{0}}^{\tau_{m-1}} d \tau_{m}\left(\operatorname{ad} \vec{X}_{\tau_{m}} \circ \cdots \circ \text { ad } \vec{X}_{\tau_{1}} \vec{Z}\right) E \|_{\sigma^{\prime}}^{C^{n}} \\
& \leqslant 2^{m}\left(\frac{2 n}{\sigma-\sigma^{\prime}}\right)^{m}\left|\int_{t_{0}}^{t}\left\|X_{\tau}\right\|_{\sigma}^{c^{n}} d \dot{\tau}\right|^{m}\|Z\|_{\sigma}^{\mathbf{C}^{n}}
\end{aligned}
$$

which follows from the similar estimate (2.3).

Proposition 3.2. If $\vec{X}_{t}, t \in \mathbf{R}$, is locally integrable, then for $\vec{Z} \in \operatorname{Der}(\Phi)$

$$
\begin{gathered}
\|\left(\operatorname{Adexp} \int_{t_{0}}^{t} \vec{X}_{\tau} d \tau \vec{Z}\right) E \\
-\left(Z+\sum_{\alpha=1}^{m-1} \int_{t_{0}}^{t} d \tau_{1} \int_{t_{0}}^{\tau_{1}} d \tau_{2} \cdots \int_{t_{0}}^{\tau_{\alpha-1}} d \tau_{\alpha}\left(\operatorname{ad} \vec{X}_{\tau_{\alpha}} \circ \cdots \text { ad } \vec{X}_{\tau_{1}} \vec{Z}\right)\right) E \|_{s, K} \\
=3 n(2 s+2)^{s+1}(6 n)^{m}(2 s+2 m)^{m(s+m)} C_{1}^{2} e^{2 C_{2}}\left|\int_{t_{0}}^{t}\left\|X_{\tau}\right\|_{s+1} d \tau\right|_{C_{3}} \\
\times\|Z\|_{s+m, M} \frac{1}{m !}\left|\int_{t_{0}}^{t}\left\|X_{\tau}\right\|_{s+m, M} d \tau\right|^{m},
\end{gathered}
$$

where

$$
\begin{gathered}
C_{1}=(s+2)(2 s+2)^{s+1} n^{s+1}\left(1+n+\operatorname{diam} K+4\left|\int_{t_{0}}^{t}\left\|X_{\tau}\right\|_{0} d \tau\right|\right)^{s+1}, \\
C_{\mathbf{2}}=3 n(2 s+4)^{s+2} C_{1}, \quad C_{3}=d_{K}+\operatorname{diam} K+2\left|\int_{t_{0}}^{t}\left\|X_{\tau}\right\|_{0} d \tau\right|,
\end{gathered}
$$

where $d_{K}$ is the distance of the compact set $K \subset \mathbf{R}^{n}$ from the origin, and where $M=O_{R}(K)$ is a neighborhood of radius $R=2\left|\int_{t_{0}}^{t}\left\|X_{\tau}\right\|_{0} d \tau\right|$ of the compact set $K$. 
The proof is easy to construct if we take into account the identity

$$
\begin{aligned}
\operatorname{Ad} \hat{P}_{t_{0}, t} \vec{Z}-\left(\vec{Z}+\sum_{\alpha=1}^{m-1} \int_{t_{0}}^{t} d \tau_{1} \int_{t_{0}}^{\tau_{1}} d \tau_{2} \ldots \int_{t_{0}}^{\tau_{\alpha-1}} d \tau_{\alpha} \text { ad } \vec{X}_{\tau_{\alpha}} \circ \ldots \circ \text { ad } \vec{X}_{\tau_{1}} \vec{Z}\right) \\
=\int_{t_{0}}^{t} d \tau_{1} \int_{t_{0}}^{\tau_{1}} d \tau_{2} \cdots \int_{t_{0}}^{\tau_{m-1}} d \tau_{m} \operatorname{Ad} \hat{P}_{t_{0}, \tau_{m}} \circ \operatorname{ad} \vec{X}_{\tau_{m}} \circ \ldots \circ \operatorname{ad} \vec{X}_{\tau_{1}} \vec{Z}
\end{aligned}
$$

and make use of the following estimates, which follow directly from the corresponding estimates (1.9) and (2.9):

$$
\begin{gathered}
\|(\operatorname{ad} \vec{X} \vec{Z}) E\|_{s, K} \leqslant 6 n(2 s+2)^{s+1}\|X\|_{s+1, K}\left\|^{\prime}\right\|_{s+1, K}, \\
\left\|\left(\operatorname{ad} \vec{X}_{1} \circ \ldots \circ \operatorname{ad} \vec{X}_{m} \vec{Z}\right) E\right\|_{s, K} \leqslant \\
\leqslant(6 n)^{m}(2 s+2 m)^{m(s+m)}\left\|X_{1}\right\|_{s+m, K} \cdots\left\|X_{m}\right\|_{s+m, K}\|Z\|_{s+m, K}, \\
\left\|\left(\operatorname{Ad} \overrightarrow{\exp } \int_{t_{0}}^{t} \vec{X}_{\tau} d \tau \vec{Z}\right) E\right\|_{s, K} \leqslant 3 n(2 s+2)^{s+1} C_{1}^{2} e{ }^{2 C_{2}}\left|\int_{t_{0}}^{t}\left\|x_{\tau}\right\|_{s+1}^{d \tau}\right|_{C_{3}\|Z\|_{s, M} .}
\end{gathered}
$$

3. Calculation of the chronological logarithm. In this and the following subsection, we give two simple examples of the results we have obtained.

Since the field $\vec{X}_{t}, t \in \mathbf{R}$, is given uniquely in terms of the chronological exponential $\overrightarrow{\exp } \int_{t_{0}}^{t} \vec{X}_{\tau} d \tau$ which it generates by the formula

$$
\vec{X}_{t}=\left(\overrightarrow{\exp } \int_{t_{0}}^{t} \vec{X}_{\tau} d \tau\right)^{-1} \circ \frac{d}{d t} \overrightarrow{\exp } \int_{t_{0}}^{t} \vec{X}_{\tau} d \tau
$$

it is natural to call the function determined for flows $\overrightarrow{\exp } \int_{t_{0}}^{t} \vec{X}_{\tau} d \tau$ by the correspondence

$$
\overrightarrow{\exp } \int_{t_{0}}^{t} \vec{X}_{\tau} d \tau \mapsto \vec{X}_{t}
$$

a right chronological logarithm and to denote it by $\overrightarrow{\log } t_{0}$. Here $t_{0}$ plays the role of a parameter, so that the argument of a chronological logarithm is a function $t \mapsto$ $\overrightarrow{\exp } \int_{t_{0}}^{t} \vec{X}_{\tau} d \tau$ and its values are functions $t \mapsto \vec{X}_{t}$. Therefore to be perfectly precise we should write

$$
\overrightarrow{\log }_{t_{0}}\left\{\overrightarrow{\exp } \int_{t_{0}}^{t} \vec{X}_{\tau} d \tau ; t \in \mathbf{R}\right\}=\left\{\vec{X}_{\theta} ; \theta \in \mathbf{R}\right\},
$$

but we will use the less awkward notation

$$
\overrightarrow{\log } t_{0} \overrightarrow{\exp } \int_{t_{0}}^{t} \vec{X}_{\tau} d \tau=\vec{X}_{t}
$$

We define the left chronological logarithm similarly:

$$
\overleftarrow{\log } \bar{t}_{0} \leftarrow-\exp \int_{t_{0}}^{t} \vec{X}_{\tau} d \tau=\vec{X}_{t}
$$


Frequently it is necessary to calculate the field which generates a flow, i.e., the chronological logarithm of a flow when the flow is given not in "canonical" notation but in the form

$$
\hat{P}_{t_{0}, t}=\overrightarrow{\exp } \int_{t_{0}}^{t} \vec{Y}_{\tau}^{(1)} d \tau \circ \ldots \circ \overrightarrow{\exp } \int_{t_{0}}^{t} \bar{Y}_{\tau}^{(m)} d \tau .
$$

To do this, it suffices to write

$$
\begin{aligned}
& \overrightarrow{\log }_{t_{0}} \hat{P}_{t_{0}, t}=\hat{P}_{t_{0}, t}^{-1} \circ \frac{d}{d t} P_{t_{0}, t}
\end{aligned}
$$

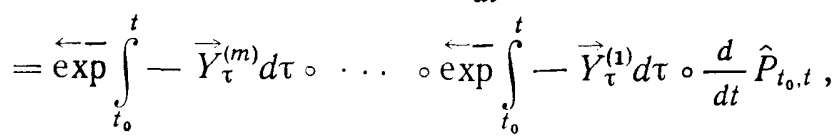

to represent the derivative $d \hat{P}_{t_{0}, t} / d t$ in the form of an appropriate sum of $m$ terms, and then to use (3.7). As a result, we get

$$
\begin{aligned}
& \overrightarrow{\log }_{t_{0}}\left(\overrightarrow{\exp } \int_{t_{0}}^{t} \vec{Y}_{\tau}^{(1)} d \tau \circ \ldots \overrightarrow{\exp } \int_{t_{0}}^{t} \vec{Y}_{\tau}^{(m)} d \tau\right) \\
& =\left(\overleftarrow{\exp } \int_{t_{0}}^{t}-\operatorname{ad} \vec{Y}_{\tau}^{(m)} d \tau \circ \ldots \circ \overleftarrow{\exp } \int_{t_{0}}^{t}-\operatorname{ad} \vec{Y}_{\tau}^{(2)} d \tau\right) \vec{Y}_{t}^{(1)}
\end{aligned}
$$

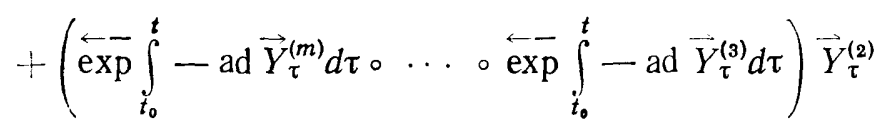

$$
\begin{aligned}
& +\cdots+\left(\overleftarrow{\exp } \int_{t_{0}}^{t}-\operatorname{ad} \vec{Y}_{\tau}^{(m)} d \tau\right) \vec{Y}_{t}^{(m-1)}+\vec{Y}_{\tau}^{(m)} .
\end{aligned}
$$

We find a right chronological logarithm as a solution of

$$
\frac{d}{d t} \hat{P}_{t_{0, t}}=\hat{P}_{t_{0}, t} \circ \vec{X}_{t}+\vec{Y}_{t} \circ \hat{P}_{t_{0}, t}
$$

We have

$$
\hat{P}_{t_{0}, t}=\overleftarrow{\exp } \int_{t_{0}}^{t} \vec{Y}_{\tau} d \tau \circ \overrightarrow{\exp } \int_{t_{0}}^{t} \vec{X}_{\tau} d \tau
$$

therefore, if we denote the unknown logarithm by $\vec{Z}_{t}$ and repeat the computations in the derivation of (3.17), we get

$$
\begin{gathered}
\vec{Z}_{t}=\left(-\overline{\exp } \int_{t_{0}}^{t} \vec{Y}_{\tau} d \tau \circ \overrightarrow{\exp } \int_{t_{0}}^{t} \vec{X}_{\tau} d \tau\right)^{-1} \circ \frac{d}{d t}\left(\overleftarrow{\exp } \int_{t_{0}}^{t} \vec{Y}_{\tau} d \tau \circ \overrightarrow{\exp } \int_{t_{0}}^{t} \vec{X}_{\tau} d \tau\right) \\
=\vec{X}_{t}+\left(\overleftarrow{\exp } \int_{t_{0}}^{t}-\operatorname{ad} \vec{X}_{\tau} d \tau \circ \overrightarrow{\exp } \int_{t_{0}}^{t}-\operatorname{ad} \vec{Y}_{\tau} d \tau\right) \vec{Y}_{t} .
\end{gathered}
$$

4. The quasistationary field case. We assume that the field $\vec{X}_{t}$ is stationary: $\vec{X}_{t}=\vec{X}$ for all $t$ in $\mathbf{R}$. We define the exponential of $\vec{X}$ by

$$
e^{\vec{X}}=\overrightarrow{\exp } \int_{0}^{1} \vec{X} d \tau
$$


The field $\vec{X}_{t}, t \in \mathbf{R}$, is called quasistationary if for some fixed $t_{0}$ it commutes with the field $\int_{t_{0}}^{t} \vec{X}_{\tau} d \tau$ for all $t$ in $\mathbf{R}$.

Proposition 3.3. If the field $\vec{X}_{t}, t \in \mathbf{R}$, is quasistationary,

$$
\left[\vec{X}_{t}, \int_{t_{0}}^{t} \vec{X}_{\tau} d \tau\right]=0 \quad \forall t \in \mathbf{R}
$$

then the family of diffeomorphisms

$$
\int^{t} \vec{t}^{t} \vec{x}_{\tau} d \tau, \quad t \in \mathbf{R}
$$

is a flow which satisfies

$$
\frac{d}{d t} e^{\int_{t_{0}}^{t} \vec{X}_{\tau} d \tau}=e^{\int_{t_{0}}^{t} \vec{X}_{\tau} d \tau} \circ \vec{X}_{t}=\vec{X}_{t} \circ e^{\int_{t_{0}}^{t} \vec{x}_{\tau} d \tau}
$$

consequently in this case

$$
\begin{aligned}
& \overrightarrow{\exp } \int_{t_{0}}^{t} \vec{X}_{\tau} d \tau=\ddot{\exp } \int_{t_{0}}^{t} \vec{X}_{\tau} d \tau=e^{\int_{t_{0}}^{t} \vec{X}_{\tau} d \tau} \\
& \left(\begin{array}{c}
\int_{t^{t}}^{t} \vec{X}_{\tau} d \tau \\
e^{t_{0}}
\end{array}\right)^{-1}=e^{-\int_{t_{0}}^{t} \vec{X}_{\tau} d \tau} \quad \forall t \in \mathbf{R}
\end{aligned}
$$

In particular,

$$
\begin{gathered}
\frac{d}{d t} e^{\left(t-t_{0}\right) \vec{X}}=e^{\left(t-t_{0}\right) \vec{X}} \circ \vec{X}=\vec{X} \circ e^{\left(t-t_{0}\right) \vec{X}} \\
\left(e^{\left(t-t_{0}\right) \vec{X}}\right)^{-1}=e^{\left(t_{0}-t\right) \vec{X}}
\end{gathered}
$$

Proof. We calculate the expression

$$
\frac{1}{\delta t}\left(\int_{e^{t_{0}}}^{t} \vec{x}_{\tau} d \tau+\int_{t}^{t+\delta t} \vec{x}_{\tau} d \tau-\int_{t^{t_{0}}}^{t} \vec{x}_{\tau} d \tau\right) .
$$

\section{Putting}

$$
\vec{Y}=\int_{t_{0}}^{t} \vec{X}_{\tau} d \tau, \vec{Y}_{\delta t}=\int_{t}^{t+\delta t} \vec{X}_{\tau} d \tau
$$

for brevity, and making use of (3.16), we get

$$
\begin{aligned}
& \frac{1}{\delta t}\left(e^{\vec{Y}+\vec{Y}_{\delta t}}-e^{\vec{Y}}\right)=\frac{1}{\delta t}\left(\overrightarrow{\exp } \int_{0}^{1}\left(\vec{Y}+\vec{Y}_{\delta t}\right) d \tau-\overrightarrow{\exp } \int_{0}^{1} \vec{Y} d \tau\right) \\
& \quad=\frac{1}{\delta t} \overrightarrow{\exp } \int_{0}^{1} \vec{Y} d \tau \circ\left(\overrightarrow{\exp } \int_{0}^{1}\left(\operatorname{Ad} \overrightarrow{\exp } \int_{1}^{\tau} \vec{Y} d \theta\right) \vec{Y}_{\delta t} d \tau-\mathrm{Id}\right) .
\end{aligned}
$$


For $\delta t \rightarrow 0,(2.23)$ (Proposition 2.3) gives, at each point of differentiability of $\int_{t_{0}}^{t} \vec{X}_{\tau} d \tau$, that

$$
\frac{1}{\partial t}\left(\overrightarrow{\exp } \int_{0}^{1}\left(\operatorname{Ad} \overrightarrow{\exp } \int_{1}^{\tau} \vec{Y} d \theta\right) \vec{Y}_{\delta t} d \tau-\mathrm{Id}\right) \rightarrow \int_{0}^{1}\left(\operatorname{Ad} \overrightarrow{\exp } \int_{1}^{\tau} \vec{Y} d \theta\right) \vec{X}_{t}
$$

consequently as $\delta t \rightarrow 0,(3.20)$ approaches (see (3.11))

$$
\overrightarrow{\exp } \int_{0}^{1} \vec{Y} d \tau \circ \int_{0}^{1}\left(\operatorname{Ad} \overrightarrow{\exp } \int_{1}^{\tau} \vec{Y} d \theta\right) \vec{X}_{t}=\overrightarrow{\exp } \int_{0}^{1} \vec{Y} d \tau \circ \vec{X}_{t}
$$

which proves the first equality of (3.19). If in this proof we use (3.15) in place of (3.16), we get the second equality of (3.19).

For quasistationary fields, (3.5), (3.15) and (3.16) have the form

$$
\begin{aligned}
& \operatorname{Ad} e^{\int_{t_{0}}^{t} \vec{x}_{\tau} d \tau} \vec{Z}=e^{\int_{0}^{t} \operatorname{ad} \vec{X}_{\tau} d \tau} \vec{Z}
\end{aligned}
$$

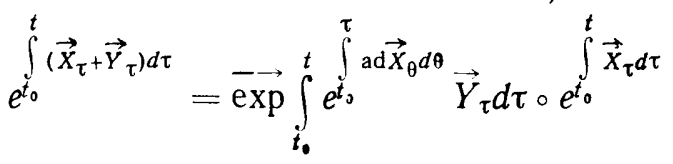

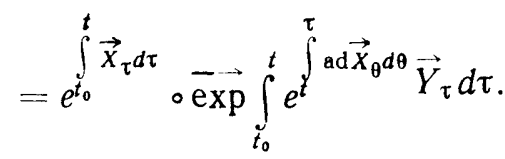

In particular, for stationary fields we get the identities

$$
\begin{gathered}
e^{t \vec{X}} \circ \vec{Z} \circ e^{-t \vec{X}}=e^{t \mathrm{ad} X} \vec{Z}, \\
e^{t(\vec{X}+\vec{Y})}=\overrightarrow{\exp } \int_{0}^{t} e^{\tau \mathrm{ad} \vec{X}} \vec{Y} d \tau \circ e^{t \vec{X}} \\
=e^{t \vec{X}} \cdot \overrightarrow{\exp } \int_{0}^{t} e^{(\tau-t) \mathrm{ad} \vec{X}} \vec{Y} d \tau,
\end{gathered}
$$

which are frequently useful.

\section{\$4. Variation of flows}

We will assume that the flow $\overrightarrow{\exp } \int_{t_{0}}^{t} \vec{Y}_{\tau} d \tau$, which we will consider here as a perturbation of the identity flow.Id ${ }_{t} \equiv$ Id (generated by the zero field), is given, but we will regard $\vec{Y}_{t}$ as a perturbation of the zero field. We will consider $\overrightarrow{\exp } \int_{t_{0}}^{t} \vec{Y}_{\tau} d \tau$ as the corresponding (right or left) perturbation flow. We pose the following problem.

For a given flow $\overrightarrow{\exp } \int_{t_{0}}^{t} Y_{\tau} d \tau$, find a field $V_{t_{0}, t}\left(\vec{Y}_{\tau}\right)$ which, in some asymptotic sense to be specified precisely in Proposition 4.1, satisfies

$$
\overrightarrow{\exp } \int_{t_{0}}^{t} \vec{Y}_{\tau} d \tau \cong e^{v_{t_{0}, t}\left(\vec{Y}_{\tau}\right)}
$$

where the function of a stationary field in the exponent is to be understood in the sense defined by (3.18). 
If such a field $V_{t_{0}, t}\left(\vec{Y}_{\tau}\right)$ exists, it is natural to call it the logarithm (not chronological!) of the flow $\overrightarrow{\exp } \int_{t_{0}}^{t} \vec{Y}_{\tau} d \tau$ and to denote it by

$$
V_{t_{0} . t}\left(\vec{Y}_{\tau}\right)=\ln \overrightarrow{\exp } \int_{t_{0}}^{t} \vec{Y}_{\tau} d \tau
$$

It turns out that a reasonable solution of the problem stated above requires the extension of the concept of a nonstationary field by the introduction of formal vector fields by means of chronological series.

An explicit algorithm to be described in $\$ 5.2$ gives a "universal" sequence of polynomials in noncommutative variables

$$
\mathfrak{g}_{1}\left(\zeta_{1}\right), \mathfrak{g}_{2}\left(\zeta_{1}, \zeta_{2}\right), \ldots, \mathfrak{g}_{m}\left(\zeta_{1}, \ldots, \zeta_{m}\right), \ldots,
$$

where $\mathfrak{g}_{m}$ is homogeneous of degree 1 in each variable. In addition, all of the polynomials $\mathfrak{B}_{m}$ have a remarkable property: they are "commutator" polynomials in their variables. A commutator polynomial is one which can be expressed as a linear combination of the variables $\zeta_{1}, \ldots, \zeta_{m}$, of their commutators $\left[\zeta_{j}, \zeta_{k}\right]=\zeta_{j} \zeta_{k}-\zeta_{k} \zeta_{j}$, of the commutators of these, and so forth. For example, the first three polynomials have the form

$$
\begin{gathered}
\mathfrak{g}_{1}\left(\zeta_{1}\right)=\zeta_{1}, \quad \mathfrak{g}_{2}\left(\zeta_{1}, \zeta_{2}\right) \mp \frac{1}{2}\left[\zeta_{2}, \zeta_{1}\right], \\
\left.\mathfrak{g}_{3}\left(\zeta_{1}, \zeta_{2}, \zeta_{3}\right)=\frac{1}{6}\left(\left[\zeta_{3}, \mid \zeta_{2}, \zeta_{1}\right]\right]+\left[\left[\zeta_{3}, \zeta_{2}\right], \zeta_{1}\right]\right)
\end{gathered}
$$

It is clear that if $\vec{Y}_{1}, \ldots, \vec{Y}_{m}$ are arbitrary fields, then the expression $g_{m}\left(\vec{Y}_{1}, \ldots, \vec{Y}_{m}\right)$ will be a field.

With the help of $\vec{Y}_{t}$ we construct the formal chronological series

$$
\begin{gathered}
V_{t_{0}, t}\left(\vec{Y}_{\tau}\right)=\sum_{m=1}^{\infty} V_{t_{0}, t}^{(m)}\left(\vec{Y}_{\tau}\right), \\
V_{t_{0}, t}^{(m)}\left(\vec{Y}_{\tau}\right)=\int_{t_{0}}^{t} d \tau_{1} \int_{t_{0}}^{\tau_{1}} d \tau_{2} \cdots \int_{t_{0}}^{\tau_{m-1}} d \tau_{m} g_{m}\left(\vec{Y}_{\tau_{1}}, \ldots, \vec{Y}_{\tau_{m}}\right),
\end{gathered}
$$

and we call it the formal vector field corresponding to the field $\vec{Y}_{t}$. From what was said above, all expressons $V_{t_{0} t}^{(m)}\left(\vec{Y}_{\tau}\right), m=1,2, \ldots$, represent vector fields in the ordinary sense.

The following proposition gives the precise sense in which the asymptotic relation (4.1) must be understood; the proof is given in $\$ 5.1$.

Proposition 4.1. If

$$
\int_{t_{0}}^{t}\left\|Y_{\tau}\right\|_{s: m} d \tau \leqslant 1
$$

then

$$
\left\|\left(\overrightarrow{\exp } \int_{t_{0}}^{t} Y_{\tau} d \tau-e^{\sum_{\alpha=1}^{m} v_{t_{0}, t}^{(\alpha)}\left(\vec{Y}_{\tau}\right)}\right) \varphi\right\|_{s, K} \leqslant C_{1}\left|\int_{t_{0}}^{t}\left\|Y_{\tau}\right\|_{s+2 m} d \tau\right|^{m+1}\|\varphi\|_{s+m+1, o_{C_{2}}(K)},
$$


where the constants $C_{1}$ and $C_{2}$ depend only on $s, m$, and diam $K$, and where $O_{C_{2}}(K)$ is a $C_{2}$-neighborhood of the compact set $K$.

As an immediate consequence of (4.5) and (2.23), we can conclude that if a point $x \in \mathbf{R}^{n}$

$$
Y_{t_{0}, t}^{(\alpha)}\left(Y_{\tau}\right) E(x)=0 \quad \forall \alpha=1, \ldots, m-1
$$

then

$$
\overrightarrow{\exp } \int_{i_{0}}^{t} \vec{Y}_{\tau} d \tau E(x)=x+V_{t_{0}, t}^{(m)}\left(\vec{Y}_{\tau}\right) E(x)+O\left(\left(\int_{t_{0}}^{t}\left\|Y_{\tau}\right\|_{s+2 m} d \tau\right)^{m+1}\right)
$$

since if $\vec{Y}_{1}, \ldots, \vec{Y}_{k}$ are arbitrary fields and if $\vec{Y}_{1}$ vanishes at $x\left(\vec{Y}_{1}(x)=0\right)$, then the composition $\vec{Y}_{1} \circ \cdots \circ \vec{Y}_{k}$ vanishes at $x$. Thus if all of the functions $V_{t_{0}, t}^{(\alpha)}\left(\vec{Y}_{\tau}\right) E, \alpha=$ $1, \ldots, m-1$, vanish at $x$, then the value of the perturbation field $\overrightarrow{\exp } \int_{t_{0}}^{t} \vec{Y}_{\tau} d \tau E$ at $x$ can be calculated by means of the expression $x+V_{t_{0}, t}^{(m)}\left(\vec{Y}_{\tau}\right) E(x)$, and the error can be estimated by means of (4.7). This motivates the following terminology.

We call the field $V_{t_{0} I}^{(m)}\left(\vec{Y}_{\tau}\right)$ the mth variation of the identity flow corresponding to the perturbation field $\vec{Y}_{t}$, and we call the formal field $V_{t_{0}, t}\left(\vec{Y}_{\tau}\right)$ the complete variation of the identity flow. We inroduce the corresponding notation

$$
V_{t_{0}, t}^{(m)}\left(Y_{\tau}\right)=\delta^{(m)} \operatorname{Id}_{t_{0}, t}\left(\bar{Y}_{\tau}\right), V_{t_{0}, t}\left(Y_{\tau}\right)=\delta \operatorname{Id}_{t_{0}, t}\left(Y_{\tau}\right)
$$

and rewrite (4.1) and (4.4) in the form

$$
\overrightarrow{\exp } \int_{t_{0}}^{t} Y_{\tau} d \tau \simeq e^{\delta \mathrm{Id} t_{0}, t\left(\vec{Y}_{\tau}\right)}=\mathrm{Id}+\sum_{m=1}^{\infty} \frac{1}{m !}\left(\delta \operatorname{Id}_{t_{0}, t}\left(\vec{Y}_{\tau}\right)\right)^{m}
$$

$\delta \operatorname{Id}_{t_{0}, t}\left(\vec{Y}_{\tau}\right)=\sum_{m=1}^{\infty} \delta^{(m)} \operatorname{Id}_{t_{0}, t}\left(\vec{Y}_{\tau}\right)=\sum_{m=1}^{\infty} \int_{t_{0}}^{t} d \tau_{1} \int_{t_{0}}^{\tau_{1}} d \tau_{2} \ldots \int_{i_{0}}^{\tau_{m-1}} d \tau_{m} \mathfrak{g}_{m}\left(Y_{\tau_{1}}, \ldots, \vec{Y}_{\tau_{m}}\right)$.

We call this representation the Maclaurin expansion ("in the vicinity of the zero field") of the perturbation flow $\overrightarrow{\exp } \int_{t_{0}}^{t} \vec{Y}_{\tau} d \tau$.

Combining (4.8) and (3.17), we arrive at the following generalization of the CampbellHausdorff formula:

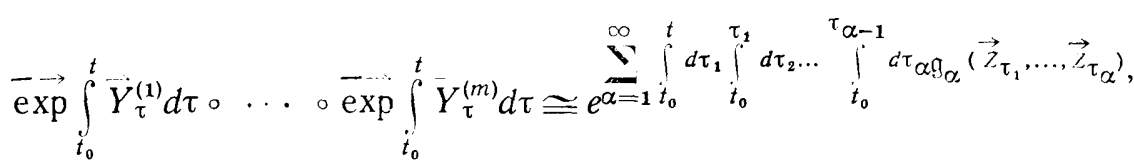

$$
\begin{aligned}
& \vec{Z}_{t}=\left(\overleftarrow{\exp } \int_{t_{0}}^{t}-\operatorname{ad} \vec{Y}_{\tau}^{(m)} d \tau \circ \ldots \circ \exp \int_{i_{0}}^{t}-\operatorname{ad} \vec{Y}_{\tau}^{(2)} d \tau\right) \vec{Y}_{t}^{(1)} \\
& +\cdots+\left(-\overline{\exp } \int_{t_{0}}^{t}-\operatorname{ad} \vec{Y}_{\tau}^{(m)} d \tau\right) \vec{Y}_{t}^{(m-1)}+\vec{Y}_{t}^{(m)} .
\end{aligned}
$$

The usual computational form of this formula, which, as a rule, is stated only for the case where $\vec{Y}_{t}^{(i)} \equiv \vec{Y}_{j}$ are stationary fields, $j=1,2$, is extremely complicated because the $\alpha$-fold integrations, only indicated in (4.9), are actually carried out. 
The question of convergence of the formal series on the right-hand sides of the asymptotic relations (4.8) and (4.9) and the question of precise equality to the flows on the left-hand sides are treated in the next section.

The expression

$$
e^{V_{t_{0}, t}\left(\vec{Y}_{\tau}\right)}=\mathrm{Id}+\sum_{m=1}^{\infty} \frac{1}{m !}\left(V_{t_{0}, t}\left(\vec{Y}_{\tau}\right)\right)^{m},
$$

where $V_{t_{0} t}\left(\vec{Y}_{\tau}\right)$ is an arbitrary formal field, is called the formal flow corresponding to the formal field $V_{t_{0}, t}\left(\vec{Y}_{\tau}\right)$. We can introduce a multiplication operation into the set of all formal flows by means of the relation

$$
e^{V_{t_{0}, t}\left(\vec{Y}_{\tau}^{\prime}\right)} e^{V_{t_{0}, t}\left(\vec{Y}_{\tau}^{\prime \prime}\right)}=e^{V_{t_{0}, t}\left(\lambda_{1}\left(\vec{Y}_{\tau}^{\prime}, \vec{Y}_{\tau}^{\prime \prime}\right)\right)}
$$

where (cf. (3.17))

$$
\lambda\left(\vec{Y}_{t}^{\prime}, \vec{Y}_{t}^{\prime \prime}\right)=\left(\overleftarrow{\exp } \int_{t_{0}}^{t}-\operatorname{ad} \vec{Y}_{\tau}^{\prime \prime} d \tau\right) \vec{Y}_{t}^{\prime}+\vec{Y}_{t}^{\prime \prime}
$$

which then makes it into a multiplicative group, as is easy to see.

One decided advantage of the definitions of variations given here over the usual definitions is that our variations satisfy the asymptotic relations (4.5)-(4.7) and have an invariant form: the expressions $\delta^{(m)} \mathrm{Id}_{t_{0, t}}\left(\vec{Y}_{\tau}\right)$ represent vector fields, which therefore act as "infinitesimal shifts" not only in $\Phi$ but also in $\mathbf{R}^{\text {n }}$.

The "usual variations" of a perturbation flow, successive terms in the "Volterra expansion"

$$
\overrightarrow{\exp } \int_{t_{0}}^{t} \vec{Y}_{\tau} d \tau \cong \mathrm{Id}+\int_{t_{0}}^{t} d \tau_{1} \vec{Y}_{\tau_{1}}+\int_{t_{0}}^{t} d \tau_{1} \int_{t_{0}}^{\tau_{1}} d \tau_{2} \vec{Y}_{\tau_{2}} \circ \vec{Y}_{\tau_{1}}+\ldots,
$$

fail to have an invariant meaning beginning with the quadratic term; therefore they act only in $\Phi$, and not in $\mathbf{R}^{n}$. By means of the procedure described here (see also the construction of the polynomials $\mathfrak{g}_{m}$ in the next section), we actually "extract" the invariant variations $\delta^{(m)} \operatorname{Id}_{t_{0}, t}\left(\vec{Y}_{\tau}\right)$ from the "usual" ones

$$
\int_{i_{0}}^{t} d \tau_{1} \int_{t_{0}}^{\tau_{1}} d \tau_{2} \cdots \int_{t_{0}}^{\tau_{m-1}} d \tau_{m} \vec{Y}_{\tau_{m}} \circ \cdots \circ \bar{Y}_{\tau_{1}}
$$

Their interrelation is given explicitly by (4.8).

For example,

$$
\begin{gathered}
\delta^{(1)} \operatorname{Id}_{t_{0}, t}\left(\vec{Y}_{\tau}\right)=\int_{t_{0}}^{t} \vec{Y}_{\tau} d \tau, \\
\delta^{(2)} \operatorname{Id}_{t_{0}, t}\left(\vec{Y}_{\tau}\right)=\int_{t_{0}}^{t} d \tau_{1} \int_{t_{0}}^{\tau_{1}} d \tau_{2} \vec{Y}_{\tau_{2}} \circ \vec{Y}_{\tau_{1}}-\frac{1}{2} \delta^{(1)} \operatorname{Id}_{t_{0}, t}\left(\vec{Y}_{\tau}\right) \circ \delta^{(1)} \operatorname{Id}_{t_{0}, t}\left(\vec{Y}_{\tau}\right),
\end{gathered}
$$

and so forth.

In conclusion we note that if $\vec{Y}_{t}$ is a perturbation of an arbitrary flow $\hat{P}_{t_{0}, t}=$ $\overrightarrow{\exp } \int_{t_{0}}^{t} \vec{X}_{\tau} d \tau$ instead of the identity flow, then the corresponding variation of $\hat{P}_{t_{0, t}}$ must 
be defined by

$$
\begin{aligned}
\delta^{(m)} \hat{P}_{t_{0}, t}\left(\vec{Y}_{\tau}\right)= & \int_{t_{0}}^{t} d \tau_{1} \int_{t_{0}}^{\tau_{1}} d \tau_{2} \ldots \int_{t_{0}}^{\tau_{m-1}} d \tau_{m} g_{m}\left(\left(\overrightarrow{\exp } \int_{t_{0}}^{\tau_{1}} \operatorname{ad} \vec{X}_{\theta} d \theta\right) \vec{Y}_{\tau_{1}}, \ldots,\right. \\
& \left.\left(\overrightarrow{\exp } \int_{t_{0}}^{\tau_{m}} \operatorname{ad} X_{\theta} d \theta\right) \vec{Y}_{\tau_{m}}\right),
\end{aligned}
$$

and the complete variation by

$$
\delta \overrightarrow{\exp } \int_{t_{0}}^{t} \vec{X}_{\tau} d \tau\left(\vec{Y}_{\tau}\right)=\sum_{m=1}^{\infty} \delta^{(m)} \hat{P}_{t_{0}, t}\left(\vec{Y}_{\tau}\right)
$$

we get for the expansion of the perturbation flow in a "Taylor series in the vicinity of $X_{t}$ " the expression

$$
\overrightarrow{\exp } \int_{t_{0}}^{t}\left(\overrightarrow{\exp } \int_{t_{0}}^{\tau} \operatorname{ad} \vec{X}_{\theta} d \theta\right) \vec{Y}_{\tau} d \tau \cong e^{\delta \hat{P}_{t_{0}, t}\left(\vec{Y}_{\tau}\right)}
$$

As the "Taylor expansion" of the perturbed flow we get

$$
\overrightarrow{\exp } \int_{t_{0}}^{t}\left(\vec{X}_{\tau}+\vec{Y}_{\tau}\right) d \tau \cong e^{\delta \hat{P}_{t_{0}, t}\left(\vec{Y}_{\tau}\right)} \hat{P}_{t_{0}, t}
$$

\$5. Calculation of the formal field $\left({ }^{1}\right) V_{t_{0}, t}\left(\vec{X}_{\tau}\right)=\ln \overrightarrow{\exp } \int_{t_{0}}^{t} \vec{X}_{\tau} d \tau$

We assume throughout this section that all differentiations and integrations of formal series are to be performed termwise.

1. Computation in the algebra $\mathbb{Q}$ of chronological series. In the algebra $\mathbb{Q}$ of formal chronological series, we denote by $\Re$ the ideal consisting of series of the form

$$
\breve{E}_{t_{0}, t}\left(X_{\tau}\right)=\sum_{m=1}^{\infty} \int_{t_{0}}^{t} d \tau_{1} \int_{t_{0}}^{\tau_{1}} d \tau_{2} \ldots \int_{t_{0}}^{\tau_{m-1}} d \tau_{m} c_{m}\left(\vec{X}_{t}, \vec{X}_{\tau_{1}}, \ldots, \vec{X}_{\tau_{m}}\right),
$$

i.e., series without zero terms. The $k$ th power of $\mathfrak{T}$ is denoted by $\mathfrak{N}^{k}$. We call a series $\mathfrak{E}_{t_{0, t}}\left(\vec{X}_{\tau}\right)$ in $\mathfrak{T}$ absolutely continuous if the polynomials $c_{m}\left(\zeta_{0}, \ldots, \zeta_{m}\right)$ do not depend on $\zeta_{0}$. It is clear that absolutely continuous series can be differentiated with respect to $t$, and the operation $d / d t$ maps the collection of all absolutely continuous series onto all of $\mathbb{Q}$.

Furthermore, if $\mathbb{E}_{t_{0}, t}\left(\vec{X}_{\tau}\right) \in \mathfrak{R}$, then

$$
e^{\mathfrak{E}_{t_{0}, t}\left(\vec{X}_{\tau}\right)} \stackrel{\text { def }}{=} \sum_{m=0}^{\infty} \frac{1}{m !}\left(\mathcal{E}_{t_{0}, t}\left(\ddot{X}_{\tau}\right)\right)^{m}
$$

is obviously a well-defined formal chronological series.

Proposition 5.1. There exists an absolutely continuous formal chronological series

$$
V_{t_{0}, t}\left(\vec{X}_{\tau}\right)=\sum_{m=1}^{\infty} \int_{t_{0}}^{t} d \tau_{1} \int_{t_{0}}^{\tau_{1}} d \tau_{2} \ldots \int_{t_{0}}^{\tau_{m-1}} d \tau_{m} g_{m}\left(\vec{X}_{\tau_{1}}, \ldots, \vec{X}_{\tau_{m}}\right),
$$

(1) We note that an explicit expression for $\ln \overrightarrow{\exp } \int_{t_{0}}^{t} \vec{X}_{\tau} d \tau$ is obtained in [9], but it is not in the form of a chronological series. The connection between this formula and the Campbell-Hausdorff formula is also noted there. 
such that

$$
\overrightarrow{\mathfrak{V}}_{t_{0}, t}\left(\vec{X}_{\tau}\right)=e^{V_{t_{0}, t}\left(\vec{X}_{\tau}\right)}
$$

In addition, the polynomials $\mathrm{g}_{m}\left(\zeta_{1}, \ldots, \zeta_{m}\right)$ are commutators (see $\left.\$ 4\right)$ and are homogeneous of the first degree in each variable.

The series $V_{t_{0}, t}\left(\vec{X}_{\tau}\right)$ whose existence is asserted in this proposition satisfies all of the hypotheses of Proposition 4.1. Indeed, let

$$
V_{t_{0}, t}^{(m)}\left(X_{\tau}\right)=\int_{t_{0}}^{t} d \tau_{1} \int_{t_{0}}^{\tau_{1}} d \tau_{2} \ldots \int_{t_{0}}^{\tau_{m-1}} d \tau_{m} g_{m}\left(\vec{X}_{\tau_{1}}, \ldots, \vec{X}_{\tau_{m}}\right)
$$

Since the commutator polynomial $g_{m}$ is homogeneous of the first degree in each variable, it follows from the inequality

$$
\|[\vec{X}, \vec{Y}] E\|_{s, k} \leqslant 6 n(2 s+2)^{s+1}\|X\|_{s+1}\|Y\|_{s+1},
$$

which holds for any $\vec{X}$ and $\vec{Y}$, that

$$
\left\|g_{m}\left(\vec{X}_{\tau_{1}}, \ldots, \vec{X}_{\tau_{m}}\right) E\right\|_{s, K} \leqslant C(s, m)\left\|X_{\tau_{1}}\right\|_{s+m, K} \cdots\left\|X_{\tau_{m}}\right\|_{s+m, K}
$$

Therefore

$$
\left\|V_{t_{0}, t}^{(m)}\left(\ddot{X}_{\tau}\right) E\right\|_{s, K}=\frac{C(s, m)}{m !}\left(\int_{t_{0}}^{t}\left\|X_{\tau}\right\|_{s+m, K} d \tau\right)^{m} .
$$

But then (4.5) follows at once from the formal equality (5.1) and the asymptotic estimate (2.23).

Proof of Proposition 5.1. It is required to find an absolutely continuous chronological series satisfying (5.1). In what follows, we will, as a rule, omit the arguments $t_{0}$ and $\vec{X}_{\tau}$ in $V_{t_{0}, t}\left(\vec{X}_{\tau}\right)$.

We differentiate (5.1) with respect to $t$. On the left we get $\vec{\vartheta}_{t_{0}, t}\left(\vec{X}_{\tau}\right) \circ \vec{X}_{t}$. In order to differentiate the right-hand side, we must use the formal variant of the variation formula (3.21). We have

$$
e^{V_{t+\varepsilon}}=e^{V_{t}+\int_{t}^{t+\varepsilon} \frac{d}{d \tau} V_{\tau} d \tau}=e^{V_{t}} \vec{\vartheta}_{0,1}\left(e^{-\tau \operatorname{ad} V_{t}} \int_{t}^{t+\varepsilon} \frac{d}{d \theta} V_{\theta} d \theta\right)
$$

Consequently

$$
\frac{d}{d t} e^{V_{t}}=e^{V_{t}} \circ \int_{0}^{1} e^{-\tau \mathrm{ad} V_{t}} d \tau \frac{d}{d t} V_{t}
$$

Thus it follows from (5.1) that

$$
\overrightarrow{\mathfrak{V}}_{t_{0}, t}\left(\vec{X}_{\tau}\right) \circ \vec{X}_{t}=e^{V_{t}} \circ \int_{0}^{1} e^{-\tau \operatorname{ad} V_{t}} d \tau \frac{d}{d t} V_{t}
$$


Cancelling the invertible series $\vec{\vartheta}_{t_{0}, t}\left(\vec{X}_{\tau}\right)=e^{V_{t}}$ from both sides, we get

$$
\vec{X}_{t}=\int_{0}^{1} e^{-\tau \operatorname{ad} V_{t}} d \tau \frac{d}{d t} V_{t}
$$

The series $\int_{0}^{1} e^{-\tau \text { ad } V_{t}} d \tau$ begins with Id, so it is invertible. The inverse series is the Maclaurin series of the function

$$
1 / \int_{0}^{1} e^{-\tau z} d \tau=\frac{-z}{e^{-z}-1}
$$

with the variable $z$ replaced by ad $V_{t}$. We put $\varphi(z)=-z /\left(e^{-z}-1\right)$. Our equation takes the form

$$
\frac{d}{d t} V_{t}=\varphi\left(\operatorname{ad} V_{t}\right) \vec{X}_{t}
$$

Here

$$
\varphi\left(\operatorname{ad} V_{t}\right)=\sum_{\alpha=0}^{\infty} \frac{\varphi^{(\alpha)}(0)}{\alpha !} \operatorname{ad}^{\alpha} V_{t}=1+\frac{1}{2} \operatorname{ad} V_{t}+\sum_{\alpha=2}^{\infty} \frac{B_{\alpha}}{\alpha !} \operatorname{ad}^{\alpha} V_{t},
$$

where $B_{\alpha}$ is the $\alpha$ th Bernoulli number: $B_{2}=\frac{1}{6}, B_{4}=-\frac{1}{3}, \ldots$, and $B_{2 \alpha+1}=0$ for $\alpha=$ $1,2, \ldots$

Conversely, we assume that the chronological series $V_{t}$ of $\mathfrak{T}$ satisfies (5.2). Then

$$
\frac{d}{d t} e^{V_{t}}=e^{V_{t}} \circ \int_{0}^{1} e^{-\tau \operatorname{ad} V_{t}} d \tau \frac{d}{d t} V_{t}=e^{V_{t}} \circ \int_{0}^{1} e^{-\tau \operatorname{ad} V_{t}} d \tau \varphi\left(\operatorname{ad} V_{t}\right) \vec{X}_{t}=e^{V_{t}} \circ \vec{X}_{t},
$$

and consequently $e^{V_{t}}=\overrightarrow{\widetilde{V}}_{t_{0}, t}\left(\vec{X}_{\tau}\right)$. Thus (5.1) is equivalent to (5.2).

To solve (5.2) it is convenient to introduce a formal Laurent series in the $m$-dimensional variable $\lambda=\left(\lambda_{1}, \ldots, \lambda_{m}\right)$ with coefficients in $\mathscr{Q}$. We denote by $\mathbb{Q}_{m}(\lambda)$ the collection of all series of the form

$$
\mathfrak{L}(\lambda)=\sum_{\left\llcorner\in \mathbf{Z}^{(m)}\right.} Q_{t_{0}, t}^{(\iota)}\left(\vec{X}_{\tau}\right) \lambda^{\iota}, \quad Q_{t_{0}, t}^{(\llcorner)}\left(\vec{X}_{\tau}\right) \in \mathbb{Q} .
$$

Here $\iota=\left(\iota_{1}, \ldots, \iota_{m}\right)$ is a multi-index, $\lambda^{\iota}=\lambda_{1}^{\iota_{1}}, \ldots, \lambda_{m}^{\iota_{m}}$, and $\mathbf{Z}^{(m)}$ is the set of all $m$-dimensional vectors with integer coordinates.

It is not possible to multiply arbitrary formal Laurent series, as is done with power series. Therefore $Q_{m}(\lambda)$ does not have a natural ring structure. Nevertheless we can say the following.

Let $\stackrel{0}{\mathscr{Q}}_{m}(\lambda)$ be the subset of $\mathbb{Q}_{m}(\lambda)$ defined by the following condition: a series

$$
\Re(\lambda)=\sum_{\iota \in Z^{(m)}} R_{t_{0}, t}^{(\iota)}\left(\vec{X}_{\tau}\right) \lambda^{\iota}
$$

in $\mathscr{Q}_{m}(\lambda)$ belongs to $\stackrel{0}{\mathbb{Q}}_{m}(\lambda)$ if and only if the coefficients $R_{t_{0}, t}^{(t)}\left(\vec{X}_{\tau}\right) \in \mathfrak{N}^{k_{\triangleleft}}$, where $k_{\iota} \rightarrow \infty$ as $|\iota| \rightarrow \infty$.

For any

$$
\mathfrak{L}(\lambda)=\sum_{\iota \in \mathbf{Z}^{(m)}} Q_{t_{0}, t}^{(\iota)}\left(\vec{X}_{\iota}\right) \lambda^{\iota} \in \mathbb{Q}_{m}(\lambda) \text { and } \Re(\lambda)=\sum_{\iota \in Z^{(m)}} R_{t_{0}, t}^{(\iota)}\left(\ddot{X}_{\iota}\right) \lambda^{\iota} \in \stackrel{\mathscr{Q}}{m}_{m}(\lambda)
$$


we define the product $\mathfrak{R N}(\lambda) \in \mathbb{Q}(\lambda)$ by

$$
\mathfrak{L N}(\lambda)=\sum_{\iota_{\in} \mathbf{Z}^{(m)}}\left(\sum_{\iota^{\prime}+\iota^{\prime \prime}=\iota} Q_{t_{0}, t}^{\left(\iota^{\prime}\right)} R_{t_{0}, t}^{\left(\iota^{\prime \prime}\right)}\right) \lambda^{\iota} .
$$

The infinite sum

$$
\sum_{\iota^{\prime}+\iota^{\prime \prime}=\iota} Q_{t_{0}, t}^{\left(\iota^{\prime}\right)} R_{t_{0}, t}^{\left(\iota^{\prime \prime}\right)}
$$

has meaning by virtue of the restrictions imposed on the coefficients of the series in $\stackrel{0}{\mathcal{Q}}_{m}(\lambda)$.

Such a definition of multiplication, together with termwise addition, gives a ring structure in $\stackrel{0}{\mathscr{Q}}_{m}(\lambda)$ and an $\stackrel{0}{\mathscr{Q}}_{m}(\lambda)$-module structure in $\mathbb{Q}_{m}(\lambda)$.

We note that for arbitrary $\left({ }^{2}\right) Q \in \Re$ the element $\lambda-Q$ is invertible in $\stackrel{\mathbb{Q}}{1}_{1}(\lambda)$, and

$$
(\lambda-Q)^{-1}=\sum_{m=0}^{\infty} \lambda^{-m+1} Q^{m}
$$

Let $\mathcal{L}(\lambda)=\Sigma_{\iota \in \mathbf{Z}^{(m)}} Q^{\prime} \lambda^{\iota}$ be a series in $\mathbb{Q}_{m}(\lambda)$. As usual, we call the coefficient $Q^{(-1, \ldots,-1)}$ of $\lambda_{1}^{-1} \cdots \lambda_{m}^{-1}$ the residue of the series $\mathcal{L}(\lambda)$ at zero and denote it by

$$
Q^{(-1, \ldots,-1)}=\operatorname{res} \mathfrak{L}(\lambda)
$$

We consider an arbitrary formal power series $G(\lambda)=\Sigma_{0}^{\infty} G_{\alpha} \lambda_{0}^{\alpha}$ with scalar coefficients. It is clear that such a series belongs, in particular, to an $\mathbb{Q}_{1}(\lambda)$-module of $\stackrel{0}{\mathbb{Q}_{1}}(\lambda)$. In addition, as is not difficult to verify,

$$
\operatorname{res}\left\{G(\lambda)(\lambda-Q)^{-1}\right\}=\sum_{\alpha=0}^{\infty} G_{\alpha} Q^{\alpha}=G(Q)
$$

for any $Q \in \mathbb{Q}$.

Let $F: \stackrel{0}{\mathscr{Q}}_{m}(\lambda) \rightarrow \stackrel{0}{\mathscr{Q}}_{m}(\lambda)$ be a mapping of $\stackrel{0}{\mathscr{Q}}_{m}(\lambda)$ into itself. We call the mapping $\partial_{\mathfrak{Q}} F$ : $\stackrel{0}{\mathbb{Q}}_{m}(\lambda) \rightarrow \stackrel{0}{\mathbb{Q}}_{m}(\lambda)$ defined by

$$
\partial_{\mathfrak{Q}} F(\mathfrak{R}(\lambda))=\left.\frac{d}{d \varepsilon}\right|_{\varepsilon=0} F(\mathfrak{L}(\lambda)+\varepsilon \mathfrak{\varepsilon}(\lambda))
$$

a differential of the mapping $F$ at the "point" $\mathfrak{L}(\lambda) \in \stackrel{0}{\mathbb{Q}}_{m}(\lambda)$ if the derivative on the right-hand side exists for all $\mathfrak{R}(\lambda) \in \stackrel{0}{\mathbb{Q}}_{m}(\lambda)$.

The usual Leibniz rule for the differentiation of a product,

$$
\partial_{\mathfrak{L}}(F G)(\mathfrak{R}(\lambda))=\left(\partial_{\mathfrak{L}} F(\mathfrak{R}(\lambda))\right) G(\mathfrak{R}(\lambda))+F(\mathfrak{L}(\lambda)) \partial_{\mathfrak{L}} G(\mathfrak{R}(\lambda)),
$$

holds for this differential, and (under suitable assumptions) the rule for differentiating a composition,

$$
\partial_{\mathfrak{Q}}(F \circ G)(\mathfrak{N}(\lambda))=\partial_{F(\mathfrak{l})} F \circ \partial_{\mathfrak{Q}} G(\mathfrak{R}(\lambda))
$$

(2) We omit the arguments $t_{0}, t$, and $\vec{X}_{t}$ in the symbol $Q_{t_{0}, t}\left(\vec{X}_{\tau}\right) \in \mathbb{Q}$ for a ring element. 
Let $F$ be such that $\mathfrak{L}(\lambda) \in \stackrel{0}{\mathbb{Q}}_{m}(\lambda)$ exists for all $F(\mathfrak{L}(\lambda))^{-1}$. We denote the mapping $\mathfrak{L}(\lambda) \rightarrow F(\mathfrak{L}(\lambda))^{-1}$ by the symbol $1 / F$ (in contrast to the inverse $F^{-1}$ ). An application of the Leibniz rule to the identity $F(\mathfrak{L}(\lambda)) F(\mathfrak{L}(\lambda))^{-1}=\mathrm{Id}$ gives an expression for the differential of $1 / F$ :

$$
\partial_{\mathfrak{L}}(1 / F)(\mathfrak{R}(\lambda))=-F(\mathfrak{L}(\lambda))^{-1} \mathfrak{R}(\lambda) F(\mathfrak{L}(\lambda))^{-1}
$$

We return to (5.2). We denote the mapping of $Q$ which associates with an arbitrary series $Q_{t_{0}, t}\left(\vec{X}_{\tau}\right) \in \mathbb{Q}$ the series $\varphi\left(\operatorname{ad} Q_{t_{0}, t}\left(\vec{X}_{\tau}\right)\right) \vec{X}_{t}$ by $\Psi_{t}: \mathbb{Q} \rightarrow \mathbb{Q}$. Our equation takes the form

$$
\frac{d}{d t} V_{t}=\Psi_{t}\left(V_{t}\right), \quad V_{t} \in \mathscr{R}
$$

Finally, we denote by $\vec{\Psi}$, the mapping which associates with each differentiable mapping $F$ in the ring $Q \subset \subset \stackrel{0}{\mathbb{Q}}_{m}(\lambda)$ the mapping $Q \rightarrow \partial_{Q} F\left(\Psi_{t}(Q)\right)$ in the same ring. The analogy with ordinary vector fields is obvious.

With the help of $\vec{\Psi}_{t}$, we can write a solution of (5.5) in precisely the same way as in the case of ordinary differential equations. We have

$$
\begin{gathered}
V_{t}=\int_{t_{0}}^{t} \Psi_{\tau_{1}}\left(V_{\tau_{1}}\right) d \tau_{1}=\int_{t_{0}}^{t} \Psi_{\tau_{1}}(0) d \tau_{1}+\int_{t_{0}}^{t} d \tau_{1} \int_{t_{0}}^{\tau_{1}} \partial_{V_{\tau_{2}}} \Psi_{\tau_{1}}\left(\frac{d V_{\tau_{2}}}{d \tau_{2}}\right) d \tau_{2} \\
=\int_{t_{0}}^{t} \Psi_{\tau_{1}}(0) d \tau_{1}+\int_{i_{0}}^{t} d \tau_{1} \int_{t_{0}}^{\tau_{1}} d \tau_{2} \vec{\Psi}_{\tau_{2}} \Psi_{\tau_{1}}\left(V_{\tau_{2}}\right) \\
=\sum_{\alpha=1}^{m-1} \int_{t_{0}}^{t} d \tau_{1} \int_{t_{0}}^{\tau_{1}} d \tau_{2} \ldots \int_{t_{0}}^{\tau_{\alpha-1}} d \tau_{\alpha} \vec{\Psi}_{\tau_{a}} \circ \ldots \circ \vec{\Psi}_{\tau_{2}} \Psi_{\tau_{1}}(0) \\
+\int_{t_{0}}^{t} d \tau_{1} \int_{t_{0}}^{\tau_{1}} d \tau_{2} \ldots \int_{t_{0}}^{\tau_{m-1}} d \tau_{m} \vec{\Psi}_{\tau_{m}} \circ \ldots \circ \vec{\Psi}_{\tau_{2}} \Psi_{\tau_{1}}\left(V_{\tau_{m}}\right) \\
=\left(\sum_{m=1}^{\infty} \int_{t_{0}}^{t} d \tau_{1} \int_{t_{0}}^{\tau_{1}} d \tau_{2} \ldots \int_{t_{0}}^{\tau_{m-1}} d \tau_{m} \vec{\Psi}_{\tau_{m}} \circ \ldots \circ \vec{\Psi}_{\tau_{1}}\right) \operatorname{Id}(0)=\overrightarrow{\exp } \int_{t_{0}}^{t} \vec{\Psi}_{\tau} d \tau \operatorname{Id}(0) .
\end{gathered}
$$

Thus in order to find the chronological series $V_{t_{0} t}\left(\vec{X}_{\tau}\right)$, it remains to calculate the polynomial

$$
\mathfrak{g}_{m}\left(\vec{X}_{1}, \ldots, \vec{X}_{m}\right)=\vec{\Psi}_{1} \circ \ldots \circ \vec{\Psi}_{m-1} \Psi_{m}(0)
$$

The unknown series then takes the form

$$
V_{t_{0}, t}\left(\vec{X}_{\tau}\right)=\sum_{m=1}^{\infty} \int_{t_{0}}^{t} d \tau_{1} \int_{\dot{t}_{0}}^{\tau_{1}} d \tau_{2} \ldots \int_{t_{0}}^{\tau_{m-1}} d \tau_{m} \mathfrak{g}_{m}\left(\vec{X}_{\tau_{1}}, \ldots, \vec{X}_{\tau_{m}}\right)
$$

To carry out the calculation, we make use of (5.3). The mapping $\Psi_{m}$ can be expressed in the form

$$
\Psi_{m}(Q)=\varphi(\operatorname{ad} Q) \vec{X}_{m}=\operatorname{res} \varphi(\lambda)(\lambda-\operatorname{ad} Q)^{-1} \vec{X}_{m}
$$


By virtue of (5.4) and the linearity of the operation of taking the residue, we get

$$
\begin{gathered}
\vec{\Psi}_{2} \Psi_{1}(Q)=\partial_{Q} \Psi_{1}\left(\Psi_{2}(Q)\right)=\operatorname{res} \varphi(\lambda)(\lambda-\operatorname{ad} Q)^{-1} \operatorname{ad} \Psi_{2}(Q)(\lambda-\operatorname{ad} Q)^{-1} \vec{X}_{1} \\
=\operatorname{res} \varphi\left(\lambda_{1}\right) \varphi\left(\lambda_{2}\right)\left(\lambda_{1}-\operatorname{ad} Q\right)^{-1} \operatorname{ad}\left(\left(\lambda_{2}-\operatorname{ad} Q\right)^{-1} \vec{X}_{2}\right)\left(\lambda_{1}-\operatorname{ad} Q\right)^{-1} \vec{X}_{1} .
\end{gathered}
$$

Consequently

$$
\begin{gathered}
\mathfrak{g}_{1}\left(\vec{X}_{1}\right)=\Psi_{1}(0)=\varphi(0) \vec{X}_{1}=\vec{X}_{1}, \\
\mathfrak{g}_{2}\left(\vec{X}_{1}, \vec{X}_{2}\right)=\vec{\Psi}_{2} \Psi_{1}(0)=\operatorname{res} \frac{\varphi\left(\lambda_{1}\right) \varphi\left(\lambda_{2}\right)}{\lambda_{1}^{2} \lambda_{2}} \text { ad } \vec{X}_{2} \vec{X}_{1} \\
=\varphi^{\prime}(0) \varphi(0) \text { ad } \vec{X}_{2} \vec{X}_{1}=\frac{1}{2} \text { ad } \vec{X}_{2} \vec{X}_{1} .
\end{gathered}
$$

If we use (5.4) and Leibniz' rule for differentiating a product, it is not difficult, by means of successive differentiation, to represent $\vec{\Psi}_{m} \circ \ldots \circ \vec{\Psi}_{2} \Psi_{1}(Q)$ in the form of a residue of some series in $\mathcal{Q}_{m}(\lambda)$ for any given $m$, and then, putting $Q=0$ and taking the residue, to find $g_{m}\left(\vec{X}_{1}, \ldots, \vec{X}_{m}\right)$. It is clear from the method of constructing the $g_{m}$ that they satisfy all of the hypotheses of Proposition 5.1.

The procedure for calculating the $\mathfrak{g}_{m}$ can be simplified considerably. We describe one of the possible algorithms.

2. Construction of the polynomials $g_{m}$. Let Ass $\left(\mathrm{ad}, \zeta_{1}, \ldots, \zeta_{m}\right)$ be the free associative algebra over $\mathbf{R}$ with generators ad, $\zeta_{1}, \ldots, \zeta_{m}$. The elements of this algebra are all posssible linear combinations of words made from the alphabet ad, $\zeta_{1}, \ldots, \zeta_{m}$.

We call a word made up of the "letters" ad, $\zeta_{1}, \ldots, \zeta_{m}$, regular if by the introduction of suitable parentheses it can be expressed as a commutator polynomial in $\zeta_{1}, \ldots, \zeta_{m}$ with the usual meaning of the symbol ad,

$$
\left(\operatorname{ad} \zeta_{1}\right) \zeta_{2}=\left[\zeta_{1}, \zeta_{2}\right]=\zeta_{1} \zeta_{2}-\zeta_{2} \zeta_{1}
$$

For example, the words ad $\zeta_{2} \zeta_{1}$, ad ad $\zeta_{3} \zeta_{2} \zeta_{1}=\left[\left[\zeta_{3}, \zeta_{2}\right], \zeta_{1}\right]$ are regular, and the words ad $\zeta_{2}$, ad $\zeta_{2} \zeta_{1} \zeta_{3}$ are not regular. We note that the parentheses in a regular word can be inserted in only one way to get the "commutator monomial."

Let $w$ be some word. With each variable $\zeta_{k}$ occurring in $w$ we associate a natural number called the depth of $\zeta_{k}$ in $w$. The depth is defined in the following way. We represent $w=w_{1} \zeta_{k} w_{2}$, where $w_{1}$ is a (possibly empty) word which does not contain $\zeta_{k}$, and we assume that $w=v_{1} \cdots v_{l}$, where each $v_{j}$ is one of the generators of our algebra.

We define the set $J \subset\{1, \ldots, l\}$ by means of the following rule: $i \in J$ if and only if the following two conditions are satisfied: 1) the number of occurrences of the generator ad in the word $v_{i} v_{i+1} \cdots v_{l}$ is $\frac{1}{2}(l-i+1)$; 2) for each $i^{\prime}>i$, the number of occurrences of ad in $v_{i^{\prime}} v_{i^{\prime}+1} \cdots v_{l}$ does not exceed $\left(l-i^{\prime}+1\right) / 2$. The depth of $\zeta_{k}$ in $w$ is equal (by definition) to the number of elements in $J$.

We mention an equivalent definition of the depth of a variable in a word which motivates the term "depth." In the terminology developed above, the depth of $\zeta_{k}$ in the word $w$ is the number of regular words of the form $v_{i} v_{i+1} \cdots v_{l} \zeta_{k}$, where $i<l$. To prove the equivalence of the two definitions, it suffices to note that the word $v_{i} \cdots v_{l} \zeta_{k}$ is regular if and only if $v_{i} \cdots v_{l}$ satisfies the conditions 1 ) and 2) above.

With each word $w$ we associate a differentiation $\mathscr{D}(w)$ of the algebra Ass $\left(\mathrm{ad}, \zeta_{1}, \ldots, \zeta_{m}\right)$ which acts on the generators according to the rule $\mathfrak{D}(w) \mathrm{ad}=w$ ad, $\mathfrak{D}(w) \zeta_{i}=w \zeta_{i}$, and which is extended to the remaining elements of the algebra by 
linearity and Leibniz' rule. For example,

$$
\mathfrak{D}\left(\zeta_{i}\right) \operatorname{ad} \zeta_{j}=\zeta_{i} \text { ad } \zeta_{j}+\operatorname{ad} \zeta_{i} \zeta_{j}
$$

To construct the polynomials $g_{m}$ we need the sequence $b_{0}=1, b_{\alpha}=B_{\alpha} / \alpha$ !, $\alpha=$ $2,3, \ldots$, of real numbers, where $B_{\alpha}$ are the Bernoulli numbers.

We consider now the element

$$
\mathfrak{D}\left(\operatorname{ad} \zeta_{m}\right) \circ \ldots \circ D\left(\operatorname{ad} \zeta_{2}\right) \zeta_{1} \in \operatorname{Ass}\left(\operatorname{ad}, \zeta_{1}, \ldots, \zeta_{m}\right),
$$

which is obtained from $\zeta_{1}$ by successive applications of the differentiations $\mathfrak{D}\left(\operatorname{ad} \zeta_{2}\right), \ldots, \mathfrak{D}\left(\operatorname{ad} \zeta_{m}\right)$ and which is a sum of $(2 m-3)$ !! regular words:

$$
\mathfrak{D}\left(\operatorname{ad} \zeta_{m}\right) \circ \ldots \circ \mathfrak{D}\left(\operatorname{ad} \zeta_{2}\right) \zeta_{1}=w_{1}+\ldots+w_{(2 m-3) ! !}
$$

We denote the depth of $\zeta_{1}$ in $w_{j}$ by $v_{i j}$, and we put $g_{1}\left(\zeta_{1}\right)=\zeta_{1}$ and

$$
\mathfrak{g}_{m}\left(\zeta_{1}, \ldots, \zeta_{m}\right)=\sum_{\alpha=1}^{(2 m-3) ! !} b_{v_{1 \alpha}} \ldots b_{v_{m \alpha}} w_{\alpha}^{\prime}, \quad m \geqslant 2 .
$$

For example,

$$
\begin{gathered}
\mathfrak{g}_{3}\left(\zeta_{1}, \zeta_{2}, \zeta_{3}\right)=\frac{1}{6} \text { ad } \zeta_{3} \text { ad } \zeta_{2} \zeta_{1}+\frac{1}{4} \text { ad ad } \zeta_{3} \zeta_{2} \zeta_{1}+\frac{1}{6} \text { ad } \zeta_{2} \text { ad } \zeta_{3} \zeta_{1} \\
\left.=\frac{1}{6}\left[\zeta_{3},\left[\zeta_{2}, \zeta_{1}\right]\right]+\frac{1}{4}\left[\mid \zeta_{3}, \zeta_{2}\right], \zeta_{1}\right]+\frac{1}{6}\left[\zeta_{2}, \mid \zeta_{3}, \zeta_{1} \|\right]
\end{gathered}
$$

After simple transformations using the Jacobi identity, we get

$$
\left.\left.\mathfrak{g}_{3}\left(\zeta_{1}, \zeta_{2}, \zeta_{3}\right)=\frac{1}{6}\left(\left[\zeta_{3}, \mid \zeta_{2}, \zeta_{1}\right]\right]+\left[\mid \zeta_{3}, \zeta_{2}\right], \zeta_{1}\right]\right)
$$

Similarly,

$$
\begin{gathered}
g_{4}\left(\zeta_{1}, \zeta_{2}, \zeta_{3}, \zeta_{4}\right) \\
=\frac{1}{12}\left(\left[\left[\zeta_{4}, \zeta_{3}\right], \mid \zeta_{2}, \zeta_{1}\right]\right]+\left[\left[\left[\zeta_{4}, \zeta_{3}\right], \zeta_{2}\right], \zeta_{1}\right]+\left[\zeta_{4},\left[\left[\zeta_{3}, \zeta_{2}\right], \zeta_{1}\right]\right] \\
\left.+\left[\zeta_{3},\left[\left[\zeta_{4}, \zeta_{2}\right], \zeta_{1}\right]\right]\right) .
\end{gathered}
$$

This algorithm for the construction of $g_{m}$ is essentially a formalization of the procedure for calculating the $g_{m}$ by means of residues described earlier, as can be verified by simple induction on $m$.

REMARK. Let $\vec{\varphi}$ be an analytic vector field over the straight line corresponding to the function $\varphi(t)=-t /\left(e^{-t}-1\right)$. Using the fact that

$$
\vec{\Psi}_{1} \circ \ldots \circ \vec{\Psi}_{m-1} \Psi_{m}(0)=\sum_{\alpha=1}^{(2 m-3) ! !} b_{v_{1 \alpha}} \ldots b_{v_{m \alpha}} w_{\alpha},
$$

it is easy to get that

$$
\sum_{\alpha=1}^{(2 m-3) ! !} b_{v_{1 \alpha}} \ldots b_{v_{m \alpha}}=\overrightarrow{\varphi^{m}} E(0)
$$

(here $E$ is the function which gives the identity mapping of the straight line, $E(t) \equiv t$ ). 
Similarly, if

$$
\chi(z)=\sum_{\alpha=0}^{\infty}\left|b_{\alpha}\right| z^{\alpha}=\frac{z}{2}\left(1-\operatorname{ctg} \frac{z}{2}\right)+2
$$

and $\vec{\chi}$ is the vector field corresponding to the function $\chi(t)$, then

$$
\sum_{\alpha=1}^{(2 m-3) ! !}\left|b_{v_{1 \alpha}} \ldots b_{v_{m \alpha}}\right|=\vec{\chi}^{m} E(0)
$$

Using this result (see also (5.7)), we can estimate the constants $C_{1}$ and $C_{2}$ in (4.5). We will, however, not pursue this.

3. Convergence of the series. In general, the series $\vec{V}_{t_{0}, t}\left(\vec{X}_{\tau}\right) E$ diverges, but in certain situations it can converge.

We assume that $B$ is some subalgebra of the Lie algebra $\operatorname{Der}(\Phi)$ and that a norm $\|\cdot\|$, where $\|[\vec{X}, \vec{Y}]\|<\|\vec{X}\|\|\vec{Y}\| \forall \vec{X}, \vec{Y} \in B$, is given on $B$, making it into a Banach Lie algebra.

Proposition 5.2. Let $\vec{X}_{t}$ be a nonstationary field, and let $\vec{X}_{t} \in B$ for all $t \in \mathbf{R}$. If $\int_{t_{0}}^{t}\left\|X_{\tau}\right\| d \tau<0.44$, then $V_{t_{0}, t}\left(\vec{X}_{\tau}\right)$ converges absolutely in $B$.

Proof. Suppose that $\vec{X}_{1}, \ldots, \vec{X}_{m} \in B$. We estimate $\left\|g_{m}\left(\vec{X}_{1}, \ldots, \vec{X}_{m}\right)\right\|$. We note that each variable $\vec{X}_{i}, i=1, \ldots, m$, occurs in the word $w_{j_{\vec{m}}}(j=1,2, \ldots,(2 m-3) ! !$; see (5.6)) precisely once. Consequently $\left\|w_{j}\right\|<\left\|\vec{X}_{1}\right\| \cdots\left\|\vec{X}_{m}\right\|$. Thus

$$
\begin{gathered}
\left\|\mathfrak{g}_{m}\left(\vec{X}_{1}, \ldots, \vec{X}_{m}\right)\right\|=\left\|\sum_{\alpha=1}^{(2 m-3) ! !} b_{v_{1 \alpha}} \ldots b_{v_{m \alpha}} w_{\alpha}\right\| \\
\leqslant\left(\sum_{\alpha=1}^{(2 m-3) \| !}\left|b_{v_{1 \alpha}} \ldots b_{v_{m \alpha}}\right|\right)\left\|\vec{X}_{1}\right\| \ldots\left\|\vec{X}_{m}\right\|=\overrightarrow{\chi \chi \chi}^{m} E(0)\left\|\vec{X}_{1}\right\| \ldots\left\|\vec{X}_{m}\right\| .
\end{gathered}
$$

Therefore the $m$ th term of $V_{t_{0, t}}\left(\vec{X}_{\tau}\right)$ is bounded above by

$$
\overrightarrow{\chi^{m}} E(0) \int_{t_{0}}^{t} d \tau_{1} \int_{t_{0}}^{\tau_{1}} d \tau_{2} \ldots \int_{t_{0}}^{\tau_{m-1}} d \tau_{m}\left\|\vec{X}_{\tau_{1}}\right\| \ldots\left\|\vec{X}_{\tau_{m}}\right\|=\frac{\vec{\chi}^{m} E(0)}{m !}\left(\int_{t_{0}}^{t}\left\|X_{\tau}\right\| d \tau\right)^{m} .
$$

It remains to estimate the radius of convergence of $\Sigma_{0}^{\infty}\left(\vec{\chi}^{m} / m !\right) E(0) \theta^{m}$.

The Maclaurin expansion of $\chi(z)$ has radius of convergence $2 \pi$. Arguing as in $\$ 2.1$, we get that, for any $\sigma \in(0,2 \pi)$,

$$
\vec{\chi}^{m} E(0) \leqslant(m-1) ! \frac{\sigma}{2}\left(\frac{2 M(\sigma)}{\sigma}\right)^{m}
$$

where $M(\sigma)=\max _{z \in C,|z|=\sigma}|\chi(z)|$. Furthermore,

$$
|\chi(z)|=\left|\sum_{\alpha=0}^{\infty}\right| b_{\alpha}\left|z^{\alpha}\right| \leqslant \sum_{\alpha=0}^{\infty}\left|b_{\alpha}\right||z|^{\alpha}=\chi(|z|), \quad|z| \leqslant 2 \pi .
$$

Consequently $M(\sigma)=\chi(\sigma)$. Thus the series in which we are interested converges for $|\theta|<\max _{0<\sigma<2 \pi} \sigma / 2 \chi(\sigma)=0.44 \ldots$ This proves the proposition.

At the same time we get the estimate

$$
\left\|V_{t_{0}, t}\left(\vec{X}_{\tau}\right)\right\| \leqslant e^{\int^{t}\left\|\overrightarrow{X_{\tau}}\right\| d \tau \vec{x}} E(0)
$$


COROLlaRY. Under the hypotheses of the proposition, the flow $\hat{P}_{t_{0, t}}=e^{V_{t_{0}, t}\left(\vec{X}_{r}\right)}$ coincides with $\overrightarrow{\exp } \int_{t_{0}}^{t} \vec{X}_{\tau} d \tau$.

Indeed,

$$
\begin{gathered}
\frac{d}{d t} e^{V_{t_{0}, t}\left(X_{\tau}\right)}=e^{V_{t_{0}, t}\left(\dot{X}_{\tau}\right)} \circ \int_{0}^{1} e^{-\theta \operatorname{ad} V_{t_{0}, t}\left(\vec{X}_{\tau}\right)} d \theta \frac{d}{d t} V_{t_{0}, t}\left(\vec{X}_{\tau}\right) \\
=-e^{V_{t_{0}, t}\left(\vec{X}_{\tau}\right)} \circ \varphi\left(\operatorname{ad} V_{t_{0}, t}\left(\vec{X}_{\tau}\right)\right)^{-1} \circ \varphi\left(\operatorname{ad} V_{t_{0}, t}\left(\vec{X}_{\tau}\right)\right) \vec{X}_{t}=e^{V_{t_{0}, t}\left(\vec{X}_{\tau}\right)} \circ \vec{X}_{t} .
\end{gathered}
$$

\section{§6. Two examples of applications of the formulas}

In subsequent articles we intend to consider applications of the calculus we have developed, mainly to a study of necessary conditions for extremality of high orders. We give here only two simple examples which illustrate possible applications of our results.

1. Control by means of quasistationary fields. In this subsection we characterize in invariant terms those controlled equations which, from the point of view of control theory, must be considered as equivalent to linear systems.

First of all, we introduce a criterion for the commutativity of two nonstationary vector fields of a special form.

Proposition 6.1. Let $\vec{X}, \vec{Y}_{1}$, and $\vec{Y}_{2}$ be (smooth) vector fields. The field $e^{t \text { ad } \vec{x}} \vec{Y}_{1}$ commutes with the field $e^{s \text { ad } \vec{X}_{Y_{2}}}$ for all $t$ and $s$ if and only if

$$
\left[\vec{Y}_{1}, \operatorname{ad}^{j} \vec{X}_{2}\right]=0 \text { for } j=0,1, \ldots, 2 n-1
$$

Proof. The necessity is obvious: it suffices to differentiate the identity [ $\vec{Y}_{1}, e^{t \text { ad } \vec{X}} \vec{Y}_{2}$ ] $=0 j$ times with respect to $t$.

We now establish the sufficiency of (6.1). Because of the identity

$$
\left.\left[e^{t \operatorname{ad} X \vec{Y}_{1}}, e^{s \mathrm{ad} X} \vec{Y}_{2}\right]=e^{t \operatorname{ad} X} \vec{Y}_{1}, e^{(t-s) \operatorname{ad} X} \vec{Y}_{2}\right],
$$

the sufficiency will be established if we prove 1) the commutativity of $\vec{Y}_{1}$ with $e^{\tau \text { ad }} \vec{X}_{Y_{2}}$ for all $\tau$ and 2) that if (6.1) is satisfied then all of the brackets

$$
\left[\operatorname{ad}^{i} X \vec{Y}_{1}, \text { ad } i \vec{X} \vec{Y}_{2}\right], \quad 0 \leqslant i \leqslant n-1,0 \leqslant j \leqslant n,
$$

vanish.

We get 2) at once if we compare the Taylor series expansions of the right and left sides of (6.2).

For each $x \in \mathbf{R}^{n}$, we denote the largest number such that the vectors $\vec{Y}_{1}(x)$, ad $\vec{X} \vec{Y}_{1}(x), \ldots, \operatorname{ad}^{k-1} \vec{X} \vec{Y}_{1}(x)$ are linearly independent by $k(x)$; similarly, $l(x)$ is the largest number such that $\vec{Y}_{2}(x), \ldots$, ad $^{l-1} \vec{X}_{2}(x)$ are linearly independent. It is clear that $k(x)$ and $l(x)$ do not exceed $n$. In addition, $k(x)$ and $l(x)$, which take on finitely many values, are lower semicontinuous and consequently locally constant on some open dense subset of $\mathbf{R}^{\boldsymbol{n}}$. To prove the proposition, it suffices to examine each component of this open set separately. Therefore we may assume that $k(x) \equiv k$ and $l(x) \equiv l$ are constants. In this case,

$$
\operatorname{ad}^{k} \vec{X} \vec{Y}_{1}=\sum_{\alpha=n}^{k-1} a_{\alpha} \operatorname{ad}^{\alpha} \vec{X} \vec{Y}_{1}, \quad \operatorname{ad}^{l} \vec{X} Y_{2}=\sum_{\beta=0}^{l-1} b_{\beta} \operatorname{ad}^{\beta} \vec{X} \vec{Y}_{2},
$$


where $a_{\alpha}$ and $b_{\beta}$ are smooth scalar functions. Suppose that $0<i<n-1$; then, by virtue of 2),

$$
0=\left[\operatorname{ad}^{i} \vec{X} \vec{Y}_{1}, \operatorname{ad}^{l} \vec{X} \vec{Y}_{2}\right]=\sum_{\alpha=0}^{l-1}\left(\left(\operatorname{ad}^{i} \vec{X} \vec{Y}_{1}\right) b_{\beta}\right) \operatorname{ad}^{\beta} \vec{X} \vec{Y}_{2}
$$

Since the fields ad $\vec{X}^{j} \vec{Y}_{1}, 0<\beta<k-1$, are linearly independent, it follows from this that $\left(\operatorname{ad}^{i} \vec{X} \vec{Y}_{1}\right) b_{\beta}=0, \beta=0,1, \ldots, l-1$. Hence we deduce that $\left(e^{t \text { ad } \vec{X}} \vec{Y}_{1}\right) b_{j}=0$ for all $t$ and $j=0,1, \ldots, l-1$.

Indeed,

$$
\begin{aligned}
& \frac{d^{k}}{d t^{k}}\left(e^{t \operatorname{ad} \vec{X} \vec{Y}_{1}}\right) b_{j}=\left(e^{t \operatorname{ad} \vec{X}} \operatorname{ad}^{k} \vec{X} \vec{Y}_{1}\right) b_{j}=\sum_{\alpha=0}^{k-1}\left(e^{t \operatorname{ad} \vec{X}} a_{\alpha} \operatorname{ad} \alpha \vec{X} \vec{Y}_{1}\right) b_{j} \\
& =\sum_{\alpha=0}^{k-1}\left(e^{t \vec{X}_{a}} a_{\alpha}\right) \circ\left(e^{t \operatorname{ad} \vec{X}} \operatorname{ad}^{\alpha} \vec{X} \vec{Y}_{1}\right) b_{j}=\sum_{\alpha=0}^{k-1}\left(e^{t \vec{X}_{a}} a_{\alpha} \circ \frac{d^{\alpha}}{d t^{\alpha}}\left(e^{t \operatorname{ad} \vec{X}} \vec{Y}_{1}\right) b_{j}\right.
\end{aligned}
$$

Thus $\left(e^{t \text { ad } \vec{x}} \vec{Y}_{1}\right) b_{1}$, as a function of $t$, satisfies a linear differential equation of order $k$. In addition, the function and its first $k-1$ derivatives vanish at $t=0$. Consequently $\left(e^{t \text { ad } \vec{X}} \vec{Y}_{1}\right) b_{j} \equiv 0$.

We put $\vec{Z}_{t}=\left[\vec{Y}_{1}, e^{t \text { ad } \vec{X}} \vec{Y}_{2}\right]$. It is required to prove (see 1)) that the family $\vec{Z}_{t}$ consists of zero vector fields.

We have

$$
\begin{gathered}
\frac{d^{l}}{d t^{l}} \vec{Z}_{t}=\left[\vec{Y}_{1}, \sum_{\alpha=0}^{l-1}\left(e^{\left.t \vec{X}_{b_{\alpha}}\right) \circ e^{t} \mathrm{ad} X} \mathrm{ad} \alpha \vec{X} \vec{Y}_{2}\right]\right. \\
=\sum_{\alpha=0}^{l-1}\left(e^{t \vec{X}_{b_{\alpha}}}\right) \circ\left[\vec{Y}_{1}, e^{t \operatorname{ad} \vec{X}} \operatorname{ad}^{\alpha} \vec{X}_{2}\right]+\sum_{\alpha=0}^{l-1}\left(\vec{Y}_{1} \circ e^{t X} b_{\alpha}\right) \circ e^{t \operatorname{ad} \vec{X}} \mathrm{ad}^{\alpha} \vec{X} \vec{Y}_{2} .
\end{gathered}
$$

Since

$$
\vec{Y}_{1} \circ e^{t \vec{X}_{b}} b_{\alpha}=e^{t \vec{X}} \circ\left(e^{-t \operatorname{ad} \vec{X}} \vec{Y}_{1}\right) b_{\alpha}
$$

the second sum on the right-hand side of (6.3) is equal to zero. Consequently

$$
\frac{d^{l}}{d t^{l}} \vec{Z}_{t}=\sum_{\alpha=0}^{l-1}\left(e^{t \vec{x}} b_{\alpha}\right) \frac{d^{\alpha}}{d t^{\alpha}} \vec{Z}_{t}
$$

Since

$$
\left.\frac{d^{j}}{d t^{j}} \vec{Z}_{t}\right|_{t=0}=\left[\vec{Y}_{1}, \text { ad } i \vec{X}_{2}\right]=0
$$

for $j=0, \ldots, l-1$, it follows that $\vec{Z}_{t} \equiv 0$.

We now consider the controlled equation

$$
x=f(x)+G(x) u, \quad u \in \mathbf{R}^{r},
$$

in $\mathbf{R}^{n}$.

The vector-valued and matrix-valued functions $f(x)$ and $G(x)$ are assumed to be smooth and bounded.

In addition, we assume that the vector fields $e^{t \text { ad } \vec{f}} \vec{G} u$ and $e^{s \text { ad } \vec{f}} \vec{G} v$ commute for all $t, s$ and all $u, v \in \mathbf{R}^{n}$. Proposition 6.1 gives an effective method of verifying this assumption. 
We fix a point $x_{0} \in \mathbf{R}^{n}$ (the initial state) and a moment in time $t>0$. The control theory problem for (6.4) consists in the study of the mapping

$$
u(\tau) \mapsto \overline{\exp } \int_{0}^{t}(f+G u(\tau)) d \tau E\left(x_{0}\right),
$$

given on the space $L_{1}^{r}[0, t]$ of $r$-dimensional vector-valued functions which are summable on $[0, t]$, or given on some subset of this space. By virtue of the fact that

$$
\overrightarrow{\exp } \int_{0}^{t}(\vec{f}-\vec{G} u(\tau)) d \tau=\overrightarrow{\exp } \int_{0}^{t} e^{\tau \operatorname{ad} \vec{f}} \overrightarrow{G u}(\tau) d \tau \circ e^{t \vec{f}},
$$

this reduces to the study of the mapping

$$
u(\tau) \rightarrow \overrightarrow{\exp } \int_{0}^{t} e^{\tau \operatorname{ad} \vec{f}} \overrightarrow{G u}(\tau) d \tau E\left(x_{0}\right),
$$

which corresponds to the controlled equation

$$
\dot{x}=e^{\tau \operatorname{ad} \vec{f}} \overrightarrow{G u} E(x), \quad 0 \leqslant \tau \leqslant t,
$$

with initial condition $x(0)=x_{0}$.

We denote by $D$ the corresponding attainable set, i.e. the image of $L_{1}^{r}[0, t]$ under the mapping (6.5),

$$
D=\left\{\overrightarrow{\exp } \int_{0}^{t} e^{\tau \operatorname{ad} \vec{f}} \overrightarrow{G u}(\tau) d \tau \mid u(\tau) \in L_{1}^{r}[0, t]\right\} \subset \mathbf{R}^{n} .
$$

Proposition 6.2. The attainable set $D$ has an intrinsic commutative Lie group structure, and the fields $e^{\tau \text { ad }} \vec{G} \vec{G}, 0 \leqslant \tau \leqslant t, u \in \mathbf{R}^{\tau}$, generate a space of invariant vector fields on this group.

REMARK. We assume that the dimension of the group is $k(k \leqslant n)$. Since any connected $k$-dimensional commutative Lie group can be obtained by factoring $\mathbf{R}^{k}$ by some (perhaps singular) lattice, it follows that the proposition reduces the controlled equation (6.6) to a linear system in $\mathbf{R}^{k}$ modulo a lattice. For example, the problem of hitting a point for (6.6) is equivalent to the problem of hitting the nodes of an appropriate lattice for a linear system in $\mathbf{R}^{k}$.

Proof of THE Proposition. From the vector fields $e^{\tau \text { ad } \vec{f}} \vec{G} u, 0 \leqslant \tau \leqslant t, u \in \mathbf{R}^{r}$, we choose fields $\vec{Y}_{1}, \ldots, \vec{Y}_{k}$ such that the vectors $Y_{1}\left(x_{0}\right), \ldots, Y_{k}\left(x_{0}\right)$ form a basis of the linear hull of the vectors $\left\{e^{\tau \text { ad } \vec{G}} \vec{G} u E\left(x_{0}\right), 0 \leqslant \tau \leqslant t, u \in \mathbf{R}^{r}\right\}$ (we emphasize that $Y_{1}(x), \ldots, Y_{k}(x)$ need not generate the linear hull of the vectors $e^{\tau \operatorname{ad} \vec{f}} \vec{G} u E(x)$ for $\left.x \neq x_{0}\right)$.

We define a mapping $\mathcal{E}: \mathbf{R}^{k} \rightarrow \mathbf{R}^{n}$ by

$$
\mathcal{E}\left(s_{1}, \ldots, s_{k}\right)=e^{\sum_{\alpha=0}^{k} s_{\alpha} \vec{Y}_{\alpha}} E\left(x_{0}\right) .
$$

Since the fields $\vec{Y}_{i}, i=1, \ldots, k$, commute with each other, we get

$$
\frac{\partial}{\partial s_{j}} \mathcal{E}\left(s_{1}, \ldots, s_{k}\right)=e^{\sum_{\alpha=1}^{k} s_{\alpha} \vec{Y}_{\alpha}} Y_{j}\left(x_{0}\right)=Y_{j}\left(\mathcal{E}\left(s_{1}, \ldots, s_{k}\right)\right), \quad j=1, \ldots, k .
$$


The vectors $Y_{1}(\mathcal{E}(s)), \ldots, Y_{k}(\mathcal{E}(s))$ are linearly independent for any $s=\left(s_{1}, \ldots, s_{k}\right) \in$ $\mathbf{R}^{k}$. In fact, if we assume that $\sum_{\alpha=1}^{k} c_{\alpha} Y_{\alpha}(\mathcal{E}(s))=0$, we get for any function $\varphi \in \Phi$

$$
\begin{gathered}
\left(\sum_{\alpha=1}^{k} c_{\alpha} \vec{Y}_{\alpha}\right) \varphi\left(x_{0}\right)=\left(\sum_{\alpha=1}^{k} c_{\alpha} \vec{Y}_{\alpha}\right) \circ e^{\sum_{\alpha=1}^{k} s_{\alpha} \vec{Y}_{\alpha}} \circ e^{-\sum_{\alpha=1}^{k} s_{\alpha} \vec{Y}_{\alpha}} \varphi\left(x_{0}\right) \\
=e^{\sum_{\alpha=1}^{k} s_{\alpha} \vec{Y}_{\alpha}} \circ \sum_{\alpha=1}^{k} c_{\alpha} \vec{Y}_{\alpha} \circ e^{-\sum_{\alpha=1}^{k} s_{\alpha} \vec{Y}_{\alpha}} \varphi\left(x_{0}\right) \\
=\sum_{\alpha=1}^{k} c_{\alpha} \vec{Y}_{\alpha}\left(\begin{array}{c}
-\sum_{\alpha=1}^{k} s_{\alpha} \vec{Y}_{\alpha} \\
\varphi
\end{array}\right) \\
(\delta(s))=0,
\end{gathered}
$$

i.e. $\sum_{1}^{k} c_{\alpha} Y_{\alpha}\left(x_{0}\right)$, which means that $c_{\alpha}=0, \alpha=1, \ldots, k$.

Thus the vectors $\partial \mathcal{E}(s) / \partial s_{j}$ are linearly independent for all $s \in \mathbf{R}^{k}$; consequently the mapping $\mathcal{E}$ induces a smooth $k$-dimensional manifold structure on $\mathcal{E}\left(\mathbf{R}^{k}\right)$.

Furthermore, the correspondence $\left(s_{1}, \ldots, s_{k}\right) \mapsto e^{\Sigma_{1}^{k_{s}} \vec{Y}_{\alpha}}$ defines a transitive operation of the additive group of $\mathbf{R}^{k}$ on the manifold $\mathcal{E}\left(\mathbf{R}^{k}\right)$. Indeed, since the fields $\vec{Y}_{i}$, $i=1, \ldots, k$, commute, we have

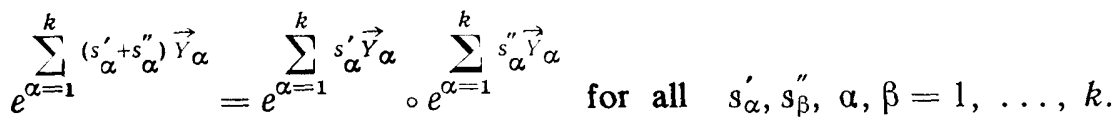

Since $\mathcal{E}\left(\mathbf{R}^{k}\right)$ has dimension $k$, the kernel of this operation is some discrete subgroup $H$ of $\mathbf{R}^{k}$. This means that $\delta\left(\mathbf{R}^{k}\right)$ is isomorphic to $\mathbf{R}^{k} / H$, and the fields $\vec{Y}_{1}, \ldots, \vec{Y}_{k}$ form a basis of the space of invariant vector fields over the group $\mathscr{E}\left(\mathbf{R}^{k}\right)$.

We show that the restrictions of the vector fields $e^{\tau \text { ad } f} \vec{G} u, 0 \leqslant \tau \leqslant t, u \in \mathbf{R}^{r}$, to the set $\mathscr{E}\left(\mathbf{R}^{k}\right)$ are linear combinations (with constant coefficients) of the fields $\vec{Y}_{1}, \ldots, \vec{Y}_{k}$; hence it will follow that $D \subset \mathcal{E}\left(\mathbf{R}^{k}\right)$.

Let $\tau \in[0, t]$ and $u \in \mathbf{R}^{r}$. By virtue of the choice of the $\vec{Y}_{j}, j=1, \ldots, k$, the vector $e^{\tau \text { ad } \vec{f}} \vec{G} u E\left(x_{0}\right)$ can be expressed linearly in terms of $Y_{1}\left(x_{0}\right), \ldots, Y_{k}\left(x_{0}\right)$. We show that a relation of the form

$$
e^{\tau \operatorname{ad} \vec{f}} \overrightarrow{G u} E\left(x_{0}\right)=\sum_{\alpha=1}^{k} c_{\alpha} Y_{\alpha}\left(x_{0}\right)
$$

continues to hold if $x_{0}$ is replaced by any point of $\delta\left(\mathbf{R}^{k}\right)$. We again use the commutativity of the $\vec{Y}_{j}$. For any function $\varphi \in \Phi$ we have

$$
\begin{gathered}
\left(e^{\tau \operatorname{ad} \vec{f}} \overrightarrow{G u} \varphi\right)(\mathscr{E}(s))=e^{\sum_{\alpha=1}^{k} s_{\alpha} \vec{Y}_{\alpha}} \circ e^{\tau \operatorname{ad} \vec{f}} \overrightarrow{G u} \varphi\left(x_{0}\right) \\
=e^{\tau \operatorname{ad} \vec{f}} \overrightarrow{G u}\left(\begin{array}{c}
\sum_{\alpha=1}^{k} s_{\alpha} \vec{r}_{\alpha} \\
e^{\alpha=1}
\end{array}\right)\left(x_{0}\right) \\
=\sum_{\alpha=1}^{k} c_{\alpha} \vec{Y}_{\alpha}\left(\begin{array}{c}
\sum_{\alpha=1}^{k} s_{\alpha} \vec{Y}_{\alpha} \\
e^{\alpha=1}
\end{array}\right)\left(x_{0}\right)=\sum_{\alpha=1}^{k} c_{\alpha} Y_{\alpha}(\mathcal{E}(s)) .
\end{gathered}
$$


To complete the proof, it remains to verify that $\mathcal{E}\left(\mathbf{R}^{k}\right) \subset D$. From Proposition 3.3 we have the identity

$$
\overrightarrow{\exp } \int_{0}^{t} e^{\tau \operatorname{ad} \vec{f}} \overrightarrow{G u}(\tau) d \tau=e^{\int^{\tau} e^{\tau \operatorname{ad} \vec{f} G \vec{u}(\tau)} d \tau}
$$

On the other hand, for each $j$ the restriction of $\vec{Y}_{j}$ to $\mathcal{E}\left(\mathbf{R}^{k}\right)$ can evidently be expressed in the form

$$
\left.\vec{Y}_{j}\right|_{\mathcal{G}\left(\mathbf{R}^{k}\right)}=\int_{0}^{t} e^{\tau \operatorname{ad} \vec{f}} \overrightarrow{G u}(\tau) d \tau
$$

2. The Euler equation for a variational problem. We need certain standard notation and results involving differential forms which we now recall (for details, see [11]).

Any $\Phi$-multilinear skew symmetric mapping

$$
\omega: \operatorname{Der}(\Phi) \times \ldots \times \operatorname{Der}(\Phi) \rightarrow \Phi
$$

is called a differential form of degree $k$ over $\mathbf{R}^{n}$.

The linear space of all such forms is denoted by $\Lambda_{k}^{*}$, and $\Lambda^{*}=\bigoplus_{0}^{n} \Lambda_{k}^{*}$. We note that $\Lambda^{*}$ is an algebra over $\Phi=\Lambda_{0}^{*}$ with respect to the operation of exterior multiplication.

An arbitrary diffeomorphism $P$ induces a mapping $P^{*}: \Lambda^{*} \rightarrow \Lambda^{*}$. In fact, if $\omega \in \Lambda_{k}^{*}$, then

$$
\left(P^{*}(\omega)\left(\vec{X}_{1}, \ldots, \vec{X}_{n}\right)=\hat{P} \omega\left(\operatorname{Ad} \hat{P}^{-1} \vec{X}_{1}, \ldots, \operatorname{Ad} \hat{P}^{-1} \vec{X}_{n}\right)\right.
$$

To each field $\vec{Y} \in \operatorname{Der}(\Phi)$ there corresponds the operator $i_{\vec{Y}}: \Lambda^{*} \rightarrow \Lambda^{*}$ of interior multiplication of differential forms over the field $\vec{Y}$. For any $k \geqslant 0, i_{\vec{Y}}$ acts from $\Lambda_{k}^{*}$ to $\Lambda_{k-1}^{*}$ according to the rule

$$
\left(i_{\vec{Y}} \omega\right)\left(\vec{X}_{1}, \ldots, \vec{X}_{n}\right)=\omega\left(\vec{Y}, \vec{X}_{1}, \ldots, \vec{X}_{n}\right) .
$$

We also define in $\Lambda^{*}$ the coboundary operator (exterior differential) $d$, which acts from $\Lambda_{k}^{*}$ to $\Lambda_{k+1}^{*}$, for each $k \geqslant 0$, according to the rule

$$
\begin{array}{r}
(d \omega)\left(\vec{X}_{1}, \ldots, \vec{X}_{n+1}\right)=\sum_{\alpha=1}^{n+1}(-1)^{\alpha-1} \vec{X}_{\alpha} \omega\left(\vec{X}_{1}, \ldots, \stackrel{\vec{X}}{\alpha}, \ldots, \vec{X}_{n}\right) \\
\quad+\sum_{\alpha<\beta}(-1)^{\alpha+\beta} \omega\left(\left[\vec{X}_{\alpha}, \vec{X}_{\beta}\right], \vec{X}_{1}, \ldots, \overrightarrow{\vec{X}}_{\alpha}, \ldots,{\stackrel{0}{X_{\beta}}}, \ldots, \vec{X}_{n}\right)
\end{array}
$$

(notation of the form $\ldots, \overrightarrow{0}_{\alpha}, \ldots$ means that the field $\vec{X}_{\alpha}$ is deleted from the sequence). The mappings $P^{*}$ and $d$ commute:

$$
P^{*} \circ d=d \circ P^{*}
$$

The operator $L_{\vec{Y}}=i_{\vec{Y}} \circ d+d \circ i_{\vec{Y}}$ is called Lie differentiation along the field $\vec{Y}$. Lie differentiation is actually differentiation in $\Lambda^{*}$, i.e.

$$
L_{\vec{Y}}\left(\omega_{1} \wedge \omega_{2}\right)=\left(L_{\vec{\gamma}} \omega_{1}\right) \wedge \omega_{2}+\omega_{1} \wedge\left(L_{\vec{Y}} \omega_{2}\right) .
$$


The operators $L_{\vec{Y}}$ and $d$ commute, as follows at once from the identity $d \circ d=0$.

We can derive the following useful identity, true for all $\omega \in \Lambda_{1}^{*}$ and $\vec{Y}, \vec{Z} \in \operatorname{Der}(\Phi)$, directly from the definition:

$$
\left(L_{\vec{Y}} \omega\right)(\vec{Z})=\vec{Y} \omega(\vec{Z})-\omega([\vec{Y}, \vec{Z}])
$$

Suppose that the nonstationary field $\vec{X}_{t}$ and the corresponding flow $\hat{P}_{t}=\overrightarrow{\exp } \int_{0}^{t} \vec{X}_{\tau} d \tau$ are given.

Proposition 6.3. The family $P_{t}^{*}$ of mappings of $\Lambda^{*}$ satisfy the equation

$$
\frac{d}{d t} P_{t}^{*}=P_{t}^{*} \circ L_{\vec{X}_{t}}
$$

Proof. Since the operators $L_{\vec{X}}$, and $P_{t}^{*-1} \circ d P_{t}^{*} / d t$ are differentiations in $\Lambda^{*}$, it suffices to show that

$$
\frac{d}{d t} P_{t}^{*}\left(0=P_{t}^{*} \circ L_{\vec{x}_{t}}^{\omega}\right.
$$

for generators of this algebra, i.e. in the case where $\omega$ is a form of degree zero or one. If $\omega$ is of zero degree, i.e. $\omega=\varphi \in \Phi$, then $L_{\vec{X}}, \varphi=X_{t} \varphi$, and the above equality is obvious. Suppose that $\omega \in \Lambda_{1}^{*}$. For any $\vec{Y} \in \operatorname{Der}(\Phi)$ we have (see (6.7) and (6.8))

$$
\begin{gathered}
\frac{d}{d t}\left(P_{t}^{*} \omega\right)(\vec{Y})=\frac{d}{d t} \hat{P}_{t} \omega\left(\operatorname{Ad} \hat{P}_{t}^{-1} \vec{Y}\right) \\
=\hat{P}_{t} \omega\left(-\operatorname{ad} \vec{X}_{t} \operatorname{Ad} \hat{P}_{t}^{-1} \vec{Y}\right)+\hat{P}_{t} \circ \vec{X}_{t} \omega\left(\operatorname{Ad} \hat{P}_{t}^{-1} \vec{Y}\right) \\
=\hat{P}_{t}\left(-\omega\left(\left[\vec{X}_{t}, \operatorname{Ad} \hat{P}_{t}^{-1} \vec{Y}\right]\right)+\vec{X}_{t} \omega\left(\operatorname{Ad} \hat{P}_{t}^{-1} \vec{Y}\right)\right) \\
=\hat{P}_{t} d \omega\left(\vec{X}_{t}, \operatorname{Ad} \hat{P}_{t}^{-1} \vec{Y}\right)+\vec{Y}_{\circ} \circ P_{t} \omega\left(\vec{X}_{t}\right) \\
=\left(P_{t}^{*} \circ i_{\vec{X}_{t}} \circ d \omega\right)(\vec{Y})+\left(d=P_{t}^{*} \circ i_{\vec{X}_{t}} \omega\right)(\vec{Y}) \\
==P_{t}^{*} \circ\left(i_{\vec{X}_{t}} \circ d+d \circ i_{\vec{X}_{t}}\right) \omega(\vec{Y}) .
\end{gathered}
$$

Consequently

$$
\frac{d}{d t} P_{t}^{*} \omega=P_{t}^{*} \circ L_{\vec{X}_{t}} \omega
$$

Thus we may write

$$
\left(\overrightarrow{\exp } \int_{0}^{t} \vec{X}_{\tau} d \tau\right)^{*}=\overrightarrow{\exp } \int_{0}^{t} L_{\vec{X}_{\tau}} d \tau
$$

Let $\mu$ be an $n$ th-degree differential form which vanishes nowhere. Then any other form in $\Lambda_{n}^{*}$ can be obtained by multiplying $\mu$ by some uniquely determined scalar function.

The mapping $\operatorname{div}_{\mu}: \operatorname{Der}(\Phi) \rightarrow \Phi$ is given by $L_{\vec{Y}} \mu=\left(\operatorname{div}_{\mu} \vec{Y}\right) \mu$.

The connection between $\operatorname{div}_{\mu}$ for various $\mu$ is given by the relation

$$
\varphi \operatorname{div}_{\varphi \mu} \vec{Y}=\operatorname{div}_{\mu}(\varphi \vec{Y})=\vec{Y}_{\varphi}+\varphi \operatorname{div}_{\mu} \vec{Y},
$$

which holds for any $\varphi \in \Phi$ and $\vec{Y} \in \operatorname{Der}(\Phi)$. 
We denote the set of all diffeomorphisms which preserve $\mu$ by $\mathscr{P}_{\mu}$, i.e. $P \in \mathscr{P} \Leftrightarrow P^{*} \mu$ $=\mu$. From (6.10) it follows that $\overrightarrow{\exp } \int_{0}^{t} \vec{X}_{\tau} d \tau \in \mathcal{P}_{\mu}$ for an arbitrary nonstationary field $\vec{X}_{t}$ and for all $t$ if and only if $\operatorname{div}_{\mu} \vec{X}_{t} \equiv 0$.

Let $f(x, v), x, v \in \mathbf{R}^{n}$, be a smooth scalar function. To each vector field $\vec{Y}$ there corresponds a function $f(\vec{Y}) \in \Phi$ defined by the relation $f(\vec{Y})(x)=f(x, Y(x))$. We denote by $d_{v} f(\vec{X})$ the first-degree differential form given by

$$
d_{v} f(\vec{X})(\vec{Y})=\left.\frac{d}{d \varepsilon}\right|_{\varepsilon=0} f(\vec{X}+\varepsilon \vec{Y}) .
$$

We fix a bounded region $\Omega_{0} \subset \mathbf{R}^{n}$ with a smooth boundary $\partial \Omega_{0}$, and we define the functional $J$ on the set of flows $P_{0, t}$ which preserve $\mu\left(P_{0, t} \in \mathscr{P}_{\mu}\right.$ for all $\left.t \in \mathbf{R}\right)$ in the following way:

$$
J\left(P_{0, \tau}\right)=\int_{0}^{1} d t \int_{\Omega_{0}} \hat{P}_{0, t} f\left(\overrightarrow{\log } \hat{P}_{0, t}\right) \mu
$$

The $n$ th-degree form under the inside integral sign in (6.12) is obtained by applying the diffeomorphism $\hat{P}_{0, t}$ to the function $f\left(\overrightarrow{l o g} \hat{P}_{0, t}\right)$ and multiplying the result by $\mu$.

We find differential equations which are satisfied by the extremals of this functional for two types of boundary conditions:

1) fixed diffeomorphism $P_{0,1}$;

2) fixed region $\Omega_{1}=P_{0,1}\left(\Omega_{0}\right)$

(condition 1) is essentially more restrictive).

We calculate the first variation of $J$ at the "point" $P_{0,1}$. We will denote the flow $P_{0,1}$ simply by $P_{t}$. In addition, we denote the result of applying an arbitrary first-degree form $\omega$ to some field $\vec{Y}$ by means of angular brackets (as a scalar product) $\omega(\vec{Y})=\langle\omega, \vec{Y}\rangle$.

Let $\vec{X}_{t}=\overrightarrow{\log } \hat{P}_{t}, \operatorname{div}_{\mu} \vec{X}_{t}=0 \forall t \in \mathbf{R}$. Using (3.16) and (2.20), we get

$$
\begin{aligned}
& J\left(\overrightarrow{\exp } \int_{0}^{t}\left(\vec{X}_{\tau}+\vec{Y}_{\tau}\right) d \tau\right)=\int_{0}^{1} d t \int_{\Omega_{0}} \overrightarrow{\exp } \int_{0}^{t}\left(\vec{X}_{\tau}+\vec{Y}_{\tau}\right) d \tau f\left(\vec{X}_{t}+\vec{Y}_{t}\right) \mu \\
= & J\left(P_{t}\right)+\int_{0}^{1} d t \int_{\dot{\Omega}_{0}} \hat{P}_{t}\left\{\left\langle d_{v} f\left(\vec{X}_{t}\right), \vec{Y}_{t}\right\rangle+\int_{0}^{t}\left(\overleftarrow{\exp } \int_{\tau}^{t}-\operatorname{ad} \vec{X}_{\theta} d \theta \vec{Y}_{\tau}\right) d \tau f\left(\vec{X}_{t}\right)\right\} \mu+r,
\end{aligned}
$$

where $\|r\|_{s, K}=O\left(\int_{0}^{1} d t\left(\int_{0}^{t}\left\|Y_{\tau}\right\|_{s+1} d \tau\right)^{2}\right)$ for $s=0,1,2, \ldots$ and any compact set $K$.

The nonstationary vector field

$$
\vec{Z}_{t}=\int_{0}^{t} \overleftarrow{\exp } \int_{\tau}^{t}-\operatorname{ad} \vec{X}_{\theta} d \theta \vec{Y}_{\tau} d \tau
$$

satisfies

$$
\frac{d}{d t} \vec{Z}_{t}=- \text { ad } \vec{X}_{t} \vec{Z}_{t}+\vec{Y}_{t}, \quad \vec{Z}_{0}=0
$$

The first variation $\delta J$ of $J$ at the point $P_{t}$ can be expressed in the following form:

$$
\delta J\left(\vec{Y}_{\tau}\right)=\int_{0}^{1} d t \int_{\Omega_{0}} \tilde{P}_{t}\left\{\left\langle d_{v} f\left(\vec{X}_{t}\right), \vec{Y}_{t}\right\rangle+\vec{Z}_{t} f\left(\vec{X}_{t}\right)\right\} \mu .
$$


Differentiation of the boundary conditions with respect to $\vec{Y}_{t}$ gives $\vec{Z}_{1}=0$ in case 1 ), and $\vec{Z}_{1}$ is tangent to $\partial \Omega_{1}$ in case 2 ).

From this point on, we assume that $\vec{X}_{t}$ depends absolutely continuously on $t$. We transform the expression for $\delta J\left(\vec{Y}_{\tau}\right)$. Using (6.13) and integrating by parts, we get

$$
\begin{gathered}
\int_{0}^{1} d t \int_{\Omega_{0}} \hat{P}_{t}\left\langle d_{v} f\left(\vec{X}_{t}\right), \vec{Y}_{t}\right\rangle \mu=\int_{0}^{1} d t \int_{\Omega_{0}} \hat{P}_{t}\left\langle d_{v} f\left(\vec{X}_{t}\right), \frac{d}{d t} \vec{Z}_{t}+\left[\vec{X}_{t}, \vec{Z}_{t}\right]\right\rangle \\
=\int_{\Omega_{0}} \hat{P}_{1}\left\langle d_{v} f\left(\vec{X}_{1}\right), \vec{Z}_{1}\right\rangle \mu-\int_{0}^{1} d t \int_{\Omega_{0}} \hat{P}_{t}\left\{\vec{X}_{t}\left\langle d_{v} f\left(\vec{X}_{t}\right), \vec{Z}_{t}\right\rangle\right. \\
+\left\langle\frac{d}{d t} d_{v} f\left(\vec{X}_{t}\right), \vec{Z}_{t}\right\rangle-\left\langle d_{v} f\left(\vec{X}_{t}\right),\left[\vec{X}_{t}, \vec{Z}_{t}\right]\right\} \mu .
\end{gathered}
$$

Applying (6.9) to the form $d_{v} f\left(\vec{X}_{t}\right)$ and the fields $\vec{X}_{t}$ and $\vec{Z}_{t}$, we get

$$
\begin{aligned}
& \int_{0}^{1} d t \int_{\dot{\Omega}_{0}} \hat{P}_{t}\left\langle d_{v} f\left(\vec{X}_{t}\right), \vec{Y}_{t}\right\rangle \mu=\int_{\dot{\Omega}_{0}} \hat{P}_{1}\left\langle d_{v} f\left(\vec{X}_{1}\right), \vec{Z}_{1}\right\rangle \mu \\
& \quad-\int_{0}^{1} d t \int_{\Omega_{0}} \hat{P}_{t}\left\langle\frac{d}{d t} d_{v} f\left(\vec{X}_{t}\right)+L_{\vec{X}_{t}} d_{v} f\left(\vec{X}_{t}\right), \vec{Z}_{t}\right\rangle \mu .
\end{aligned}
$$

If we change the variables of integration and take into account the fact that $P_{t}^{*} \mu=\mu$, we arrive at the relation

$$
\begin{gathered}
\delta J\left(\vec{Y}_{\tau}\right)=\int_{\Omega_{1}}\left\langle d_{v} f\left(\vec{X}_{1}\right), \vec{Z}_{1}\right\rangle \mu \\
-\int_{0}^{1} d t \int_{\Omega_{t}}\left\langle\frac{d}{d t} d_{v} f\left(\vec{X}_{t}\right): L_{X_{t}} d_{v} f\left(\vec{X}_{t}\right)-d f\left(\vec{X}_{t}\right), \vec{Z}_{t}\right\rangle \mu .
\end{gathered}
$$

We now suppose that $P_{t}$ is an extremal of $J$. Then $\delta J\left(\vec{Y}_{\tau}\right)=0$ for all admissible $\vec{Y}_{t}$. Consequently

$$
\begin{gathered}
\int_{\Omega_{t}}\left\langle\frac{d}{d t} d_{v} f\left(\vec{X}_{t}\right)+L_{\vec{X}_{t}} d_{v} f\left(\vec{X}_{t}\right)-d f\left(\vec{X}_{t}\right), \vec{Z}\right\rangle \mu=0, \\
\forall t \in[0,1], \forall \vec{Z}, \quad \text { so that } \operatorname{div}_{\mu} \vec{Z}=0 .
\end{gathered}
$$

LEMMA 1. Assume that the first-degree form $\omega$ in the bounded region $\Omega$ with smooth boundary $\partial \Omega$ satisfies

$$
\int_{\mathbf{\Omega}}\langle\omega, \vec{Z}\rangle \mu=0 \quad \forall \vec{Z}, \quad \text { so that } \operatorname{div}_{\mu} \vec{Z}=0 \text {. }
$$

Then $\omega=d \varphi$ for some function $\varphi$ which vanishes on $\partial \Omega$.

Proof. We show first of all that (6.15) does not depend on the choice of the $n$ th-degree form $\mu$ which vanishes nowhere. In fact, any other $n$ th-degree form has the form $\varphi \mu$, where $\varphi \in \Phi$. In addition it follows from (6.11) that $\operatorname{div}_{\mu}(\varphi \vec{Z})=0 \Leftrightarrow \operatorname{div}_{\varphi \mu} \vec{Z}=$ 0 for every function $\varphi$ in $\Phi$ which vanishes nowhere. 
Thus it suffices to prove the lemma for the case where $\mu$ is a volume element in $\mathbf{R}^{n}$, $\mu=\bigwedge_{1}^{n} d x^{i}$. In what follows, we will denote the Laplace operator by $\Delta$,

$$
\Delta=\operatorname{div}_{\mu} \circ \operatorname{grad}=\sum_{\alpha=1}^{n} \frac{\partial^{2}}{\left(\partial x^{\alpha}\right)^{2}}
$$

for any vector fields $\vec{X}$ and $\vec{Y}$ the function $(\vec{X}, \vec{Y})$ in $\Phi$ is given by

$$
(\vec{X}, \vec{Y})(x)=(X(x), Y(x))=\sum_{\alpha=1}^{n} X^{\alpha}(x) Y^{\alpha}(x),|\vec{X}|^{2}=(\vec{X}, \vec{X})
$$

$\omega^{T}$ is the vector field dual to $\omega$ defined by

$$
\left(\omega^{T}, \vec{X}\right)=\langle\omega, \vec{X}\rangle \quad \forall \vec{X} \in \operatorname{Der}(\Phi) .
$$

The function $\varphi$ whose existence is asserted in the lemma is uniquely determined by the conditions

$$
\begin{gathered}
\Delta \varphi=\operatorname{div}_{\mu} \omega^{T} \text { in } \Omega, \\
\varphi=0 \text { on } \partial \Omega .
\end{gathered}
$$

The validity of (6.15) for $d \varphi$ follows easily from the Gauss-Ostrogradskin formula. In fact, if $\operatorname{div}_{\mu} \vec{Z}=0$, then $\langle d \varphi, \vec{Z}\rangle=\vec{Z} \varphi=\operatorname{div}_{\mu}(\varphi \vec{Z})$, and consequently

$$
\int_{\Omega}\langle d \varphi, \vec{Z}\rangle \mu=\int_{\Omega} \operatorname{div}_{\mu}(\varphi \vec{Z}) \mu=\int_{\partial \Omega} \varphi \vec{Z} d n=0 .
$$

On the other hand, $\operatorname{div}_{\mu}\left(\omega^{T}-\operatorname{grad} \varphi\right)=0$. Thus

$$
0=\int_{\dot{\Omega}}\left\langle\omega-d \varphi, \omega^{T}-\operatorname{grad} \varphi\right\rangle \mu=\int_{\Omega}\left|\omega^{T}-\operatorname{grad} \varphi\right|^{2} \mu .
$$

Therefore $\omega=d \varphi$.

Lemma 1 and (6.14) guarantee the existence, for any $t \in[0,1]$, of a function $\varphi_{t}$ such that

$$
\begin{gathered}
\frac{d}{d t} d_{v} f\left(\vec{X}_{t}\right)+L_{\vec{X}_{t}} d_{v} f\left(\vec{X}_{t}\right)=d\left(f\left(\vec{X}_{t}\right)+\varphi_{t}\right) \text { in } \Omega_{t} \\
\varphi_{t}=0 \text { on } \partial \Omega_{t}, \quad \operatorname{div}_{\mu} \vec{X}_{t}=0 .
\end{gathered}
$$

These are equations for extremals of $J$ in the case of boundary condition 1). In case 2), both (6.14) and the fact that

$$
\int_{\Omega_{1}}\left\langle d_{v} f\left(\vec{X}_{1}\right), \vec{Z}\right\rangle \mu=0 \quad \forall \vec{Z} \in \operatorname{Der}(\Phi)
$$

follow from $\delta J=0$, so that $\operatorname{div}_{\mu} \vec{Z}=0$, and $\vec{Z}$ is tangent to $\partial \Omega_{1}$.

LEMMA 2. Assume that the first-degree form $\omega$ in the bounded region $\Omega$ with smooth boundary $\partial \Omega$ satisfies

$$
\int_{\Omega}\langle\omega, \vec{Z}\rangle \mu=0 \quad \forall \vec{Z},
$$

where $\operatorname{div}_{\mu} \vec{Z}=0$ and $\vec{Z}$ is tangent to $\partial \Omega$. Then $\omega=d \varphi$ for some function $\varphi$. 
The proof is similar to that of Lemma 1. In fact, it suffices to consider the case where $\mu$ is a volume element in $\mathbf{R}^{n}$. In this case $\varphi$ is determined by the conditions

$$
\begin{aligned}
& \Delta \varphi=\operatorname{div} \omega^{T} \text { in } \Omega, \\
& \frac{\partial \varphi}{\partial n}=\langle\omega, n\rangle \text { on } \partial \Omega
\end{aligned}
$$

(here $n$ is the exterior unit normal to $\partial \Omega$, and the remaining notation is as in the proof of Lemma 1).

From (6.17) and Lemma 2 it follows that $d_{v} f\left(\vec{X}_{1}\right)=d S_{1}$ in $\Omega_{1}$ for some function $S_{1}$.

We put $\omega_{t}=d_{v} f\left(\vec{X}_{t}\right)$. Then $\omega_{1}=d S_{1}$ and (see (6.16))

$$
\frac{d}{d t} \omega_{t}+L_{\vec{X}_{t}} \omega_{t}=d\left(f\left(\vec{X}_{t}\right)+\varphi_{t}\right) \text { in } \Omega_{t}
$$

The family of forms $\omega_{t}$ is a unique solution of (6.18), since the corresponding homogeneous equation has only one solution (see (6.10) and Proposition 1.1). Making use of the Cauchy formula for the solution of linear differential equations (its validity in this situation can be verified by direct differentiation) and the commutativity of Lie differentiation and exterior differentiation, we get

$$
\begin{aligned}
& \omega_{t}=\overleftarrow{\exp } \int_{1}^{t}-L_{\vec{X}_{\tau}} d \tau d S_{1}+\int_{i}^{t}\left\{\left(\overleftarrow{\exp } \int_{\tau}^{t}-L_{\vec{X}_{\theta}} d \theta\right) d\left(f\left(\vec{X}_{\tau}\right)+\varphi_{\tau}\right)\right\} d \tau \\
& =d\left(\overleftarrow{\exp } \int_{i}^{t}-\vec{X}_{\tau} d \tau S_{1}+\int_{i}^{t}\left(\overleftarrow{\exp } \int_{\tau}^{t}-\vec{X}_{\theta} d \theta\left(f\left(\vec{X}_{\tau}\right)+\varphi_{\tau}\right)\right) d \tau\right) \text {. }
\end{aligned}
$$

Thus $\omega_{t}=d S_{t}$ for some function $S_{t}$ and for all $t$ in $[0,1]$. After removal of the exterior differential, (6.18) takes the form

$$
\frac{d}{d t} S_{t}+\vec{X}_{t} S_{t}=f\left(\vec{X}_{t}\right)+\varphi_{t}
$$

Since $\varphi_{t}=0$ on $\partial \Omega_{t}$, the complete system of equations for the extremals of $J$ in the case of the boundary conditions 2 ) is

$$
\begin{gathered}
\frac{d}{d t} S_{t}+\vec{X}_{t} S_{t}=f\left(\vec{X}_{t}\right) \text { on } \partial_{\Omega} t \\
d S_{t}=d_{v} f\left(\vec{X}_{t}\right) \text { and } \operatorname{div}_{\mu} \vec{X}_{t}=0 \text { in } \Omega_{t}
\end{gathered}
$$

REMARK. In the case where $n=3, \mu=\bigwedge_{1}^{3} d x^{i}$ and $f$ has the form $f(x, v)=\frac{1}{2} \rho|v|^{2}-$ $U(x)$, the variational problem for $J$ with boundary conditions of type 1 ) embodies the principle of least action for the motion of an ideal fluid in a force field with potential $U(x)$, and (6.16) is the corresponding Euler equation (see [10]). The function $\varphi_{t}$ plays the role of the pressure, and the boundary is free. 


\section{BIBLIOGRAPHY}

1. Henry J. Kelley, Richard E. Kopp and H. Gardner Moyer, Singular extremals, Topics in Optimization, Academic Press, New York, 1967, pp. 63-101.

2. R. Gabasov and F. M. Kirillova, The qualitative theory of optimal processes, "Nauka", Moscow, 1971; English transl., Marcel Dekker, New York, 1976.

3. Arthur J. Krener, The high order maximal principle and its application to singular extremals, SIAM J. Control and Optimization 15 (1977), 256-293.

4. Henry Hermes, Local controllability and sufficient conditions in singular problems. I, II, J. Differential Equations 20 (1976), 213-232; SIAM J. Control and Optimization 14 (1976), 1049-1062.

5. H. W. Knobloch, High order necessary conditions and local controllability, Preprint, Math. Inst. Univ. Wurzburg, 1978.

6. A. A. Agračev and R. V. Gamkrelidze, A second order optimality principle for a time-optimal problem, Mat. Sb. 100 (142) (1976), 610-643; English transl. in Math. USSR Sb. 29 (1976).

7. A. A. Agracev, $A$ second-order necessary condition for optimality in the general nonlinear case, Mat. Sb. 102 (144) (1977), 551-568; English transl. in Math. USSR Sb. 31 (1977).

8. R. V. Gamkrelidze, Exponential representation of solutions of ordinary differential equations, Proc. "Equadiff IV" (Fourth Czechoslovak Conf. Differential Equations and Their Applications), Lecture Notes in Math., vol. 703, Springer-Verlag, Berlin and New York,1979, pp. 118-129.

9. M. V. Karasev and M. V. Mosolova, Infinite products and T-products of exponentials, Teoret. i Mat. Fiz. 28 (1976), 189-200; English transl. in Theoret. Math. Phys. 28 (1976).

10. V. I. Arnol'd, Mathematical methods in classical mechanics, "Nauka", Moscow, 1974; English transl., Springer-Verlag, Berlin and New York, 1978.

11. Claude Godbillon, Géométrie différentielle et mécanique analytique, Hermann, Paris, 1969.

Translated by H. T. JONES 